\title{
Le Cheval du gisement Pléistocène moyen de La Micoque (Les Eyzies-de-Tayac, Dordogne) : Equus mosbachensis micoquii nov. ssp.
}

The Horse from the Middle Pleistocene archaeological site of "La Micoque" (Les Eyzies-de-Tayac, Dordogne): Equus mosbachensis micoquii nov. ssp.

\section{Anne Langlois}

\section{OpenEdition}

Journals

Édition électronique

URL : http://journals.openedition.org/paleo/859

DOI : 10.4000/paleo.859

ISSN : 2101-0420

Éditeur

SAMRA

Édition imprimée

Date de publication : 1 décembre 2005

Pagination : $73-110$

ISSN : 1145-3370

Référence électronique

Anne Langlois, «Le Cheval du gisement Pléistocène moyen de La Micoque (Les Eyzies-de-Tayac, Dordogne) : Equus mosbachensis micoquii nov. ssp. », PALEO [En ligne], 17 | 2005, mis en ligne le 23 avril 2010, consulté le 07 juillet 2020. URL : http://journals.openedition.org/paleo/859 ; DOI : https:// doi.org/10.4000/paleo.859

Ce document a été généré automatiquement le 7 juillet 2020

PALEO est mis à disposition selon les termes de la licence Creative Commons Attribution - Pas d'Utilisation Commerciale - Pas de Modification 4.0 International. 


\section{Le Cheval du gisement Pléistocène moyen de La Micoque (Les Eyzies- de-Tayac, Dordogne) : Equus mosbachensis micoquii nov. ssp.}

The Horse from the Middle Pleistocene archaeological site of "La Micoque" (Les Eyzies-de-Tayac, Dordogne): Equus mosbachensis micoquii nov. ssp.

\section{Anne Langlois}

Je voudrais tout d'abord remercier F. Delpech et J.-L. Guadelli pour m'avoir dirigée dans le cadre de ma thèse et ainsi m'avoir permis de mener à bien cette recherche. Je remercie également le Conservateur en chef du Musée National des Eyzies, Jean-Jacques Cleyet-Merle, pour m'avoir confié, pour étude, le matériel du site de La Micoque ainsi que A. Debénath et J.-P. Rigaud qui ont exhuméles vestiges étudiés. Merci également à toutes les personnes qui m'ont aidée au cours de mes années de thèse. Mes derniers remerciements vont aux trois personnes du comité de lecture qui ont relu et corrigé cet article, M.-F. Bonifay, F. Delpech et P. Fernandez.

\section{1 - Problématique}

Dès les premières études paléontologiques, le Cheval de La Micoque fut déterminé comme appartenant à « une grande race d'Equus caballus » (Hauser 1907, p.11). Il serait proche « des lourds chevaux de la période interglaciaire des steppes en Allemagne et se rattache aux dépôts plus anciens de la grotte de Lunel-Viel» (Hauser 1907, p.12). Lors des études récentes de la faune, le Cheval de La Micoque a été attribué à une forme proche d'Equus mosbachensis, notamment par la robustesse de ses molaires. F. Prat précise que ces dents «présentent (...) quelques dispositions archaïques» (Prat 1968, p. 249). Toutefois, le degré d'évolution de ce Cheval au sein du groupe Equus mosbachensis restait à définir. Outre la meilleure connaissance de ce taxon, ce travail devait 
permettre, grâce à une étude biostratigraphique, d'ancrer le gisement de La Micoque dans la chronologie du Pléistocène moyen.

\section{Rappel de systématique : qu'est-ce que l'espèce Equus mosbachensis?}

2 Ce que l'on a coutume d'appeler dans la littérature « chevaux » rassemble les caballins et les sténoniens. Au sein des caballins, différentes formes ont été distinguées. A l'origine, elles avaient été décrites comme des sous-espèces d'Equus caballus (Prat 1968; Guadelli 1987) comme par exemple en France Equus caballus mosbachensis, Equus caballus piveteaui, Equus caballus germanicus, Equus caballus gallicus puis comme des espèces distinctes (Guérin 1996) notamment Equus mosbachensis. Adhérer à l'un ou à l'autre de ces points de vue est délicat. Actuellement, la variabilité spécifique comme la variabilité sous-spécifique est encore incomplètement documentée chez les chevaux fossiles (Guadelli 1987). Comme M.-F. Bonifay (1980), V. Eisenmann et al. (1985) et J.-L. Guadelli et F. Prat (1995), nous pensons, que certaines formes ont droit au rang spécifique et plus particulièrement le groupe des chevaux de type mosbachensis qui s'oppose au groupe caballus plus récent par de nombreux caractères (Langlois 2004).

Equus mosbachensis apparaît au début du Pléistocène moyen, c'est le premier caballin (sensu lato) européen. Il fut découvert dans le gisement de Mosbach en Allemagne, et décrit par W. von Reichenau en 1915 qui le nomme E. caballus mosbachensis car il possédait des caractères morphologiques dentaires propres à l'espèce E. caballus. Ses dimensions crâniennes sont très grandes et son museau long (Eisenmann et al. 1985). Sa taille au garrot a été estimée à environ 1,70 m (Gromova 1949a). Il garde, cependant, des particularités sténoniennes à savoir un front relativement étroit, des dents jugales petites et les os des extrémités assez étroits (Gromova 1949a et 1949b). Les restes ayant servi à sa diagnose proviennent de niveaux archéologiques qui correspondraient à une période tempérée, datant d'un peu moins de 700000 ans (Brüning 1974, 1978). L'extension géographique de ce «Cheval » de Mosbach couvrait l'ensemble de l'Europe, de la France à la Russie (Prat 1968).

4 La quantité de gisements ayant livré des restes attribuables à Equus mosbachensis ainsi que leur présence sur une période relativement longue (Pléistocène moyen) ont favorisé la création de nombreux taxons :

Equus caballus mosbachensis tautavelensis Crégut, 1980 : ce Cheval a été découvert à la Caune de l'Arago (Tautavel, Pyrénées Orientales). Ces restes sont issus des couches archéologiques du sol G, dont l'âge a été longuement discuté (M.A. et H. de Lumley 1971 ; Crégut 1979 ; Eisenmann et al. 1985). Récemment, des datations radiométriques montrent que les niveaux les plus anciens de ce gisement se seraient formés pendant ou avant le stade isotopique 9 , soit vers 300 - $350 \mathrm{ka}$ (Falguères et al. 2004). Nous savons également que ces restes sont associés à une faune de climat froid (Bellai 1995). La morphologie dentaire et certaines dimensions de cet Équidé sont celles d'une forme assez évoluée d'Equus mosbachensis. Toutefois, E. Crégut a noté des caractères propres à ce Cheval comme par exemple les "proportions des phalanges antérieures [qui] sont bien différentes de celles d'Equus mosbachensis, comme d'ailleurs de celles des autres Equidae européens connus jusqu'à ce jour » (Crégut 1980, p.124). En 1985, V. Eisenmann et al considèrent que le Cheval de Tautavel est différent d'Equus mosbachensis; d'après 
ces auteurs, ce Cheval possède des caractères qui le rapprocheraient de l'espèce Equus chosaricus.

Equus mosbachensis palustris Bonifay, 1980: défini à Lunel-Viel (Hérault), dans les ensembles supérieur et inférieur, cet Équidé est représenté par un nombre important de restes. La présence simultanée d'espèces archaïques et évoluées au sein des niveaux archéologiques suggère, pour le gisement, un âge proche de la fin du Pléistocène moyen, autour de 350 ka (Fosse 1994). Cet Équidé a pour caractéristique un crâne de taille moyenne avec une gouttière nasale visible, ce qui est un caractère ancien. Ses dents possèdent des caractères caballins : les molaires supérieures présentent un pli caballin fréquent mais persistent des caractères ancestraux comme, par exemple, « un protocône assez allongé (...) et la brièveté des dernières molaires par rapport à la longueur des molaires » (Bonifay 1980, p.261). La dentition est attribuable à un caballin (sensu lato). Son crâne est toutefois plus petit que celui du Cheval de Mosbach et encore plus que celui trouvé à l'Arago (Eisenmann et al. 1985). Pour M.-F. Bonifay (1980), le Cheval de Lunel-Viel se rapproche de la forme mosbachensis mais il possède des caractères spécifiques qui peuvent être interprétés comme une adaptation particulière à un milieu tempéré et à une zone humide et marécageuse. M.-F. Bonifay (1980) met en parallèle la Camargue actuelle et la zone de Lunel-Viel au cours de l'interglaciaire Mindel-Riss. Elle justifie ainsi le terme de «palustris » choisi pour nommer la nouvelle sous-espèce. Cependant, les reconstitutions du crâne faites par V. Eisenmann et al. (1985) montreraient des ressemblances morphologiques et biométriques entre ce crâne et ceux du Cheval de Przewalski actuel et serait donc à rapprocher de l'espèce Equus przewalskii. Il faut toutefois rappeler que les vestiges crâniens sur lesquels sont fondées les diagnoses ne représentent que quelques crânes fragmentaires dont un seul est complet.

Les différences entre Equus mosbachensis et les deux formes du Sud de la France précédemment citées, portent essentiellement sur la longueur des museaux, il faut savoir que ce caractère n'est pas diagnosique de l'espèce (Eisenmann et al. 1985 p.163 $-164)$.

5 Equus mosbachensis campdepeyri Guadelli et Prat, 1995 : cette nouvelle sous-espèce fut décrite en 1976, dans le site de Camp-de-Peyre (Sauveterre-la-Lémance, Lot-etGaronne). Ces restes sont associés à une faune qui vivait dans un milieu ouvert, sous un climat froid lors d'une période antérieure à l'interglaciaire Mindel / Riss (Delpech et al. 1978). Les fouilles ont fourni des os de chevaux pour la plupart entiers et une tête osseuse endommagée (calvarium, prémaxillaire avec les séries incisivales et la canine gauche, mandibule sans les régions angulaires). Après étude, J.-L. Guadelli et F. Prat (1995) indiquent que ces fragments de crâne ont des dimensions un peu plus faibles que celles du crâne retrouvé à Mosbach mais un peu plus grandes que celui de Lunel-Viel. Par rapport à ce dernier, le museau est plus court et plus large, il s'agit toutefois d'un individu assez jeune (5 ans). L'examen des restes dentaires et osseux montre qu'il s'agit d'un caballin au sens large, dont la hauteur au garrot est de 1,50 m minimum. Ce Cheval est différent des Équidés datant du Pléistocène moyen par sa taille. Ses mensurations incitent les auteurs à penser qu'il s'agit d'un Equus du Pléistocène moyen de moindre stature, «moins grand que certains chevaux rissiens et mindéliens " mais "sûrement plus élancé que l'Équidé de Lunel-Viel (interglaciaire Mindel / Riss)» (Guadelli et Prat 1995, p.95). Des caractères archaïques ont également été distingués. Le fait que cet Équidé possède un museau relativement court et une face haute est interprété comme une adaptation à un climat froid. «Le Cheval de Camp-de-Peyre (...) semble différer 
sensiblement de celui de Lunel-Viel, moins grand, aux formes lourdes, au museau modérément long et étroit qui, selon M.-F. Bonifay (1980), évoluait dans un milieu tempéré et humide » (Guadelli et Prat 1995 p.97).

De nombreux auteurs, comme Cl. Guérin (1996), M.-F. Bonifay (1980), V. Eisenmann et al. (1985) et J.-L. Guadelli et F. Prat (1995), font d'Equus mosbachensis une espèce à part entière qui comprendrait de nombreuses sous-espèces comme par exemple Equus mosbachensis campdepeyri, Equus mosbachensis palustris et Equus mosbachensis tautavelensis (cf. supra). Il nous paraît fortement probable qu'au sein de la lignée mosbachensis, qui a duré près de $500 \mathrm{ka}$, des sous-espèces soient apparues dans différentes régions. En effet, les Chevaux de la Caune de l'Arago, de Lunel-Viel et de Camp-de-Peyre possèdent des caractères morphologiques et biométriques relativement proches tout en ayant des particularités. C'est pourquoi nous pensons que ces Chevaux appartiennent à une même lignée : la lignée mosbachensis.

7 Lors de notre étude paléontologique, nous avons comparé les restes fauniques $d u$ Cheval de La Micoque à différents individus appartenant à l'espèce Equus mosbachensis mais également à d'autres chevaux plus récents appartenant à l'espèce caballus sensu stricto comme :

Equus caballus piveteaui David et Prat, 1962 : Equidé décrit à partir de fossiles mis au jour à l'Abri Suard en Charente. Il possède des caractères nettement caballins (David et Prat 1962) en ayant, cependant, gardé quelques caractères archaïques sur certaines mâchelières (Prat 1968). Cet Équidé, plus petit qu'Equus mosbachensis, présenterait des affinités avec ce dernier et serait d'une taille supérieure à celle d'Equus caballus germanicus (David et Prat 1962). Equus caballus piveteaui serait un descendant des grands chevaux apparentés à Equus mosbachensis tout en possédant des caractères qui lui sont spécifiques (David et Prat 1962 ; Prat 1968). Les gisements d'où sont issus les ossements attribués à cet Équidé ont été datés du «Riss III (Bordes et Prat 1965). Ce Cheval a aussi été reconnu à Combe-Grenal et Creyssac (Dordogne) ainsi qu'à l'Abri Suard (Charente) (Guadelli 1987).

9 Equus caballus germanicus Nehring, 1884: sur le site de Remagen en Allemagne, A. Nehring découvre en 1884, dans des niveaux datant de la dernière glaciation, des restes de chevaux qu'il attribue à une nouvelle sous-espèce : Equus caballus germanicus (in Prat 1968). Sa stature, ses pattes massives et ses larges sabots indiquent qu'il habitait dans « la bande préglaciaire froide et humide de l'Europe occidentale (Gromova 1949a, p. 126). Il possède en effet des os des extrémités d'une grande robustesse et $A$. Nehring (in Gromova 1949a) évalue sa hauteur au garrot à environ 1,55 m. Les restes de cet Équidé sont souvent associés à des faunes plus ou moins froides. Ce Cheval avait une aire de répartition qui s'étendait sur une grande partie de l'Europe occidentale. Dans le SudOuest de la France, nous pouvons citer de nombreux gisements dont La Chaise et La Quina en Charente, Combe-Grenal et le Pech-de-l'Azé en Dordogne et Pair-non-Pair en Gironde (Guadelli 1987; Prat 1968). Il est probablement présent dans le gisement d'Atapuerca (Espagne), où des restes de chevaux ont été attribués à Equus caballus $c f$. germanicus (Carbonell Roura et al. 1999). En France, Equus caballus germanicus est présent "durant une bonne partie du Paléolithique moyen et le début du Paléolithique supérieur» (Prat 1968 p.406). Il a été reconnu "dans les stations moustériennes du Würm II » (Prat op. cit. p.407) ainsi qu'au « début du Würm III » à La Chaise et à La Ferrassie. Son extension chronologique est sans doute comprise entre le stade isotopique $5 \mathrm{~d}$ et le stade isotopique 3 . 
10 Equus caballus gallicus Prat, 1968 : Un siècle après leur découverte, F. Prat étudie les restes d'un Équidé trouvés à Solutré (Saône-et-Loire) par A. Arcelin dès 1866 (Arcelin 1890). Il définit une nouvelle forme d'Équidé : Equus caballus gallicus (Prat 1968). D’une taille inférieure à celle d'Equus caballus germanicus (il ne mesurerait au garrot en moyenne que 1,35 à $1,38 \mathrm{~m}$ ), ce Cheval possédait une morphologie différente avec des caractères caballins mieux affirmés sur sa denture. Déjà en 1949, V. Gromova décrit le Cheval de Solutré comme un petit Équidé dont les dimensions des os sont proches de celles des Chevaux sauvages : Tarpan et Cheval de Przewalski. Cet Équidé apparaîtrait dès la fin de l'interstade Würm ancien / Würm récent, vers 35000 ans BP et perdurerait au moins jusqu'à 23 ou 24000 ans BP en Saône-et-Loire (Guadelli 1989). Equus caballus gallicus serait, d'après F. Prat (1968) et J.-L. Guadelli (1987), un descendant direct de Equus caballus germanicus. V. Eisenmann (1982) n'approuve pas totalement cette proposition et en 1991, elle rejette cette hypothèse et attribue tous les restes concernés à Equus caballus germanicus (Eisenmann 1991 p.752).

11 Equus caballus arcelini Guadelli, 1986: F. Prat (1968) pensait qu'un seul Cheval était présent à Solutré (Saône-et-Loire), mais dans les niveaux magdaléniens datés de 12500 ans BP, J.-L. Guadelli (1989) décrit un petit équidé possédant des jugales à très grand protocône. Cette forme est présente dans d'autres gisements du Würm supérieur : Le Queroy (Charente), Chasse-sur-Rhône (Isère) (Eisenmann 1991; Guadelli 1987 ; Guérin 1996). Ce Cheval possède des proportions à peu près identiques à celles d'Equus caballus gallicus, seule sa taille est plus faible (1,38 m pour arcelini et $1,41 \mathrm{~m}$ pour gallicus) (Guadelli 1989). Cette forme descendrait probablement d'Equus caballus gallicus. Ainsi le remplacement d'Equus caballus germanicus par Equus caballus gallicus puis par Equus caballus arcelini s'accompagnerait d'un changement de taille. D'après V. Eisenmann (1991), ce changement pourrait être le résultat d'une migration ou d'une évolution locale. Pour N. Spassov et N. Iliev (1997), il pourrait s'agir de migrations de chevaux, au cours de la glaciation würmienne, qui se seraient produites de l'Est de l'Europe vers l'Ouest.

12 A la suite de ces données, la lignée caballine peut être schématisée comme il est présenté dans le tableau 1.

Le Cheval, animal très souvent rencontré dans les faunes préhistoriques, a fait l'objet de nombreuses études qui ont abouti à diverses propositions systématiques.

F. Prat (1968) a été le premier à proposer une classification des Caballins en se fondant sur les variations de caractères évolutifs. D'autres arbres phylétiques ont également été proposés (cf. in Langlois 2004) comme par exemple celui d'A. Azzaroli (1982) ou la classification typologique de V. Eisenmann (1991). Ces classifications paraissent trop floues ou compliquent encore plus la lignée des chevaux. En effet, pour certaines filiations de l'arbre phylétique d'A. Azzaroli (1982), il manque des données et V. Eisenmann (1991), dans le sien, regroupe des espèces n'ayant aucun lien phylétique entre elles.

L'arbre phylétique établi d'après les travaux de F. Prat en 1968 complété par des informations tirées de travaux ultérieurs (Prat 1969 ; Guadelli et Prat 1995) servira de base à nos réflexions (tabl. 1). 
Tableau 1 - Évolution de quelques Équidés européens depuis $700 \mathrm{ka}$.

Table 1 - Evolution of some European Equids since $700 \mathrm{ky}$.

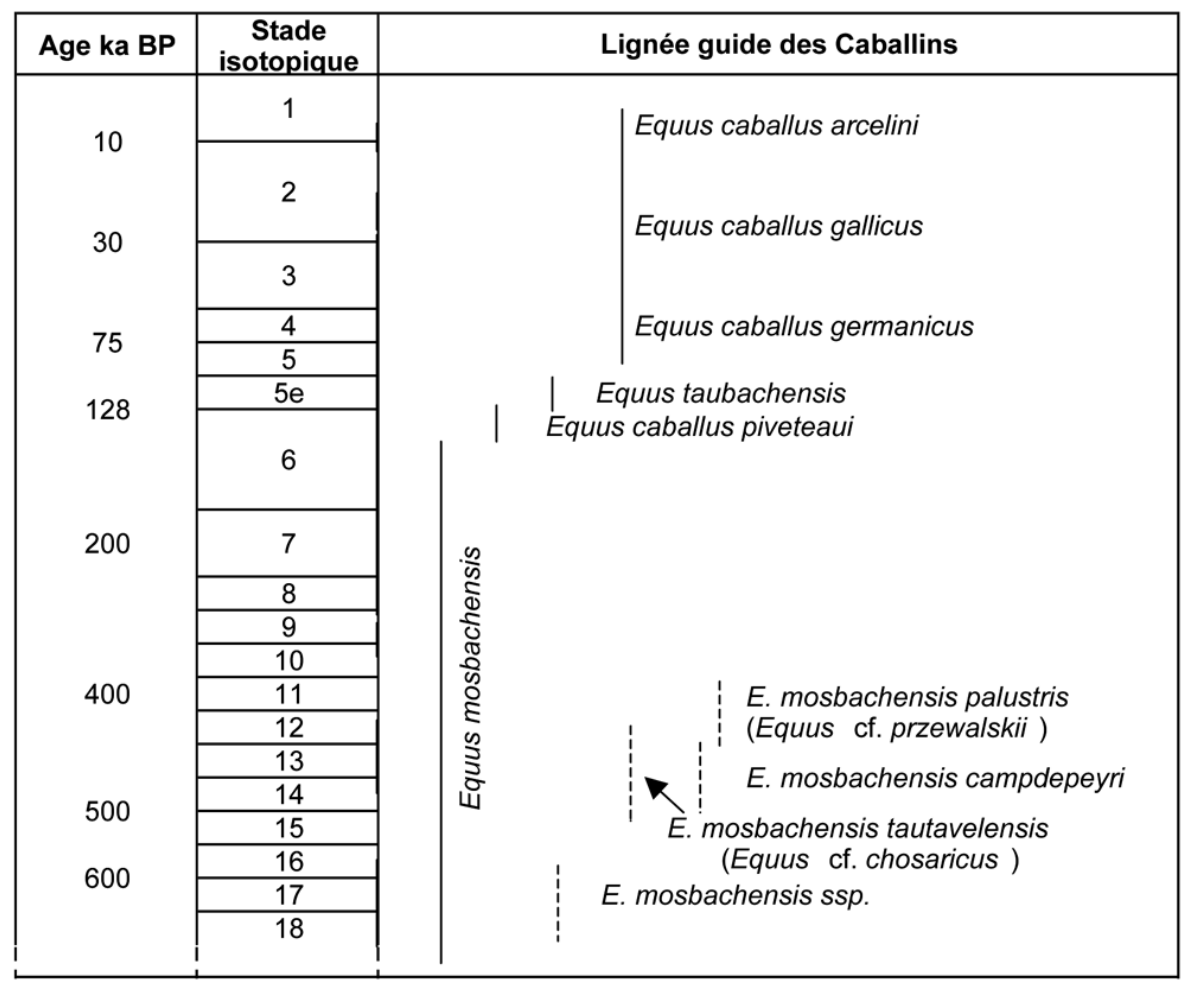

\section{2 - Présentation du gisement}

Le gisement de La Micoque (Les Eyzies-de-Tayac, Dordogne), fut découvert fortuitement il y a plus d'un siècle (fig.1). C'est en effet en 1895 que, lors d'un défrichement précédant la plantation d'une vigne, des ouvriers mirent au jour le site préhistorique. En 1896, G. Chauvet et R. Rivière entreprirent les premières fouilles (Chauvet et Rivière 1896, 1897). Au cours des années suivantes, de nombreux chercheurs se succédèrent, notamment D. Peyrony qui fouilla de 1929 à 1932 et identifia 15 couches et 6 niveaux archéologiques. Ce sont A. Debénath et J.-Ph. Rigaud, qui de 1983 à 1996, dirigèrent les dernières fouilles et exhumèrent le matériel sur lequel se fonde cet article (Rigaud 1984 ; Debénath et Rigaud 1986). 
Figure 1a - Localisation géographique du gisement de La Micoque (Dordogne). Figure 1a - Geographical localization of La Micoque (Dordogne).

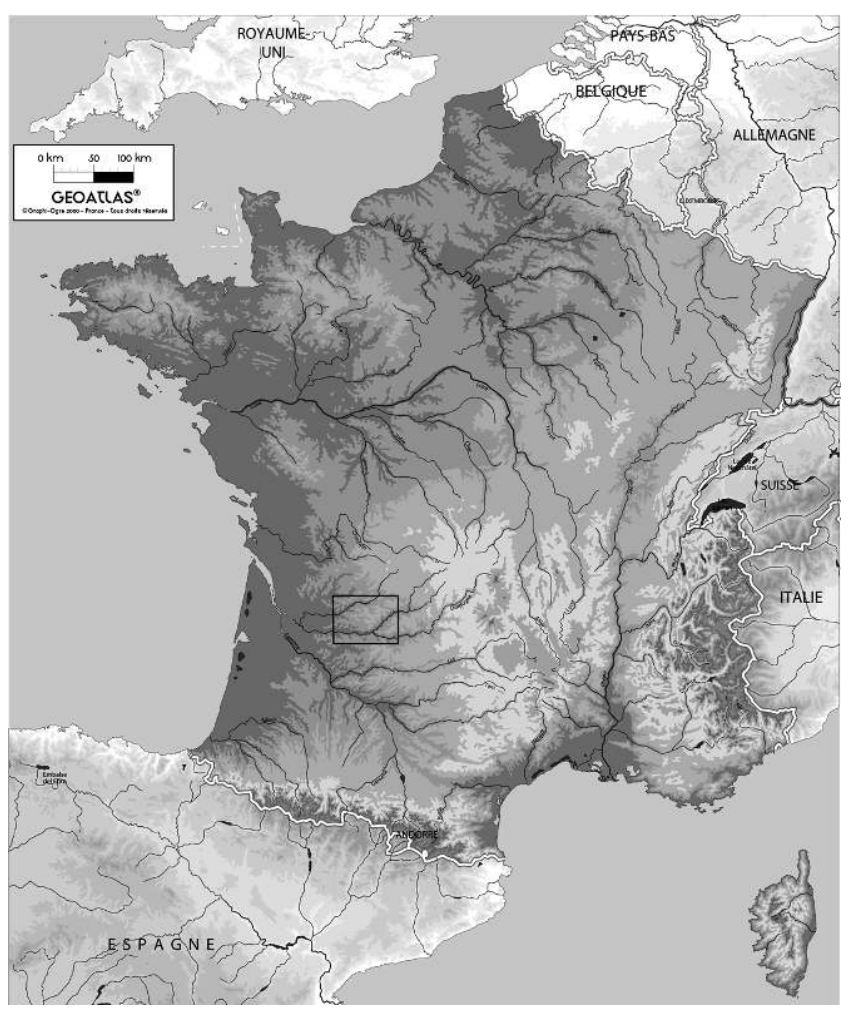

Figure $1 \mathrm{~b}$ - Localisation géographique du gisement de La Micoque (Dordogne). Figure $1 b$ - Geographical localization of La Micoque (Dordogne).

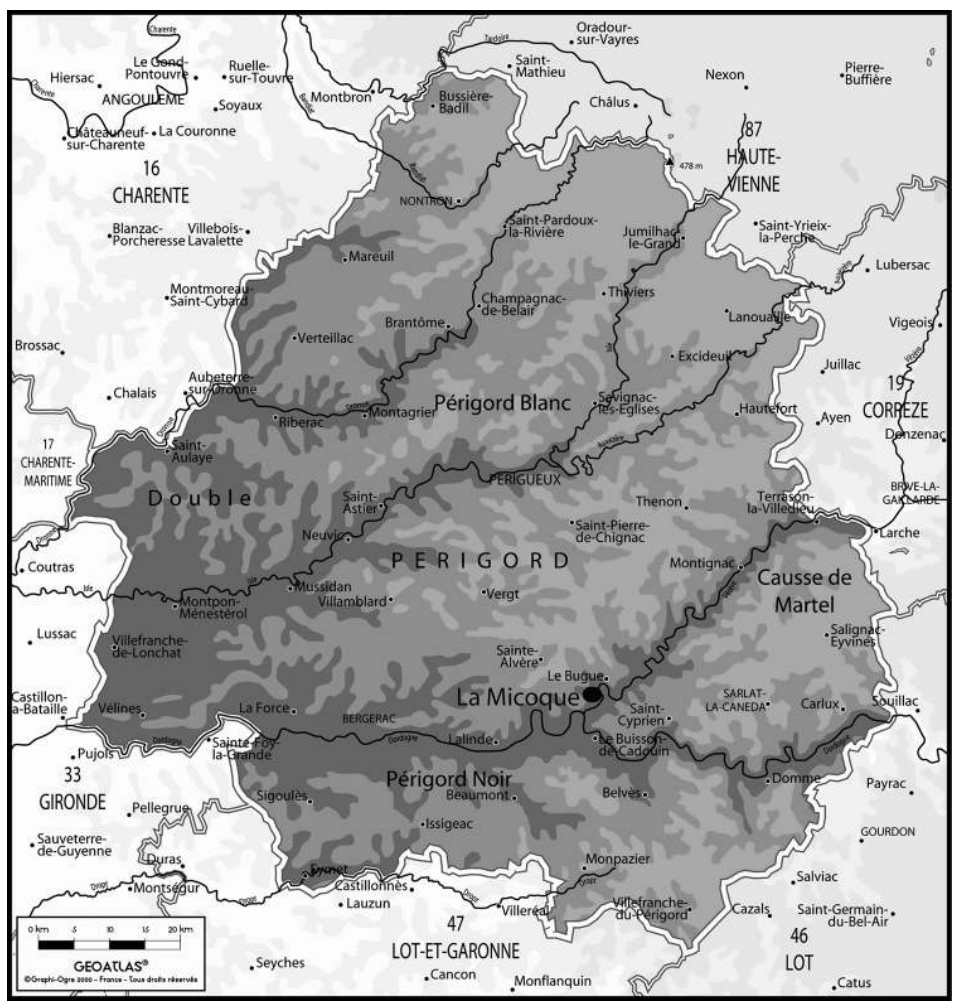



surtout en raison du développement de sa stratigraphie et de la richesse de plusieurs de ses niveaux en vestiges lithiques. Plusieurs strates livrèrent, en effet, des industries apparemment différentes de toutes celles reconnues jusqu'alors (niveaux archéologiques 2, 3, 4, 5, 5' et 6 des fouilles Peyrony correspondant respectivement aux couches $\mathrm{C}, \mathrm{E}, \mathrm{H}, \mathrm{J}$, L et N) (fig.2).

Figure 2 - Stratigraphie du gisement de La Micoque (in Laville et Rigaud 1976). Figure 2 - Stratigraphy of the site of La Micoque (in Laville and Rigaud 1976).

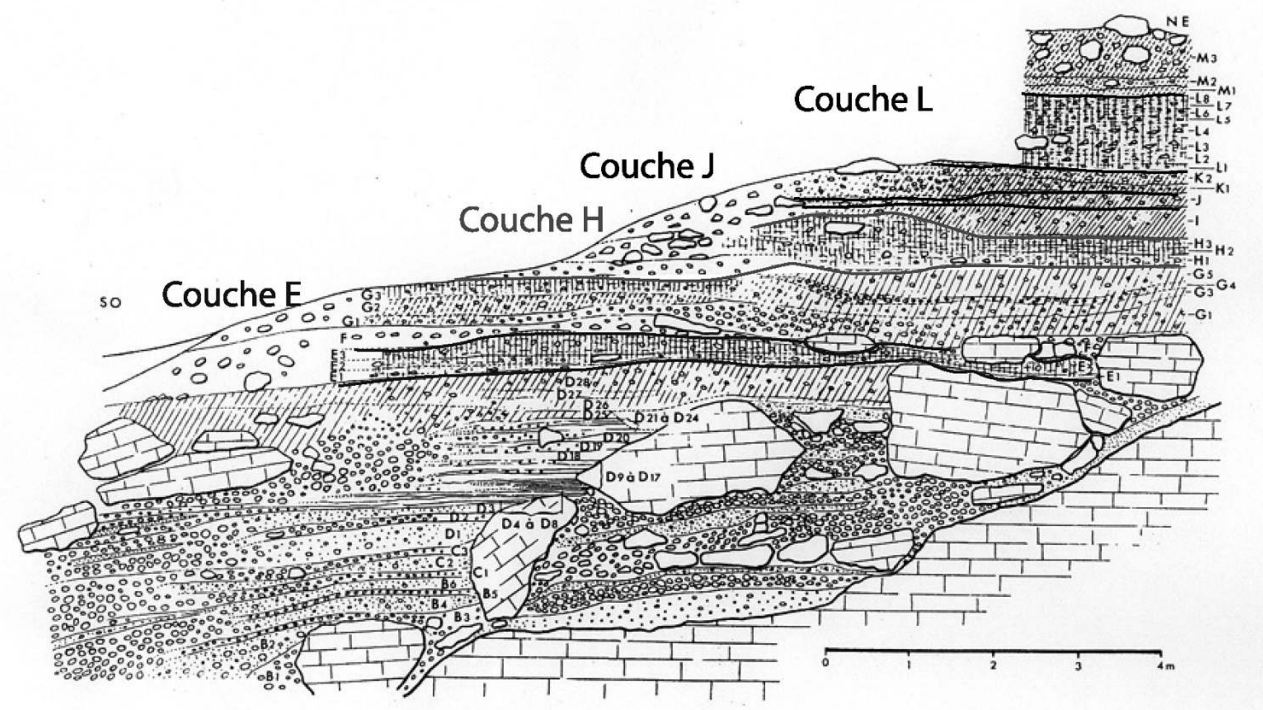

Les couches $E, H$, J et $L$ sont les couches fossilifères étudiées, la couche A n'est plus visible et la couche $\mathrm{N}$ a disparu.

Levels $E, H, J$ and $L$ are the fossiliferous levels studied, level $A$ is not apparent and level $N$ disappeared.

Dès 1916, le gisement de La Micoque devient site éponyme du « Micoquien » (Hauser 1916 in Leroi-Gourhan 1997). O. Hauser propose ce terme pour identifier au sein de la couche sommitale aujourd'hui disparue (niveau archéologique 6, couche $\mathrm{N}$ de la stratigraphie établie par D. Peyrony, 1938), une industrie composée de bifaces pointus et d'éclats préparés. Ce terme fut repris par H. Breuil en 1932.

deuxième industrie a été identifiée au sein des niveaux supérieurs par ce dernier auteur. Il s'agit des niveaux 4 et 5 qui correspondent aux couches $\mathrm{H}$ et $\mathrm{J}$ de la stratigraphie de H. Laville et J.-Ph. Rigaud (1976, fig.2). Cette industrie à débitage de type clactonien est associée à des racloirs et des denticulés "d'aspect très moustérien " à laquelle $\mathrm{H}$. Breuil attribua le terme de "Tayacien ». Des analyses récentes du matériel lithique ont montré que "les couches 3 et 4 de La Micoque peuvent être considérées comme moustériennes d'après leur outillage alors que la technologie du débitage et des bifaces les rapprocherait des industries de l'Acheuléen méridional » (Delpech et al. 1995, p.155). Les industries des couches 5, 5' et 6 ont des "caractères acheuléens plus marqués » (Delpech et al. 1995, p.156).

En ce qui concerne les datations radiométriques, les études de H. P. Schwarcz et R. Grün en 1988 et celles de C. Falguères et ses collaborateurs en 1997 ont montré que les couches fossilifères E à L2/3 ont un âge compris entre 300 et 350 ka (le niveau L2/3 est 
daté à $288 \pm 10$ ka par H. P. Schwarcz et R. Grün en 1988 et en 1997, C. Falguères et ses collaborateurs datent le même niveau à $284 \pm 20 \mathrm{ka}$ ), elles se seraient déposées lors d'une même période semi-aride froide, au cours du stade isotopique 10 (Texier et Bertran 1993).

Au cours des différentes campagnes de fouilles, l'originalité de ces techno-complexes a soulevé de nombreuses questions liées à la signification, en terme de culture, des techno-complexes eux-mêmes, à leur succession dans le temps et à leur répartition dans l'espace.

Avec le Pech-de-l'Azé II (Carsac, Dordogne), La Micoque constituait le deuxième gisement archéologique du Périgord comportant une longue séquence stratigraphique du Pléistocène moyen (Peyrony 1938). Bien que, lors des dernières décennies, soit venu s'ajouter un troisième site : la grotte XV de la falaise du Conte (Dordogne), dite grotte Vaufrey (Fouilles J.-Ph. Rigaud 1988), les sites du Pléistocène moyen restent peu nombreux par rapport à ceux du Pléistocène supérieur.

20 Enfin, le gisement de La Micoque est original car son spectre faunique (tabl. 2-annexe) est dominé par un taxon : le Cheval (plus de $88 \%$ du matériel déterminé), alors que les gisements voisins dont nous venons de parler (Vaufrey, Pech-de-l'Azé II) livrent une faune beaucoup plus variée (tabl. 3 et 4-annexe).

21 Les niveaux archéologiques sont regroupés d'un point de vue litho-stratigraphique dans les ensembles F4 (couches D, F, G, I à K) et DP3 (couches E, H et L) (fig.2 et 3). L'ensemble F4 d'origine fluviatile, s'oppose à DP3 dont les sédiments proviennent essentiellement du versant (Texier et Bertran 1993).

Figure 3 - Litho-stratigraphie du site de La Micoque (d'après Texier et Bertran 1993). Figure 3 - Litho-stratigraphy of La Micoque (after Texier \& Bertran 1993).

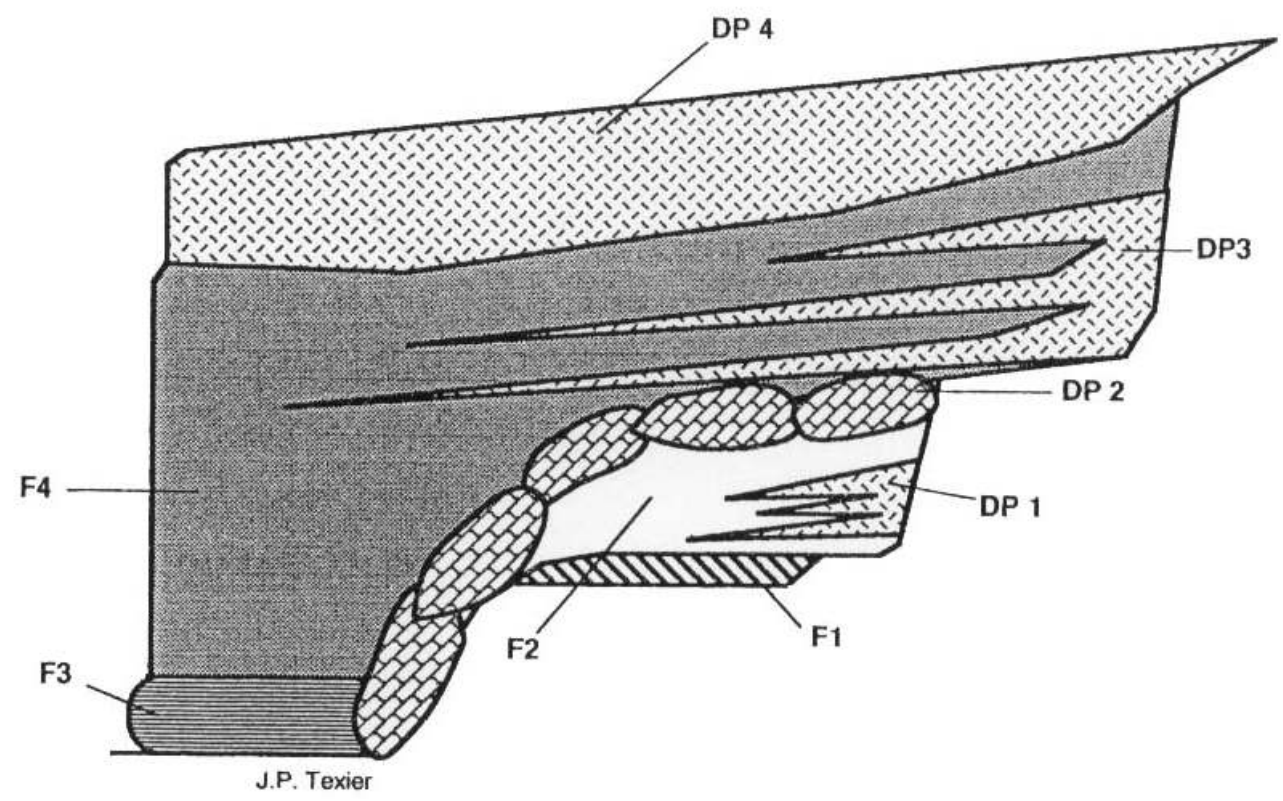

Ensemble inférieur (F1, F2, DP1) stérile ; Ensemble moyen (F3, F4, DP3) regroupe les couche A à N de D. Peyrony ; Ensemble supérieur (DP4) a livré quelques silex à sa base, non décrit précédemment. Lower unit (F1, F2, DP1) sterile; middle unit (F3, F4, DP3) including the layers A to N of D. Peyrony; Upper unit (DP4) displaying in its lower part, some silex, not previously described. 


\section{3 - Étude paléontologique}

\section{1 - Présentation du matériel} supérieures, on note : des faces vestibulaires du paracône et du métacône très concaves, un protocône bilobé possédant un sillon médial plus ou moins marqué, un mésostyle dédoublé et assez large sur les prémolaires et molaires, un parastyle souvent dédoublé sur les prémolaires. Sur les jugales inférieures : une double boucle dissymétrique, un métastylide triangulaire et un métaconide pédonculé et globuleux, un sillon lingual profond et en forme de $\mathrm{U}$, un sillon vestibulaire peu profond.

A partir de l'étude morphologique des dents, nous pouvons dire que le Cheval présent à La Micoque appartient au groupe des « vrais » chevaux : les Caballins.

\section{P2 supérieures}

Les deuxièmes prémolaires supérieures (P2/) du Cheval de La Micoque ne s'individualisent pas de celles des autres Chevaux (fig.4) (Langlois 2004, tabl. 22). 
Figure 4 - P2 supérieures de différents gisements.

Figure 4 - Upper $P 2$ from various sites.

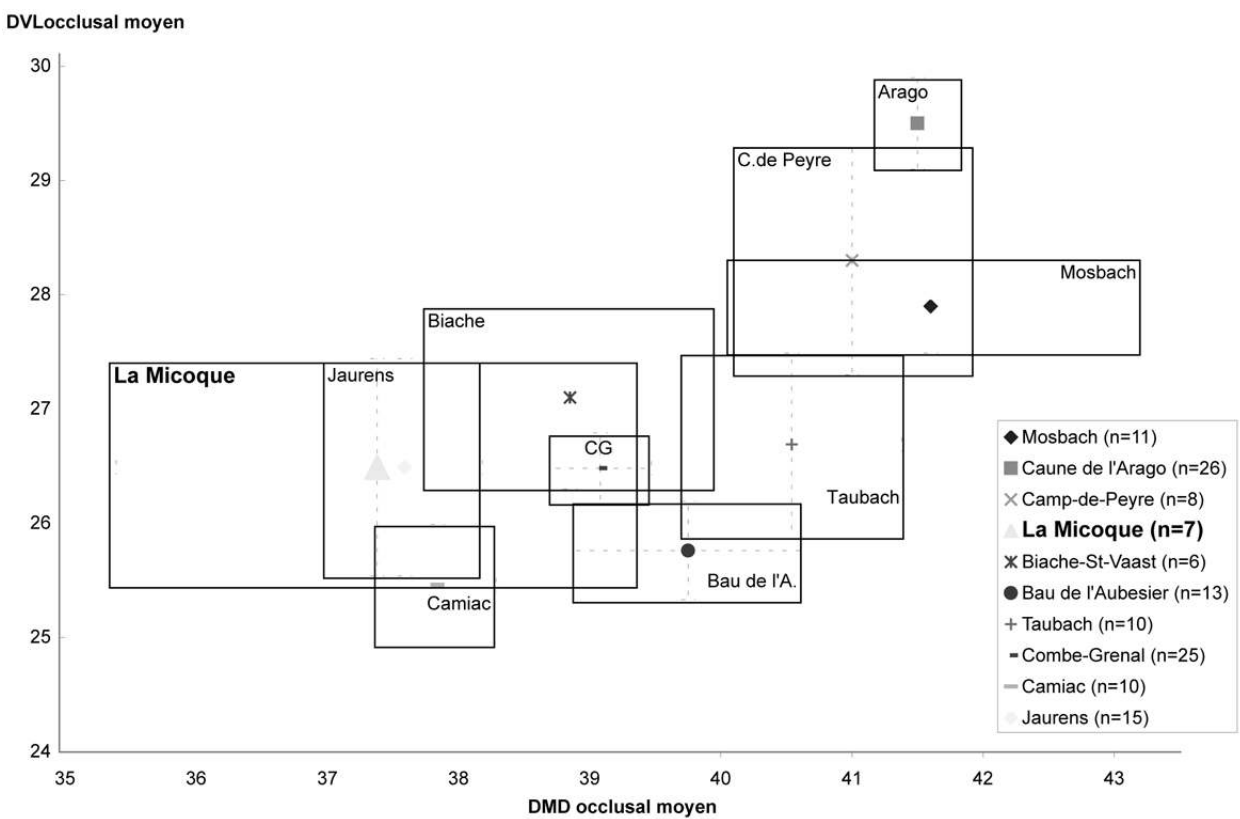

Diamètres mésio-distal et vestibulo-lingual moyens occlusaux et leurs intervalles de confiance à 95\%. Average mesio-distal and vestibulo-lingual occlusal diameters and their reliable intervals up to $95 \%$.

Le diamètre mésio-distal (DMD) ${ }^{1}$ du protocône de ces P2/ (tabl. 6-annexe) est peu différent de celui des chevaux de Biache-Saint-Vaast (Pas-de-Calais), du Bau de l'Aubesier (Vaucluse) et même de Combe-Grenal et de Camiac. Toutefois, relativement au diamètre mésio-distal occlusal de la dent, il est beaucoup plus court que chez ces derniers (fig.5). 
Figure 5 - P2 supérieures. Diamètre mésio-distal moyen occlusal du protocône en fonction du diamètre mésio-distal moyen occlusal de la dent.

Figure 5 - Upper P2. Average mesio-distal occlusal diameter of protocone in terms of average mesiodistal diameter of teeth.

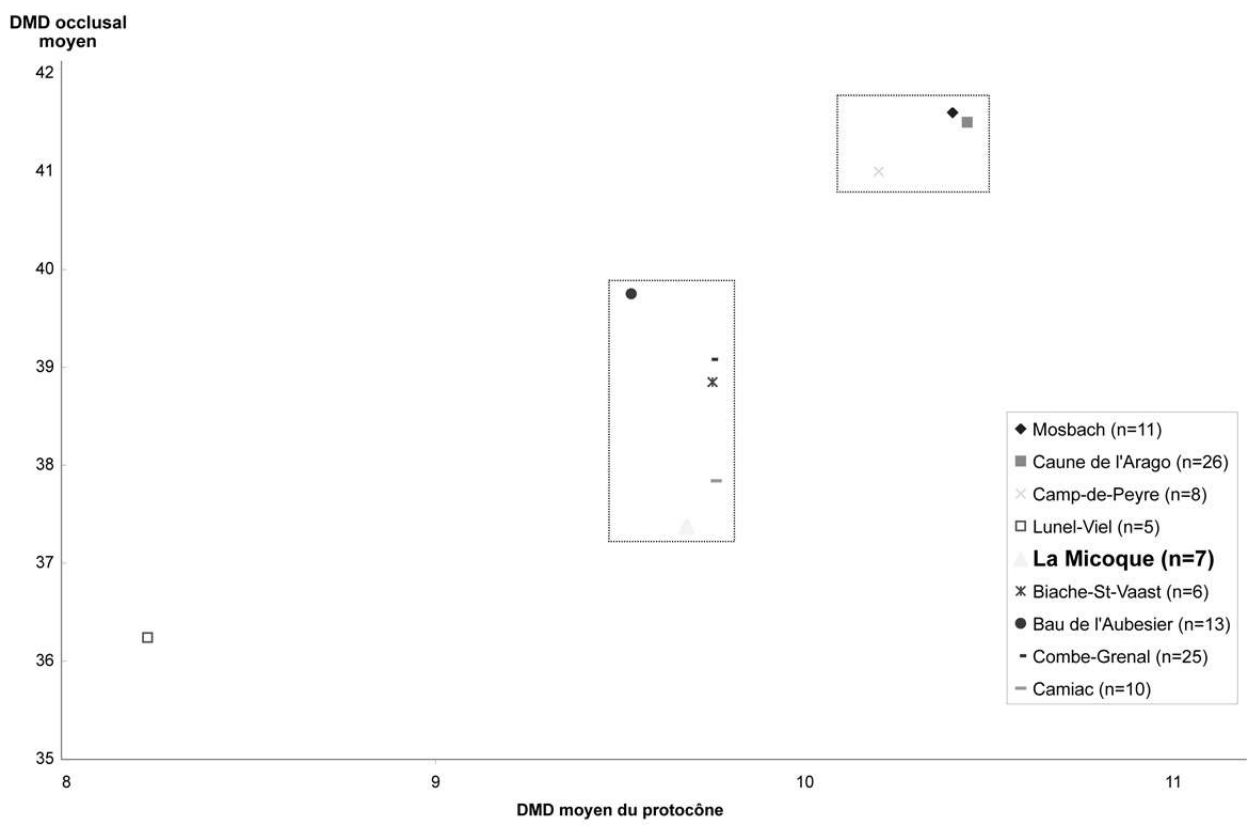

Les deuxièmes prémolaires supérieures du Cheval de La Micoque sont donc parmi les plus petites mesurées. En cela, elles sont proches de celles d'Equus mosbachensis de Biache-Saint-Vaast, Bau de l'Aubesier mais également proche de celles d'Equus caballus germanicus de Combe-Grenal et d'Equus caballus gallicus de Camiac.

\section{P3-4 supérieures}

Les Chevaux des sites de Mosbach, de la Caune de l'Arago et de Camp-de-Peyre possèdent des troisièmes et quatrièmes prémolaires supérieures dont la surface occlusale est très vaste (Langlois 2004, tabl. 23). En revanche, les P3-4 des Chevaux de Biache-Saint-Vaast, du Bau de l'Aubesier et de Taubach (Allemagne) ainsi qu'à CombeGrenal et Camiac présentent des diamètres mésio-distal et vestibulo-lingual courts. Ces dimensions sont comparables à celles des prémolaires 3 et 4 du Cheval de La Micoque (tabl. 7- annexe, fig.6). 
Figure 6 - P3-4 supérieures de différents gisements. Diamètres mésio-distal et vestibulo-lingual moyens occlusaux et leurs intervalles de confiance à $95 \%$.

Figure 6 - Upper P3-4 from various sites. Average mesio-distal and vestibulo-lingual occlusal diameters and their reliable intervals up to $95 \%$.

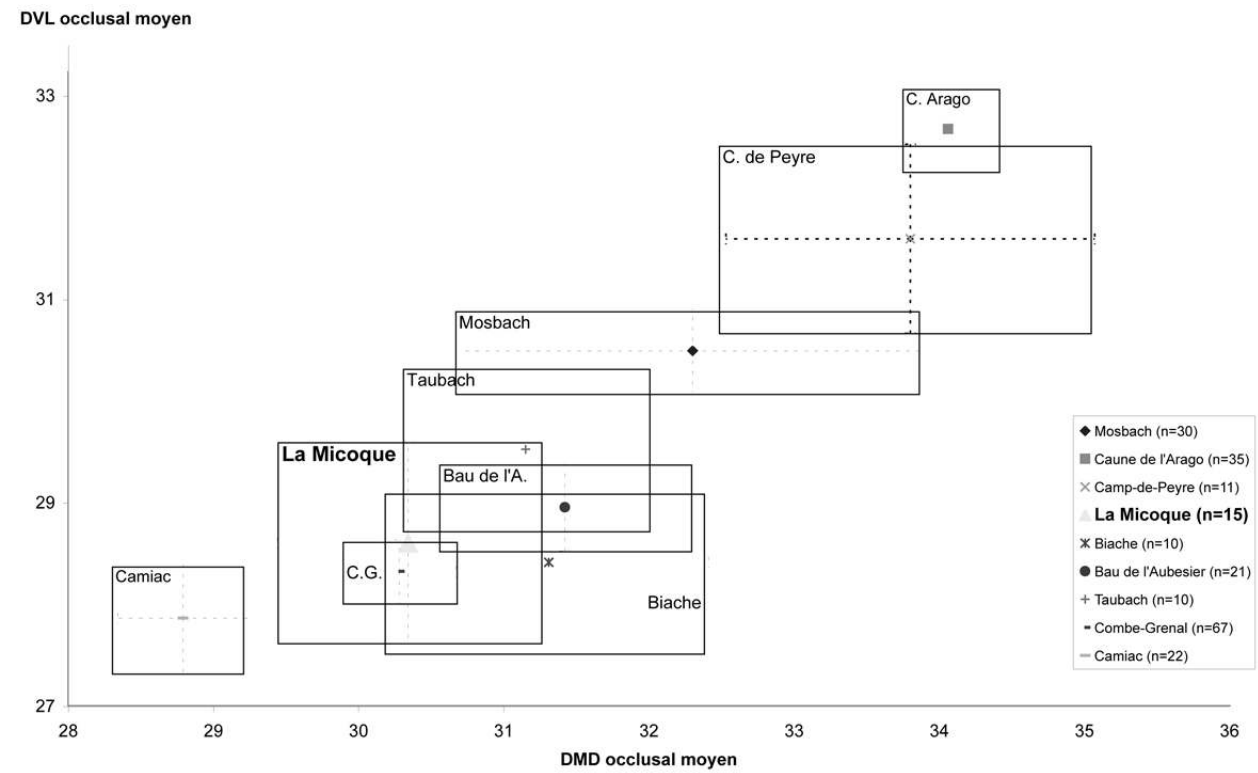

31 Mis à part le Cheval de Lunel-Viel qui a un protocône très court, le Cheval de La Micoque, comme tous les autres chevaux auxquels on le compare, présente des prémolaires avec un diamètre mésio-distal (DMD) du protocône relativement important par rapport au diamètre mésio-distal de la dent (fig.7).

Figure 7 - P3-4 supérieures. Diamètre mésio-distal moyen occlusal du protocône en fonction du diamètre mésio-distal moyen occlusal de la dent.

Figure 7 - Upper P3-4. Average mesio-distal occlusal diameter of protrocone in terms of average mesiodistal occlusal diameter of teeth.

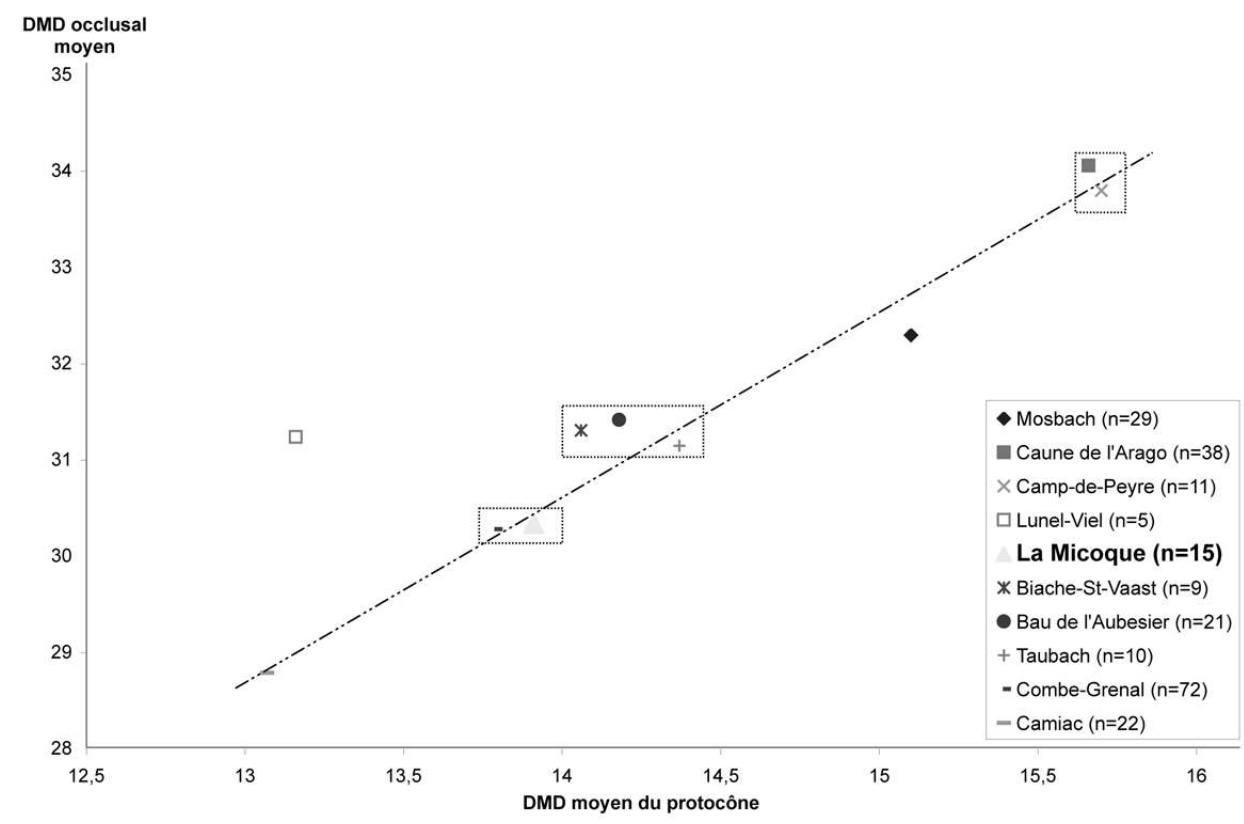


L'étude biométrique des restes dentaires permet de montrer que les prémolaires supérieures des Chevaux de La Micoque ont des dimensions relativement petites (tabl. 7-annexe) comme chez les chevaux de Biache-Saint-Vaast et du Bau de l'Aubesier.

\section{M1-2 supérieures}

Les M1-2 supérieures du Cheval de La Micoque présentent également des particularités d'un point de vue dimensionnel: leur diamètre vestibulo-lingual (DVL) est faible relativement à leur diamètre mésio-distal (tabl. 8-annexe) (Langlois 2004, tabl. 24). Ainsi, si du point de vue de leur longueur elles se rapprochent des grands chevaux de Camp-de-Peyre et de la Caune de l'Arago, elles sont cependant beaucoup plus étroites. De part ces caractères, les M1-2 supérieures du Cheval de La Micoque se rapprochent beaucoup de celles du Cheval de Taubach (fig.8).

Figure 8 - M1-2 supérieures de différents gisements.

Figure 8 - Upper M1-2 from various sites.

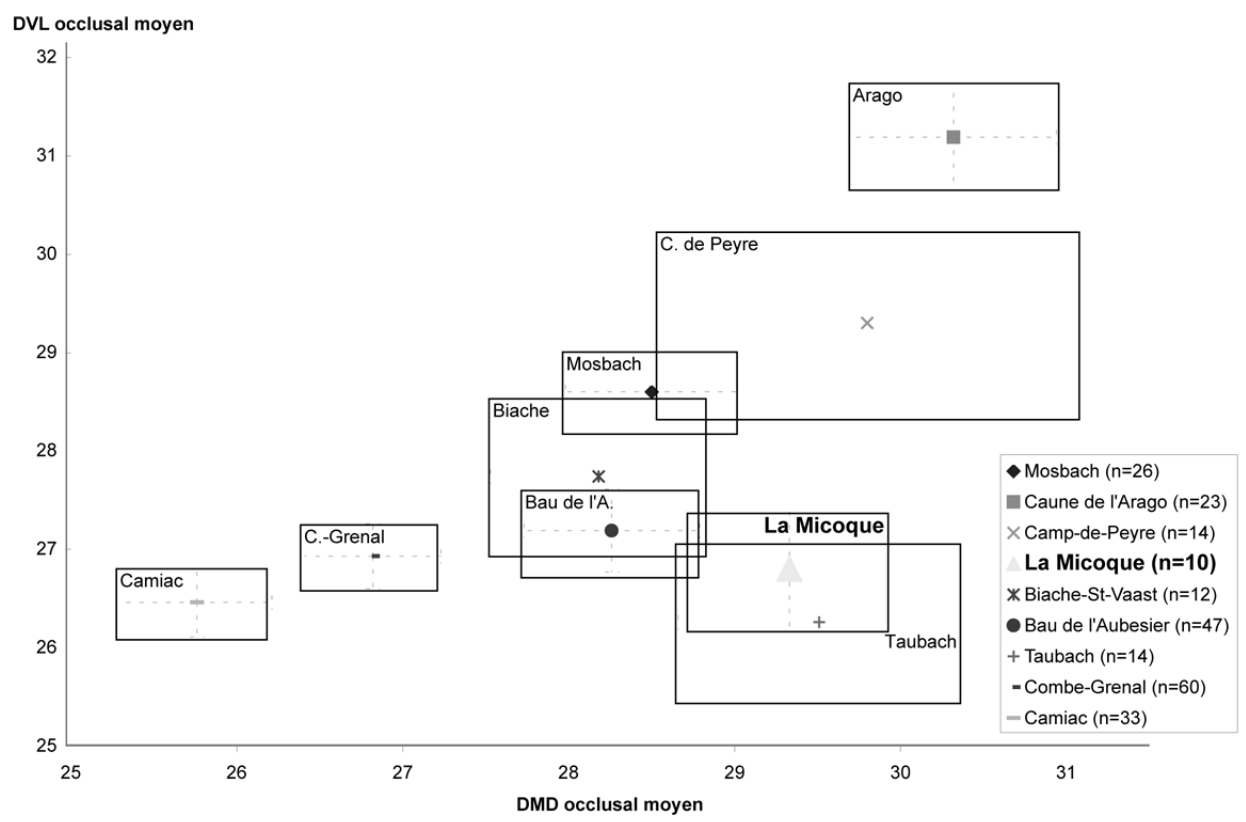

Diamètres mésio-distal et vestibulo-lingual moyens occlusaux et leurs intervalles de confiance à $95 \%$.Average mesio-distal and vestibulo-lingual occlusal diameter and their reliable intervals up to $95 \%$.

Le diamètre mésio-distal (DMD) du protocône de ces molaires est relativement court par rapport à ce qu'on peut voir chez les " grands chevaux ", en cela le Cheval de La Micoque est proche du Cheval de Lunel-Viel. On remarque que le protocône de ses M1-2 supérieures n'est pas plus long que celui des M1-2 supérieures du Cheval de Camiac dont la longueur des molaires (DMD) est pourtant beaucoup plus faible (fig.9). 
Figure 9 - M1-2 supérieures de différents gisements. Figure 9 - Upper M1-2 from various sites.

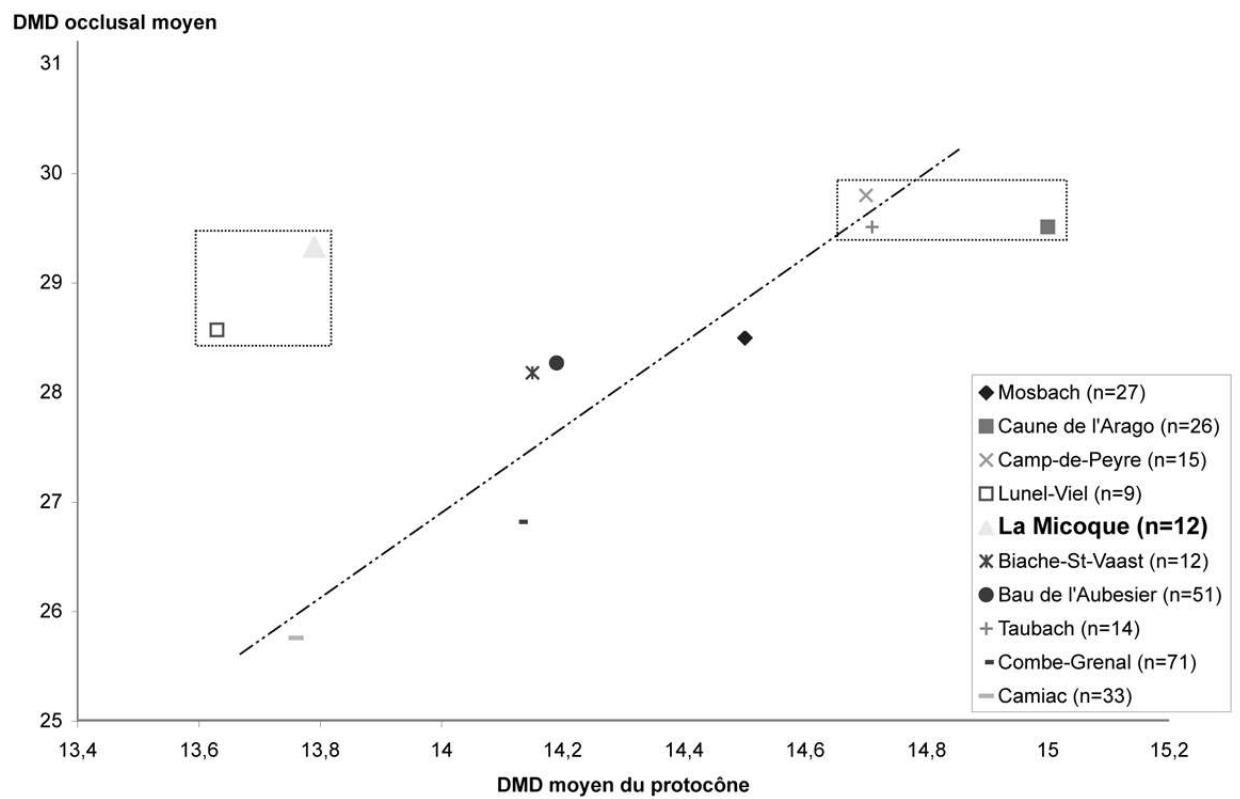

Diamètre mésio-distal moyen occlusal du protocône en fonction du diamètre mésio-distal moyen occlusal de la dent.

Average mesio-distal occlusal diameter of protocone in terms of average mesio-distal occlusal diameter of teeth.

\section{M3 supérieures}

Les troisièmes molaires supérieures des chevaux de La Micoque possèdent des dimensions comparables à celles des Equus mosbachensis (fig.10) (Langlois 2004, tabl. 25). Elles sont massives avec un protocône très court (tabl. 9-annexe). C'est également le cas chez l'Equus caballus gallicus de Camiac (fig.11), de même que sur les M3 supérieures du Cheval de Biache-Saint-Vaast. 
Figure $10-M 3$ supérieures de différents gisements. Figure 10 - Upper M3 from various sites.

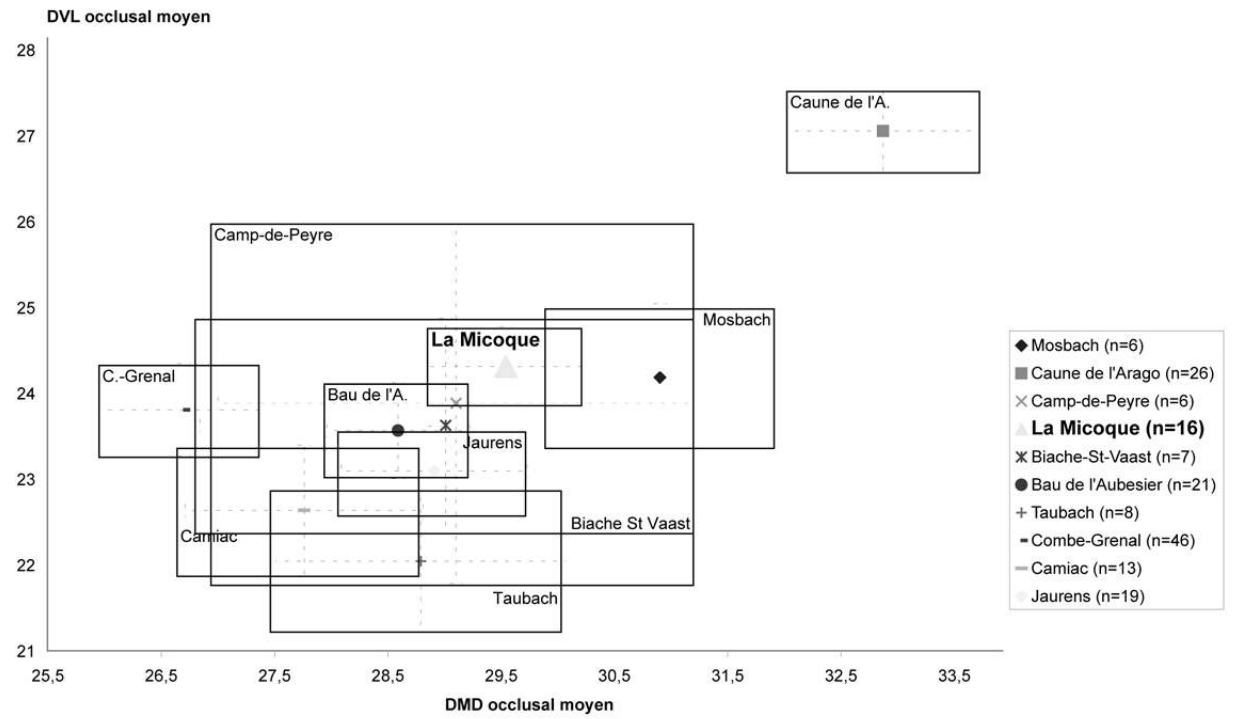

Diamètres mésio-distal et vestibulo-lingual moyens occlusaux et leurs intervalles de confiance à $95 \%$. Average mesio-distal and vestibulo-lingual occlusal diameters and their reliable intervals up to $95 \%$.

Figure 11 - M3 supérieures de différents gisements.

Figure 11 - Upper M3 from various sites.

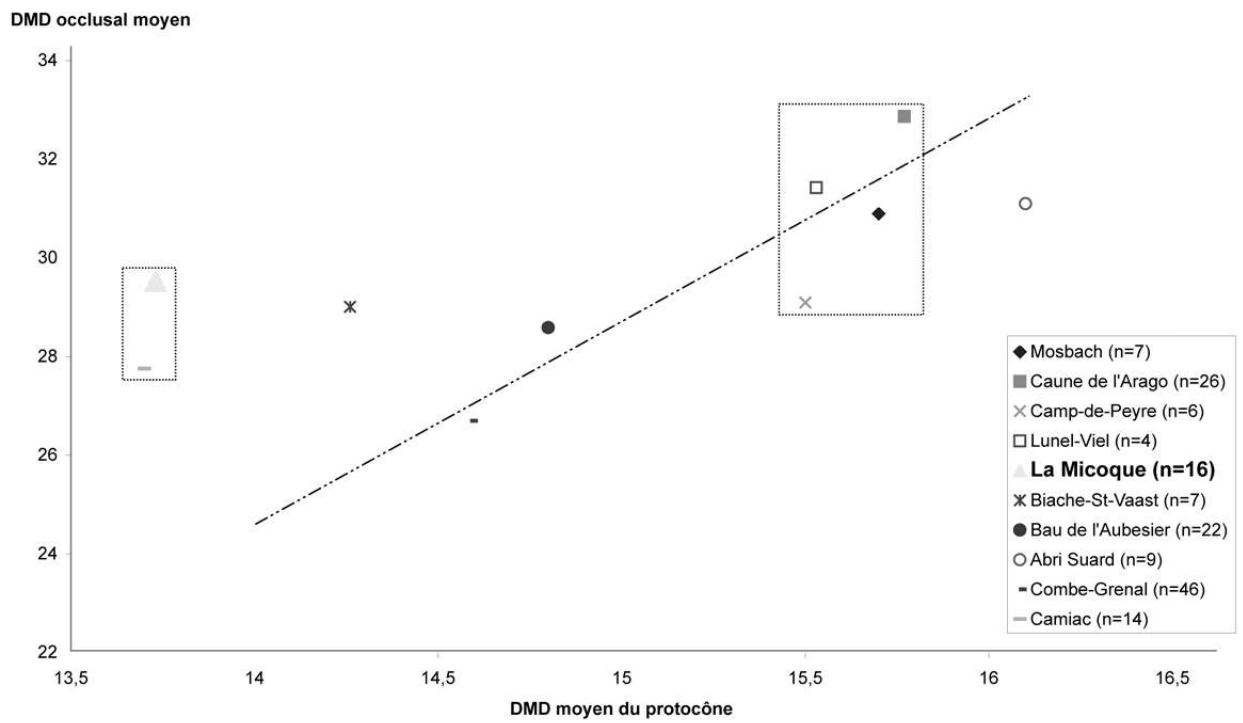

Diamètre mésio-distal moyen occlusal du protocône en fonction du diamètre mésio-distal moyen de la dent.

Average mesio-distal occlusal diameter of protocone in terms of average mesio-distal occlusal diameter of teeth.

En définitive, le Cheval de La Micoque possède des prémolaires de petite taille, des première et deuxième molaires aussi longues (diamètre mésio-distal) que celles des plus grands chevaux mais beaucoup plus étroites (diamètre vestibulo-lingual) alors que la troisième molaire ne diffère pas de celle des plus grands Equus mosbachensis. Quant au 
protocône, il ne présente pas de particularités sur les prémolaires mais il est très court sur les molaires (tabl. 10-annexe).

\section{Évolution de l'indice protoconique au sein de la série des dents jugales supérieures}

Pour rappel, l'indice protoconique représente, pour chaque dent, la longueur du diamètre mésio-distal du protocône relativement à celle du diamètre mésio-distal occlusal, le tout multiplié par 100. Cette évolution serait différente chez les chevaux de type mosbachensis et chez ceux du type caballus : l'indice augmenterait de la P2/ à la M3/ chez les premiers et chute au niveau de la M3/ chez les seconds. Cette particularité a été démontrée par V. Eisenmann en 1980 et nous la retrouvons pour les différents Équidés utilisés ici comme référents. Les figures 12 et 13 montrent clairement que :

1. pour Camp-de-Peyre et la Caune de l'Arago, l'indice protoconique présente une élévation régulière de $\mathrm{M} 1$ à $\mathrm{M} 3$;

2. pour Lunel-Viel et le Bau de l'Aubesier, l'élévation est forte au niveau des M1-2 puis plus faible pour M3 ;

3. pour La Micoque, l'augmentation est régulière mais faible ;

4. pour Biache-Saint-Vaast, Taubach et Combe-Grenal, l'indice protoconique diminue légèrement de $\mathrm{M} 1-2$ vers $\mathrm{M} 3$;

5. pour l'Abri Suard et Camiac, la baisse de l'indice protoconique au niveau des M3 est forte. Il existe une distinction nette entre le groupe des «grands » chevaux de type mosbachensis (auxquels on peut ajouter Equus taubachensis) et celui des chevaux plus récents: Equus caballus piveteaui, Equus caballus germanicus et Equus caballus gallicus. L'évolution de l'indice protoconique des dents jugales du Cheval de La Micoque rapproche celui-ci des formes « anciennes » du Pléistocène moyen.

Figure 12 - Indice protoconique moyen des jugales supérieures de Equus mosbachensis et de Equus taubachensis comparé à celui des jugales supérieures du Cheval de La Micoque.

Figure 12 - Average protoconic coefficient of Equus mosbachensisand Equus taubachensis upper cheek-teeth compared to the protoconic coefficient of the Horse of La Micoque upper cheek-teeth.

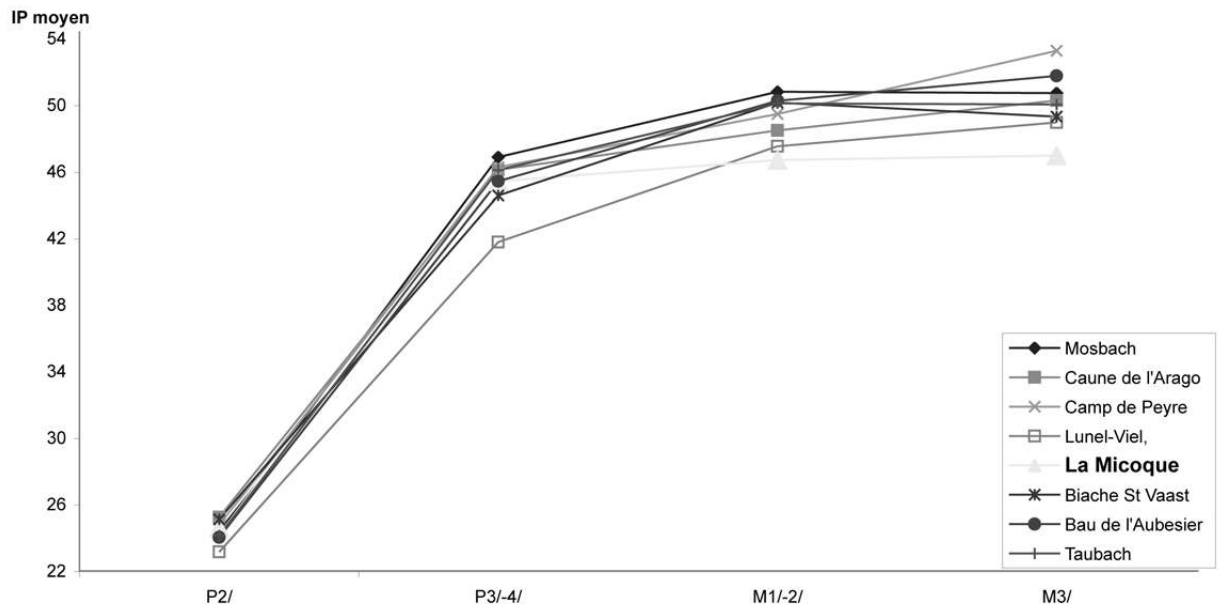


Figure 13 - Indice protoconique des jugales supérieures des Équidés de type piveteaui, germanicus et gallicus comparé à celui des jugales supérieures du Cheval de La Micoque.

Figure 13 - Protoconic coefficient of upper cheek-teeth of E. caballus piveteaui, E. caballus germanicus and $\mathrm{E}$. caballus gallicus compared to the protoconic coefficient of the Horse of La Micoque upper cheek-teeth.

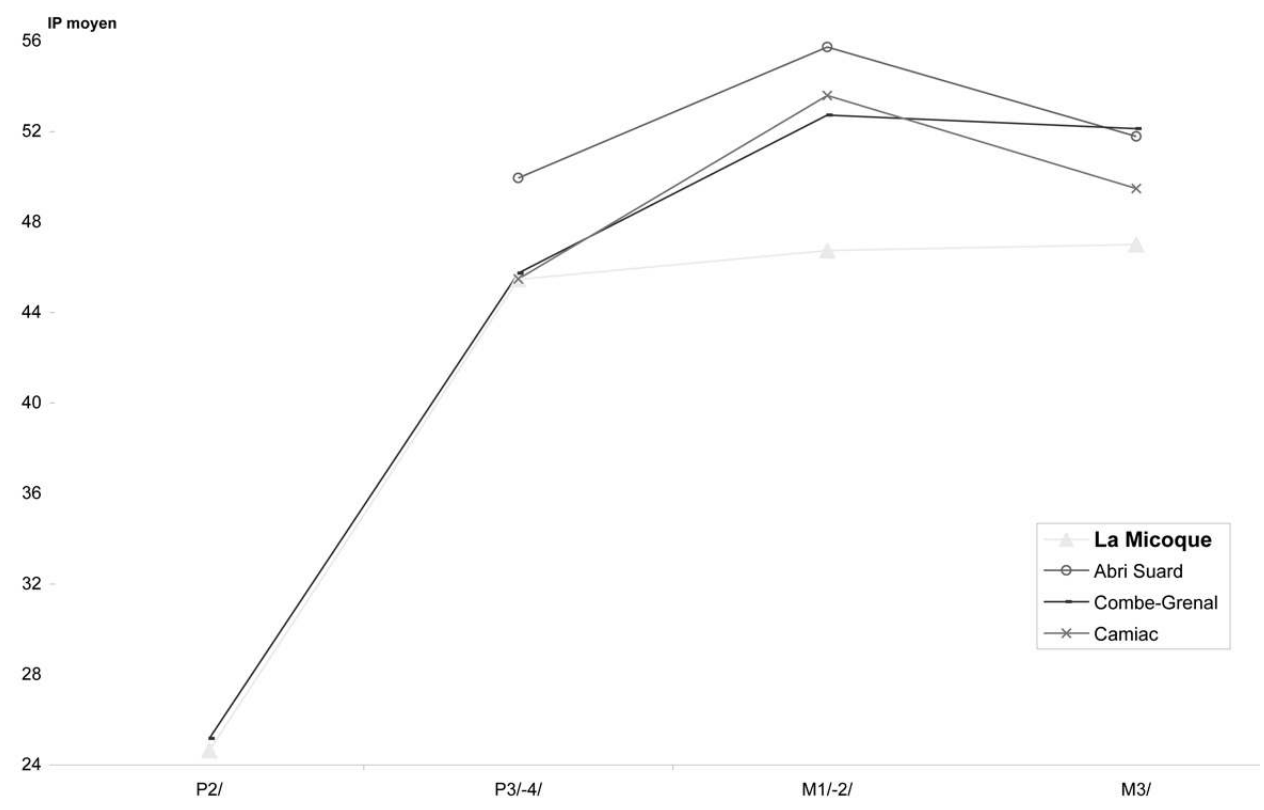

Le diamètre mésio-distal des dents serait lié au type de végétation dont les individus tirent leur nourriture. V. Gromova (1949a) et de nombreux autres auteurs (Guadelli et Prat 1995 ; Eisenmann 1982) s'accordent à dire que l'allongement du protocône (et l'accroissement de l'hypsodontie) des dents correspondraient à une végétation de type steppique. Le Cheval de La Micoque présente des jugales supérieures dont les diamètres mésio-distaux des protocônes sont relativement assez courts par rapport à ceux de la Caune de l'Arago, Camp-de-Peyre et Mosbach. Les chevaux de La Micoque, Lunel-Viel (fig.9) et Camiac (fig.11), devaient vivre dans un environnement moins steppique que les chevaux cités précédemment (Bonifay 1980 ; Guadelli 1987).

\section{P2 inférieures}

Les deuxièmes prémolaires inférieures présentent toutes un diamètre vestibulo-lingual (DVL) relativement faible (Langlois 2004, tabl. 27). Les dents du Cheval de La Micoque se distinguent par un diamètre mésio-distal relativement élevé (fig.14 - tabl. 11-annexe). Seuls, les Chevaux de la Caune de l'Arago possèdent des dents de très grandes dimensions. Le Cheval de La Micoque, par la taille de ces $\mathrm{P} / 2$, se situe entre ceux de Camp-de-Peyre et Mosbach d'une part et de ceux du Bau de l'Aubesier et de CombeGrenal d'autre part. 
Figure 14 - P2 inférieures de différents gisements. Figure 14 - Lower $P 2$ from various sites.

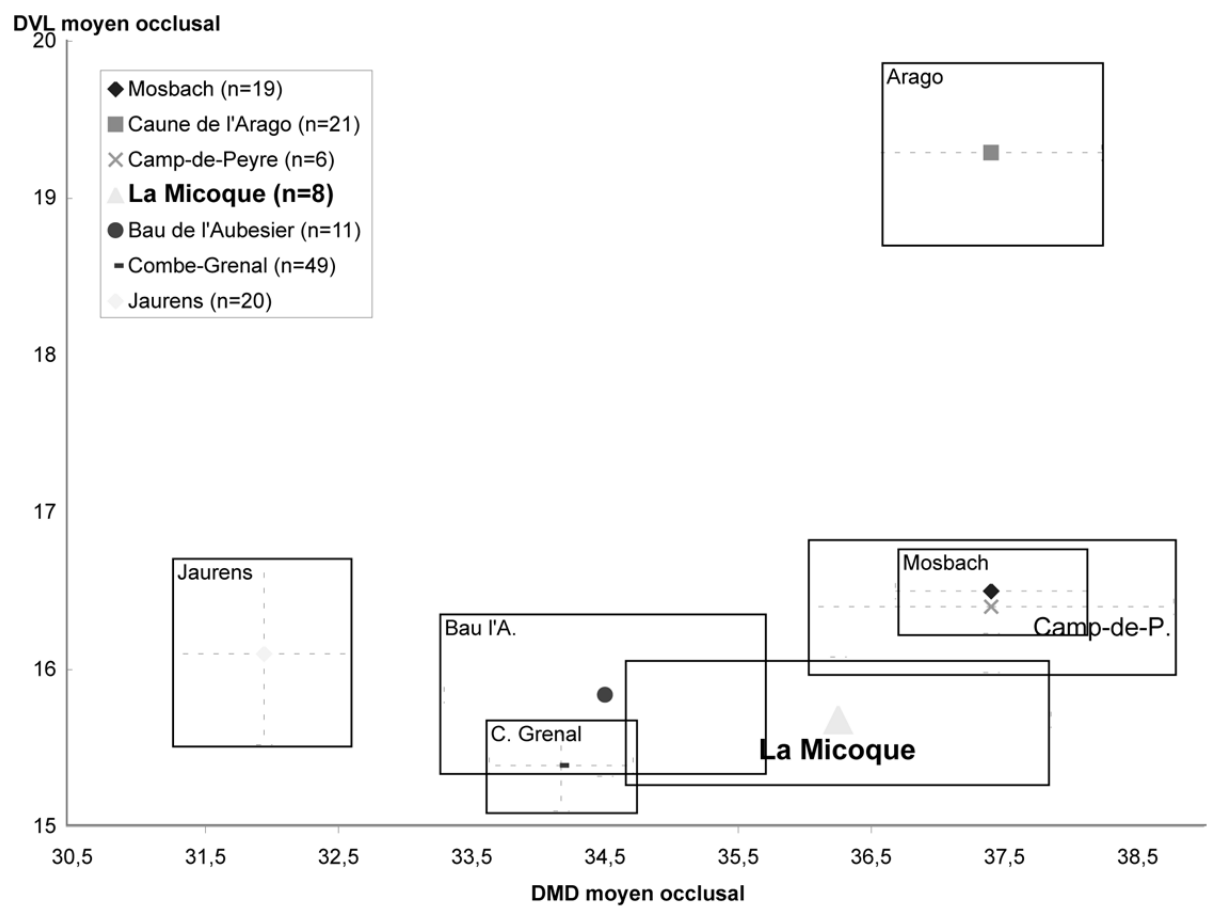

Diamètres mésio-distal et vestibulo-lingual moyens occlusaux et intervalles de confiance à $95 \%$. Average mesio-distal and vestibulo-lingual occlusal diameters and their reliable intervals up to $95 \%$.

\section{P3-4 inférieures}

Les P3-4 inférieures ont toutes un diamètre vestibulo-lingual relativement faible (tabl. 12 - annexe) (Langlois 2004, tabl. 28), seuls les Chevaux de Caune de l'Arago et Camp-de-Peyre présentent des prémolaires dont les diamètres vestibulo-lingual et mésio-distal sont élevés (fig.15). Le Cheval de La Micoque possède des prémolaires de longueur intermédiaire entre d'une part les chevaux de la Caune de l'Arago, Camp-dePeyre et Mosbach et d'autre part les chevaux du Bau de l'Aubesier et de Taubach. Toutefois, les variations de dimensions du diamètre vestibulo-lingual ne sont pas très importantes entre les Chevaux de Combe-Grenal et ceux de La Micoque. 
Figure 15 - P3-4 inférieures de différents gisements. Figure 15 - Lower P3-4 from various sites.

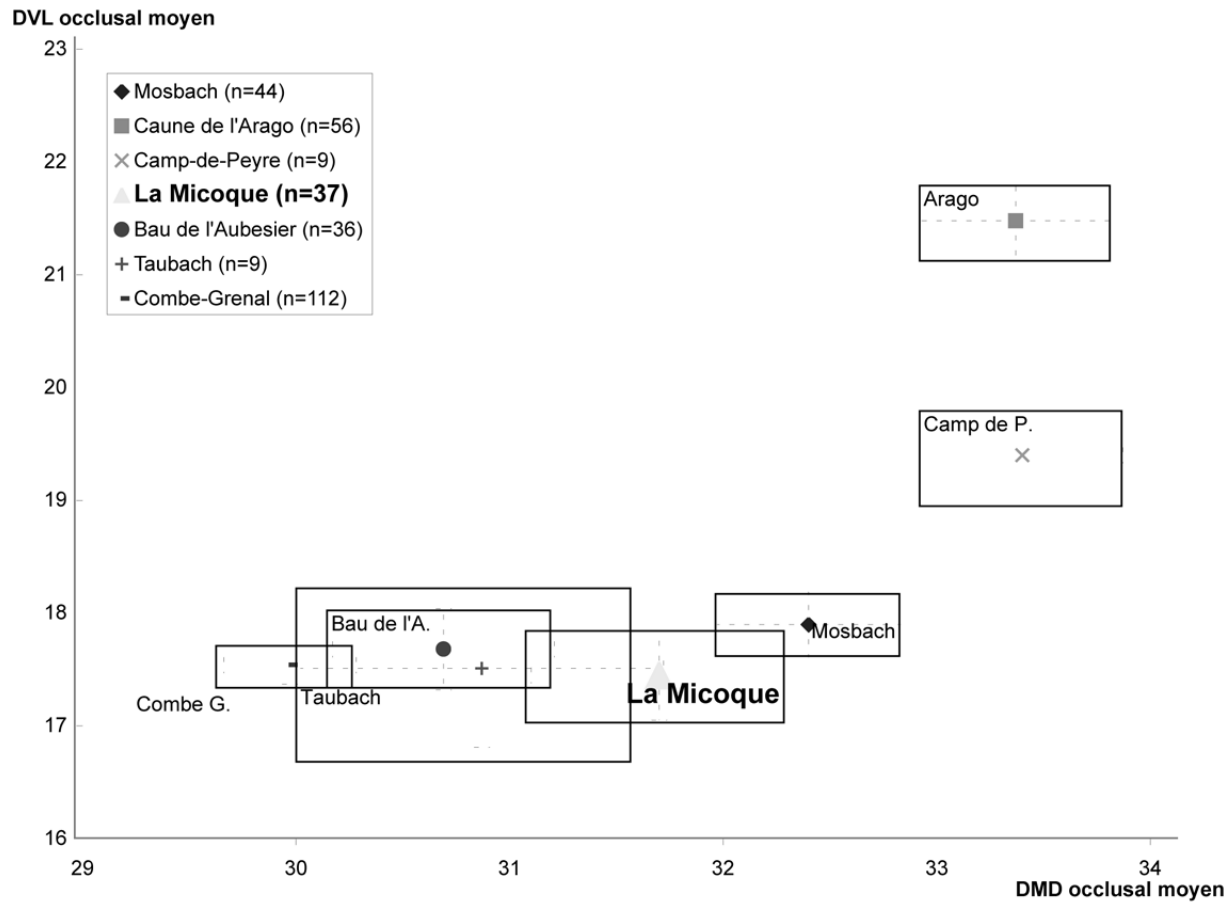

Diamètres mésio-distal et vestibulo-lingual moyens occlusaux et intervalles de confiance à $95 \%$. Average mesio-distal and vestibulo-lingual occlusal diameters and their reliable intervals up to $95 \%$.

\section{M1-2 inférieures}

41 Les premières et deuxièmes molaires inférieures du Cheval de La Micoque sont encore plus étroites (tabl.13-annexe) (Langlois 2004, tabl.29) que chez Equus caballus germanicus (Combe-Grenal) (fig.16). Leur diamètre mésio-distal (DMD) est toutefois comparable à ceux des Equus mosbachensis. 
Figure $16-$ M1-2 inférieures de différents gisements. Figure 16 - Lower M1-2 from various sites.

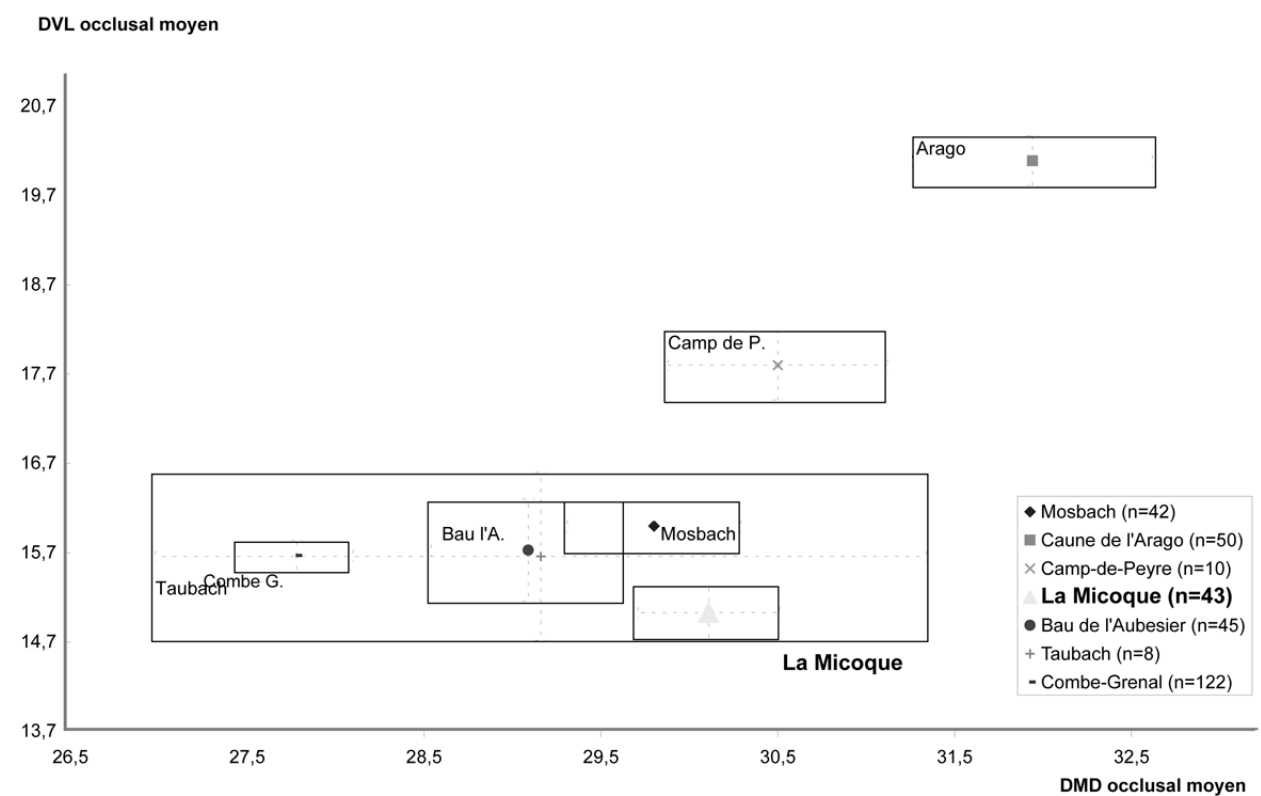

Diamètres mésio-distal et vestibulo-lingual moyens occlusaux et leur intervalle de confiance à $95 \%$. Average mesio-distal and vestibulo-lingual occlusal diameters and their reliable intervals up to $95 \%$.

\section{M3 inférieures}

Les troisièmes molaires inférieures du Cheval de La Micoque sont relativement étroites, comme les autres jugales inférieures (tabl. 14-annexe) (Langlois 2004, tabl. 30). En cela il est proche des Chevaux du Bau de l'Aubesier et de Combe-Grenal (fig.17). Le diamètre mésio-distal (DMD) est intermédiaire entre ceux des chevaux de Mosbach et de la Caune de l'Arago d'une part et de Jaurens et Camiac d'autre part. 
Figure $17-M 3$ inférieures de différents gisements. Figure 17 - Lower M3 from various sites.

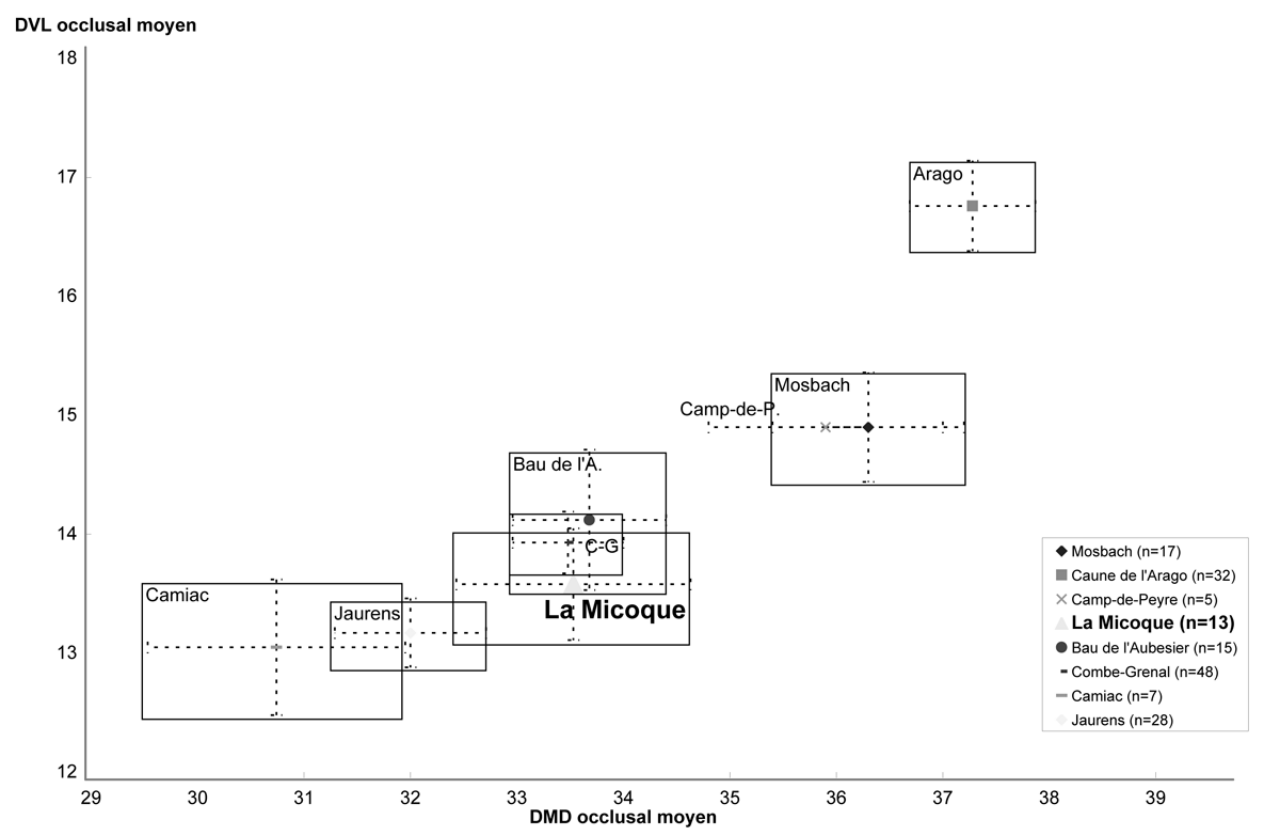

Diamètres mésio-distal et vestibulo-lingual moyens occlusaux et leur intervalle de confiance à $95 \%$. Average mesio-distal and vestibulo-lingual occlusal diameters and their reliable intervals up to $95 \%$.

Le Cheval de La Micoque appartient bien au groupe des Chevaux de type mosbachensis avec cependant quelques particularités, à savoir : des jugales supérieures et inférieures assez longues (diamètre mésio-distal grand) mais le plus souvent très peu épaisses (diamètre vestibulo-lingual faible); un protocône court sur les molaires. Le Cheval de La Micoque se rapproche ainsi des formes plus récentes de Biache-Saint-Vaast, du Bau de l'Aubesier voire même d'Equus caballus germanicus.

\subsection{2 - Squelette crânien et post-crânien : caractéristiques morphologiques}

Les fragments crâniens déterminables sont peu nombreux, seuls quelques fragments de rochers ont été identifiés. Deux fragments relativement complets ont permis une étude morphologique détaillée (Mic88, N27-1135, c. Eb ; Mic84, P28-66, c.L2/3). L'étude de ces fragments a été rendue possible grâce à l'aide précieuse de J.-L. Guadelli (en préparation). Ces deux rochers ont une morphologie relativement comparable à celle des rochers des Equus caballus. Toutefois, quelques particularités également notées sur la portion pétreuse du temporal chez le Cheval de Camp-de-Peyre sont à relever :

1. caudalement au méat auditif, on trouve une faible dépression semi-circulaire limitée du côté rostro-dorsal par un court relief haut et étroit d'orientation ventro-rostral/dorso-caudal, contrairement à ce qu'on peut voir chez Equus caballus (fig.18-a). L'orifice de l'aqueduc du vestibule (Apertura externa aquaeductus vestibuli) est étroit et débouche à une grande distance du bord de la face médiale (fig.18-b), or chez Equus caballus, il débouche près de ce bord ;

2. la moitié dorsale du bord caudal de la face médiale est à peu près rectiligne et peu oblique et se développe du côté médial (fig.18-c), chez Equus caballus, elle est plus ou moins irrégulière et très oblique et à tendance à se développer du côté caudal ;

3. la face caudale (Facies caudalis partis petrosae), qui est soudée à l'os occipital, est irrégulière, vaste, peu oblique en vue ventrale et se développe du côté médial (fig.19). Chez Equus 
caballus, elle est vaste, plus ou moins plane et oblique en vue ventrale et se développe du côté médio-caudal voire caudale.

Figure 18 - La Micoque. Rocher droit, face médiale (N27/88, c. Eb, $\left.n^{\circ} 1135\right)$.

Figure 18 - La Micoque. Right petrosal bone, medial side (N27/88, level Eb, $\left.n^{\circ} 1135\right)$.

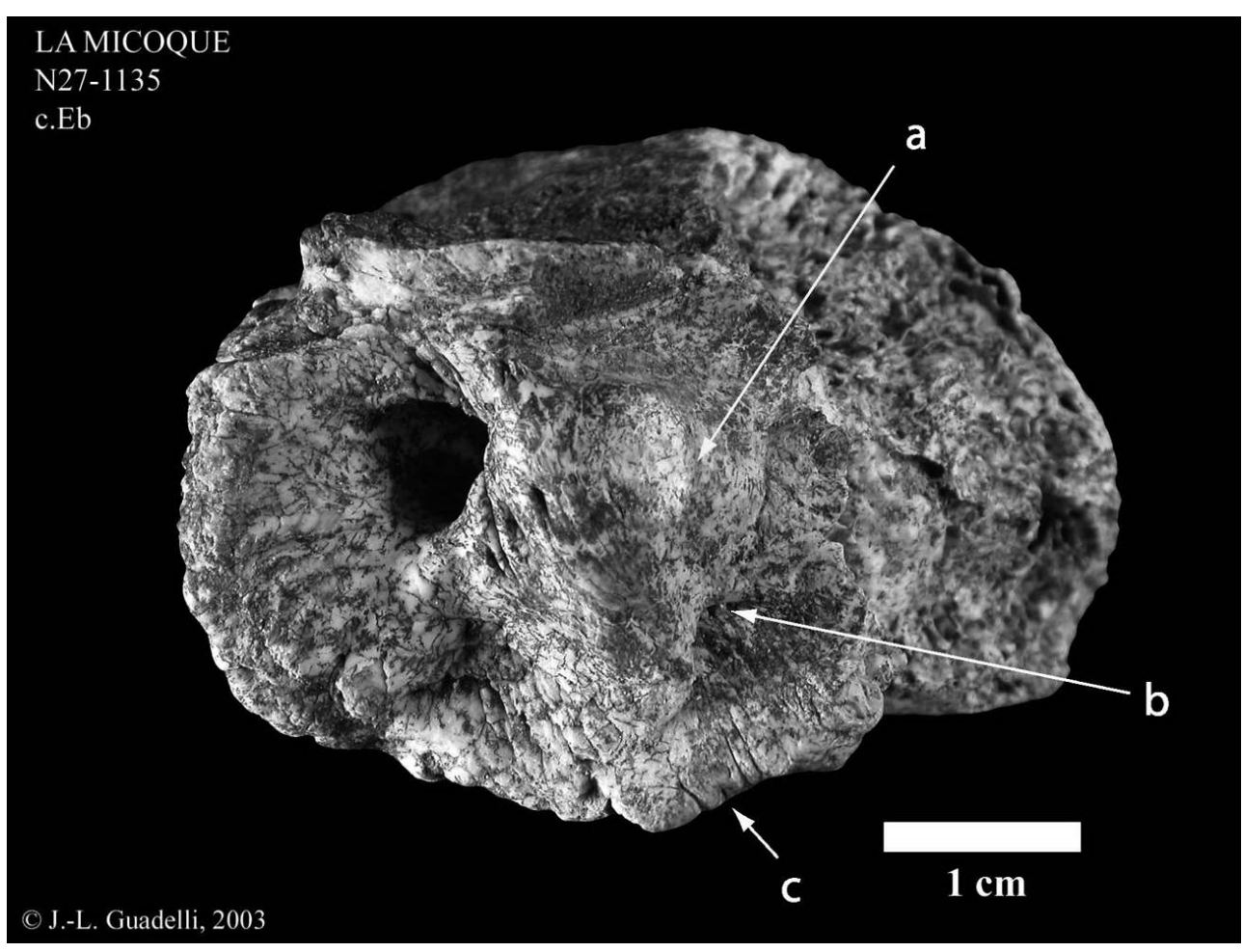

Figure 19 - La Micoque. Rocher droit, face caudale (P28/84, c. L2/3, $\left.n^{\circ} 66\right)$.

Figure 19 - La Micoque. Right petrosal bone, caudal view (P28/84, level L2/3, $\left.n^{\circ} 66\right)$.

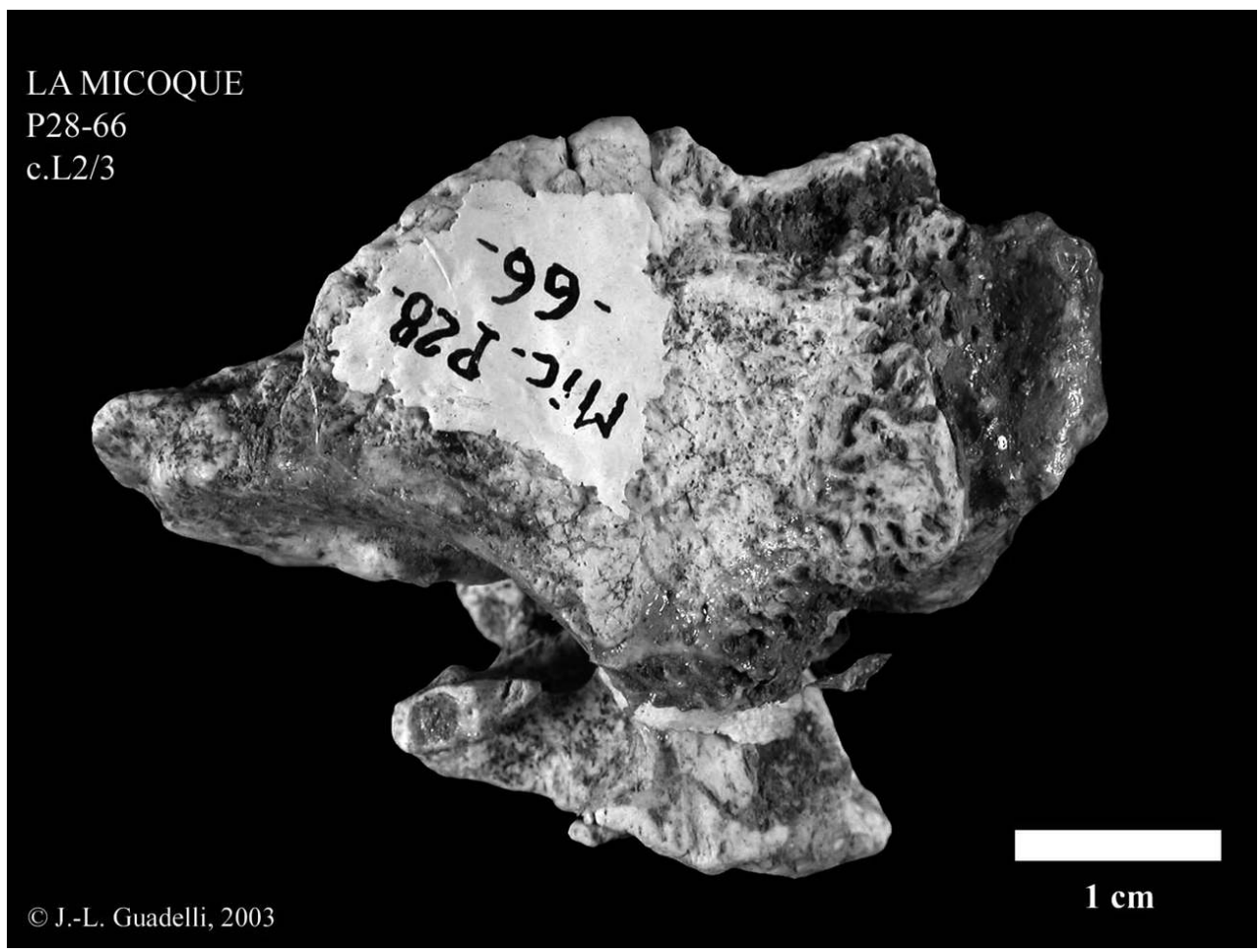



médiale de l'extrémité proximale, une gouttière tendineuse qui n'est plus visible sur ce même os chez les chevaux plus récents (fig.20). Cette caractéristique est visible chez le Chevaux de Camp-de-Peyre (Guadelli et Prat 1995). Cette coulisse est surmontée d'une tubérosité bicipitale très large et massive, ceci est visible sur plusieurs fragments retrouvés à La Micoque. Ces différents caractères sont assez proches de ceux qu'on peut voir chez Equus mosbachensis campdepeyri. Ils évoquent la présence de tendons relativement plus robustes et plus puissants que chez des chevaux plus récents, nous pouvons donc dire qu'il s'agit de caractères archaïques.

Figure 20 - La Micoque. Extrémité proximale d'un radius gauche présentant une coulisse tendineuse bien marquée, face médiale (N24/93, c. E., $\mathrm{n}^{\circ} 848$ ).

Figure 20 - La Micoque. Proximal extremity of a left radius showing a pronounced tendinous groove, medial side (N24/93, level $\left.E, n^{\circ} 848\right)$.

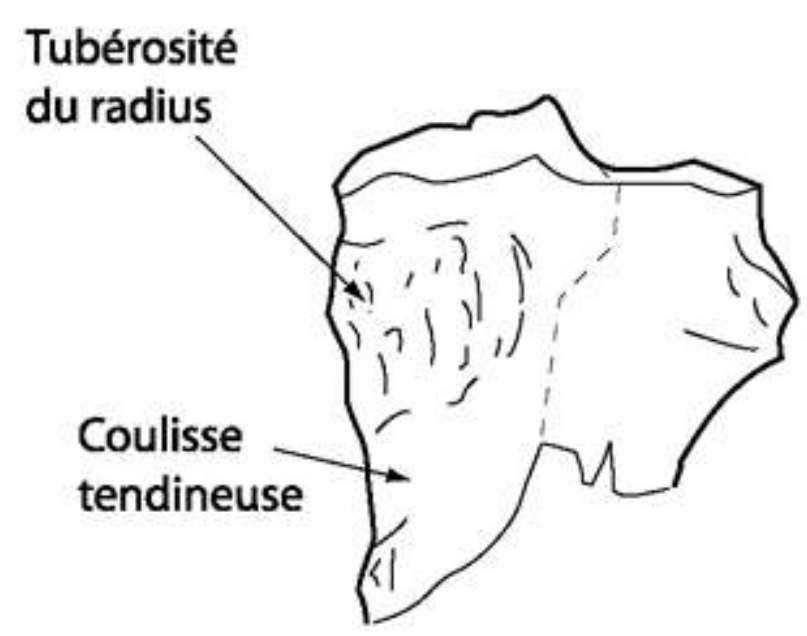

Certains os du carpe ainsi que des os du métapode peuvent présenter des facettes articulaires supplémentaires. Ce phénomène est visible sur les trapézoïdes et les grands os (capitatums) (fig.21, 22). D’après M.-F. Bonifay (1980), l'apparition de ces petites facettes est la conséquence de la mise en place de la monodactylie. Il s'agirait de caractères évolués.

De plus, certains unciformes présentent une courbure assez marquée de leur "processus palmaire ", caractères dont nous devons rechercher la signification et la fréquence sur les chevaux de type mosbachensis (fig.23). 
Figure $21 \mathrm{a}$-La Micoque. Capitatum et trapézoïde droits appartenant à un même individu, face distale $\left(023 / 93\right.$, c. ?, $\left.n^{\circ} 433\right)$.

Figure 21a - La Micoque. Right capitatum and trapezoide belonging to a same individual, distal side (023/93, level ?, $\left.n^{\circ} 433\right)$.

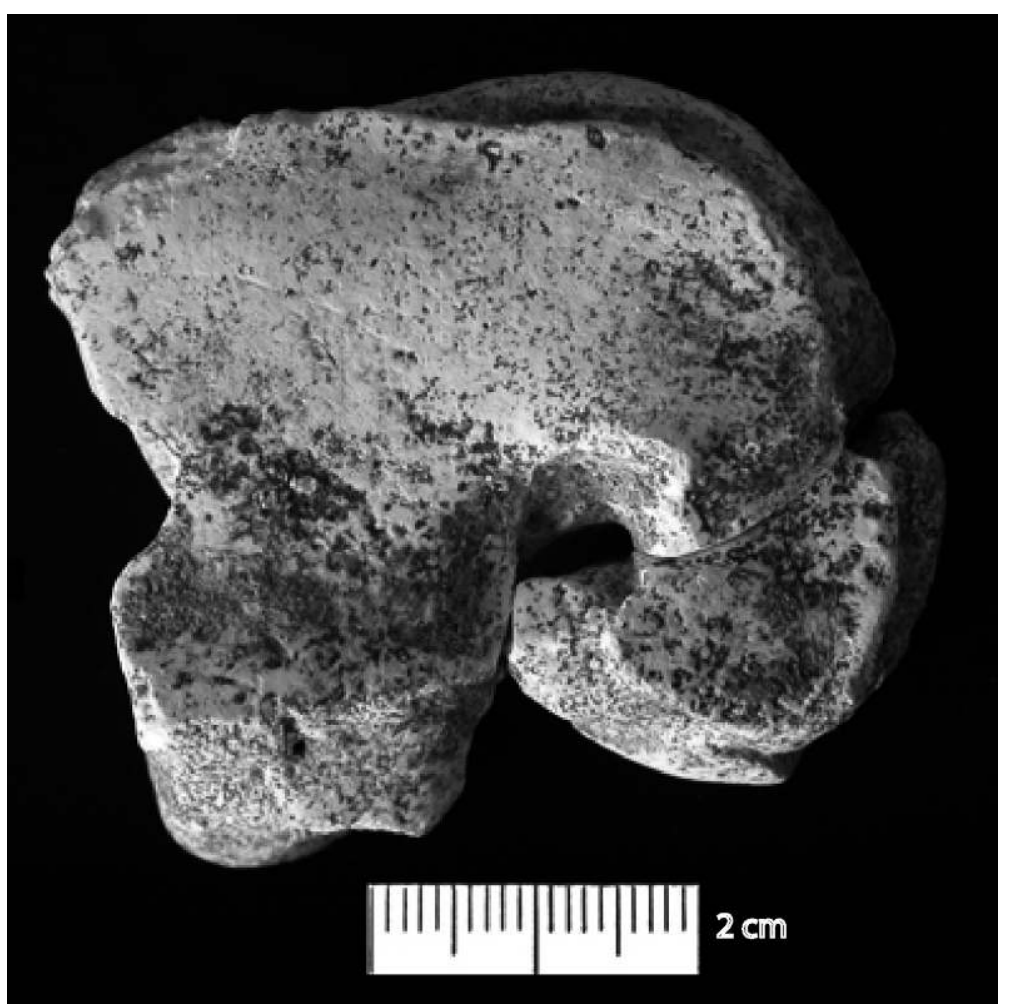


Figure 21b - La Micoque. Capitatum droit (même os que a) avec deux facettes articulaires pour le trapézoïde, face palmaire.

Figure 21b - La Micoque. Right capitatum with two articular facets for the trapezoide, palmar side.

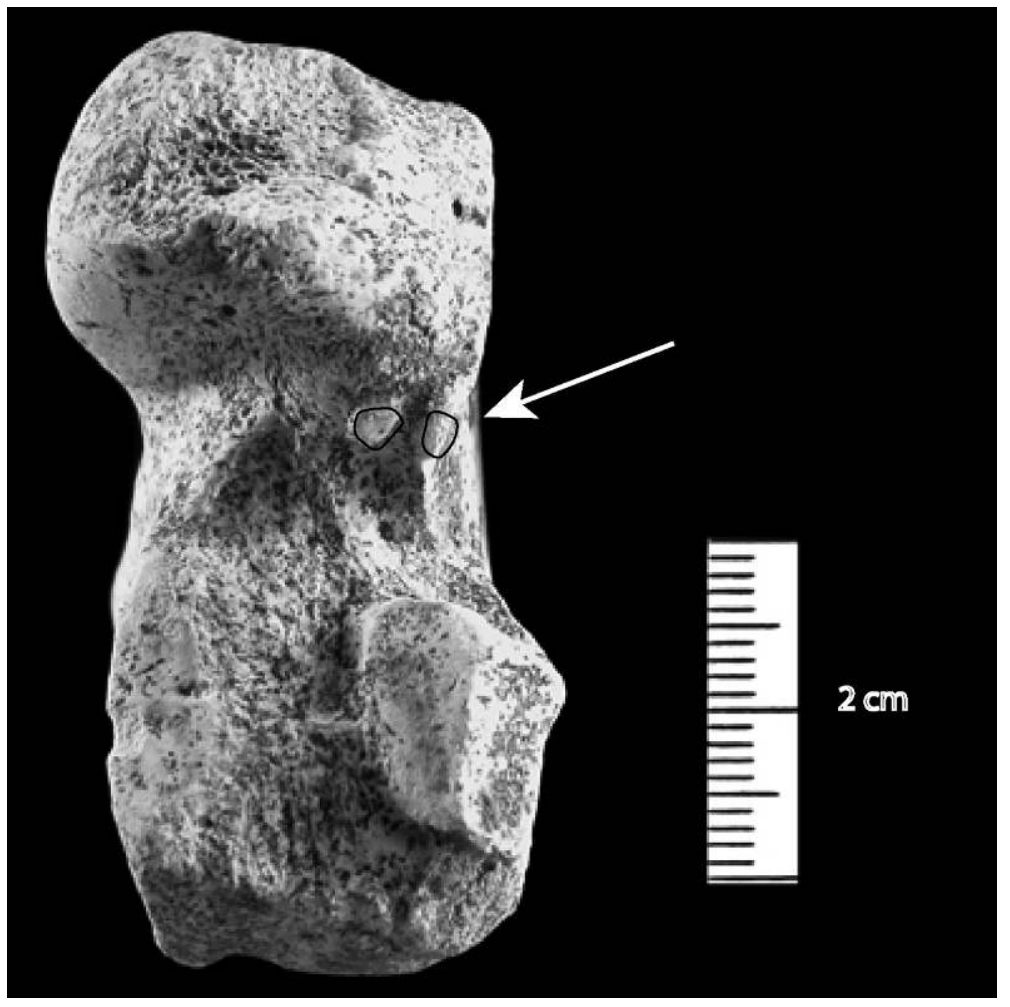


Figure 21c - La Micoque. Trapézoïde droit (même os que a) avec deux petites facettes pour le capitatum et une plus grande pour le métacarpien III, face latérale.

Figure 21c - La Micoque. Right trapezoide with two small facets for the capitatum and one larger facet for the metacarpal III, lateral side.

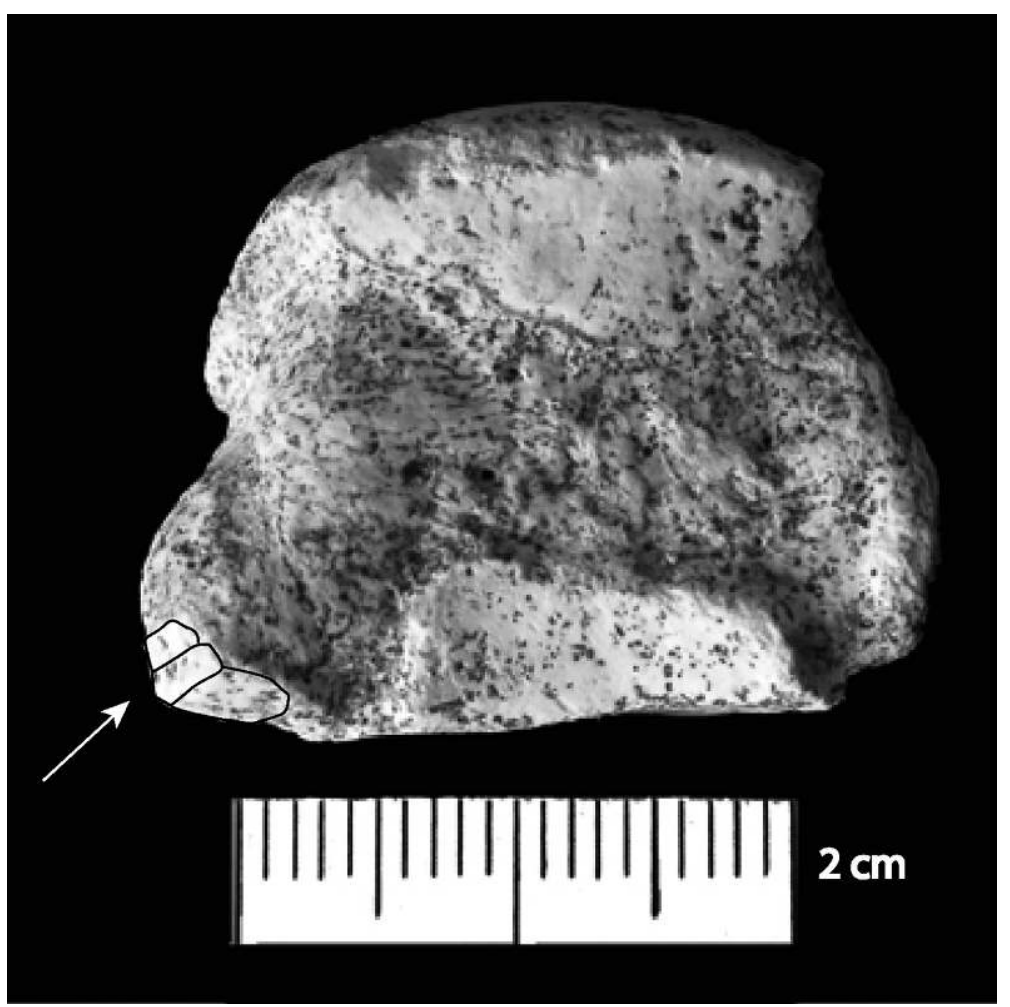


Figure 22 - La Micoque. Trapézoïde droit (Q28/ ?, c. J) avec une seule facette articulaire pour le capitatum et aucune pour le métacarpien III, face latérale.

Figure 22 - La Micoque. Right trapezoide (Q28/?, level J) with only one articular facet for the capitatum and none for the metacarpal III, lateral side.

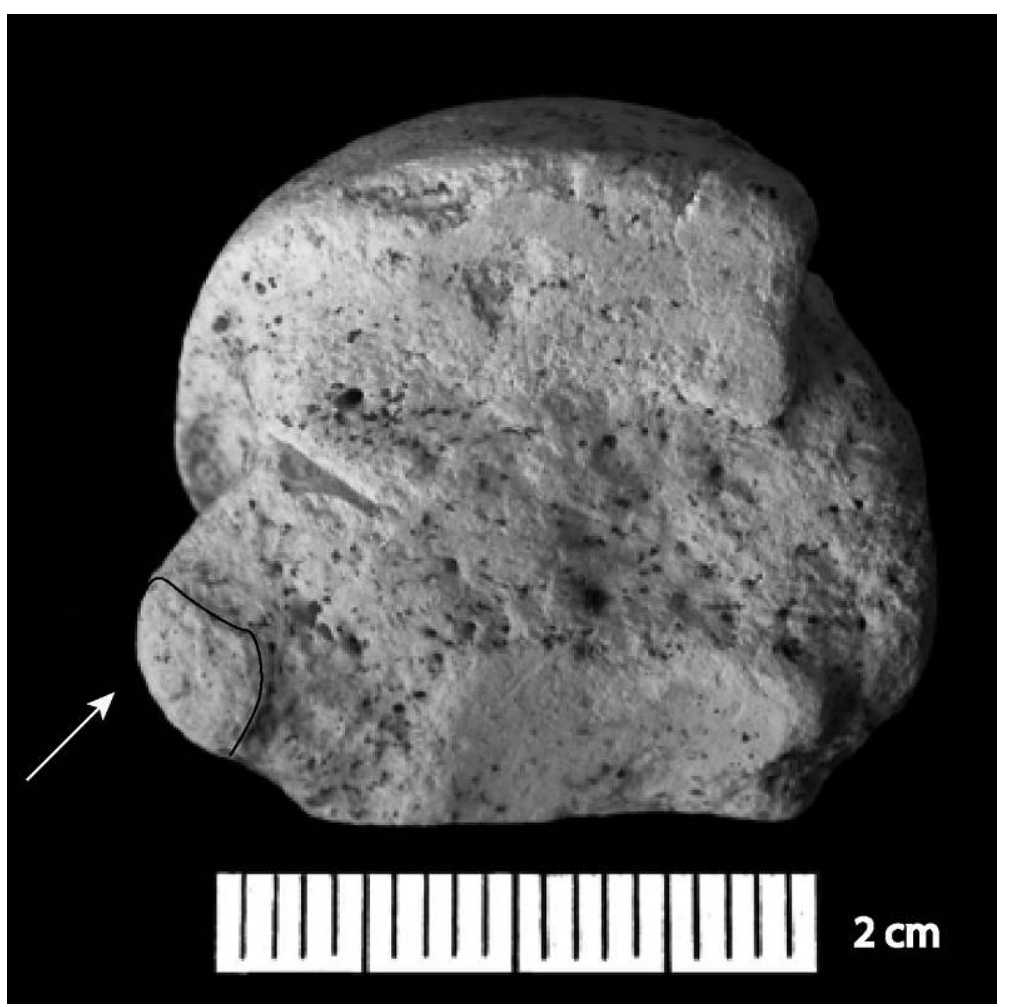

Figure 23 - La Micoque. Unciformes dont le « processus palmaire » est plus ou moins courbe, face latérale : à gauche, deux unciformes gauches (P28/91, c. J et $\left.028 / 89, \mathrm{c} . \mathrm{J}, \mathrm{n}^{\circ} 1713\right)$ et à droite, deux unciformes droits (Q28/ ?, c. J et 028/86, c. ?, $\left.n^{\circ} 318\right)$.

Figure 23 - La Micoque. Unciformes, whose "palmar processus" is more or less curved, lateral side: on the left, two left unciformes ( $P 28 / 91$, level $\mathrm{J}$ and $028 / 89$, level $\left.\mathrm{J}, n^{\circ} 1713\right)$ and on the right, two right unciformes (Q28/?, level $J$ and $028 / 86$, level ?, $\left.n^{\circ} 318\right)$.

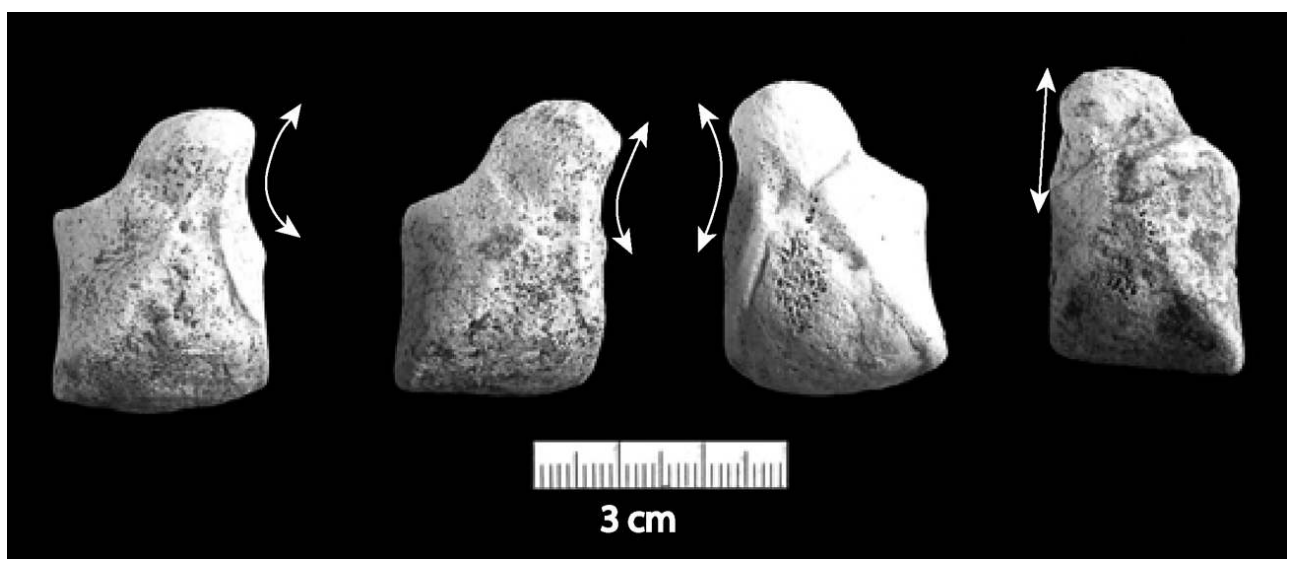

50 Les premières phalanges antérieures et postérieures possèdent une face palmaire très peu proéminente comme c'est le cas avec les Chevaux de Camp-de-Peyre. 


\subsection{3 - Squelette post-crânien : caractéristiques biométriques}

51 En ce qui concerne les os du squelette post-crânien, l'étude biométrique montre que les restes de chevaux trouvés à La Micoque appartiennent à des individus faisant partie des grands Chevaux de type mosbachensis.

52 Les fragments de vertèbres (une caudale, une thoracique et une lombaire de juvénile) n'ont fourni aucune donnée biométrique et morphologique permettant de faire la distinction entre le Cheval de La Micoque et les autres chevaux.

53 Les scapulas sont représentées par cinq cavités glénoïdes (tabl. 15-annexe) (Langlois 2004, tabl. 35 et 36), leur diamètre antéro-postérieur (DAP) n'est pas différent de celui des chevaux des gisements de la Caune de l'Arago et de Lunel-Viel. Toutefois, le diamètre antéro-postérieur des cavités glénoïdes du Cheval de La Micoque est relativement grand par rapport à Equus caballus gallicus et les chevaux sauvages actuels : Equus przewalskii.

54 Les humérus sont fragmentés c'est pourquoi peu de mesures ont été prises (tabl.16annexe) (Langlois 2004, tabl. 38 et 39). Le diamètre transversal (DT) de l'articulation distale et le diamètre antéro-postérieur (DAP) minimum de la trochlée sont comparables aux mêmes mesures chez les chevaux de La Caune de l'Arago et de l'Abri Suard.

55 Le radio-ulna possède des extrémités dont les mesures (tabl.17-annexe) sont comparables à celles du même os chez Equus caballus germanicus mais les diamètres transversaux des condyles distaux sont très proches de ce qu'on peut observer chez Equus mosbachensis du Bau de l'Aubesier et de Biache-Saint-Vaast (Langlois 2004, Annexe 17).

56 Les os du carpe ont, pour la plupart, des dimensions (tabl. 18 à 20-annexe) se rapprochant des mêmes valeurs observables chez le Cheval de l'Arago et Equus caballus piveteaui (Langlois 2004, tabl. 42 à 54).

57 Le deuxième métacarpien du Cheval de La Micoque montre un diamètre antéropostérieur (DAP) plus important que ceux des "grands » chevaux des sites de BiacheSaint-Vaast, Bau de l'Aubesier et Lunel-Viel (tabl. 21-annexe) (Langlois 2004, Annexe 18).

58 Pour le quatrième métacarpien, aucune différence significative n'est visible entre les Chevaux de La Micoque (tabl. 21-annexe) (Langlois 2004, tabl. 56 et Annexe 18) et les chevaux de référence.

59 A partir des dimensions des extrémités proximale et distale du troisième métacarpien (tabl. 22-annexe), nous pouvons faire la distinction entre plusieurs groupes. Pour les extrémités proximales (tabl.22-annexe, fig.24), trois groupes se démarquent: le premier groupe rassemble les chevaux dont les extrémités sont très petites comme Equus caballus gallicus de Saint-Germain-la-Rivière et Solutré; le deuxième groupe est composé des chevaux d'Achenheim et de Caune de l'Arago, appartenant à l'espèce mosbachensis, qui présentent des extrémités proximales très grandes; le troisième regroupe les chevaux de La Micoque, de Camp-de-Peyre et de Biache-Saint-Vaast mais également Equus caballus germanicus de Combe-Grenal qui présentent des dimensions intermédiaires. Pour les extrémités distales (tabl. 22-annexe, fig.25), il est possible de distinguer un premier groupe composé d'Equus caballus germanicus du site de CombeGrenal et d'Equus caballus gallicus des sites de Saint-Germain-la-Rivière, Solutré et 
Camiac. Ces Équidés présentent des extrémités distales relativement petites. Les grands chevaux, quant à eux, constituent le deuxième groupe, avec toutefois, des chevaux à extrémités distales très grandes comme les chevaux de Caune de l'Arago et d'Achenheim et des chevaux à extrémités moins robustes comme les chevaux de LunelViel et de La Micoque ou encore comme Equus caballus germanicus de Pair-non-Pair.

Figure 24 - Dimensions des extrémités distales des métacarpiens III des différents chevaux de référence.

Figure 24 - Strength of proximal extremities of metacarpals III of different horses.

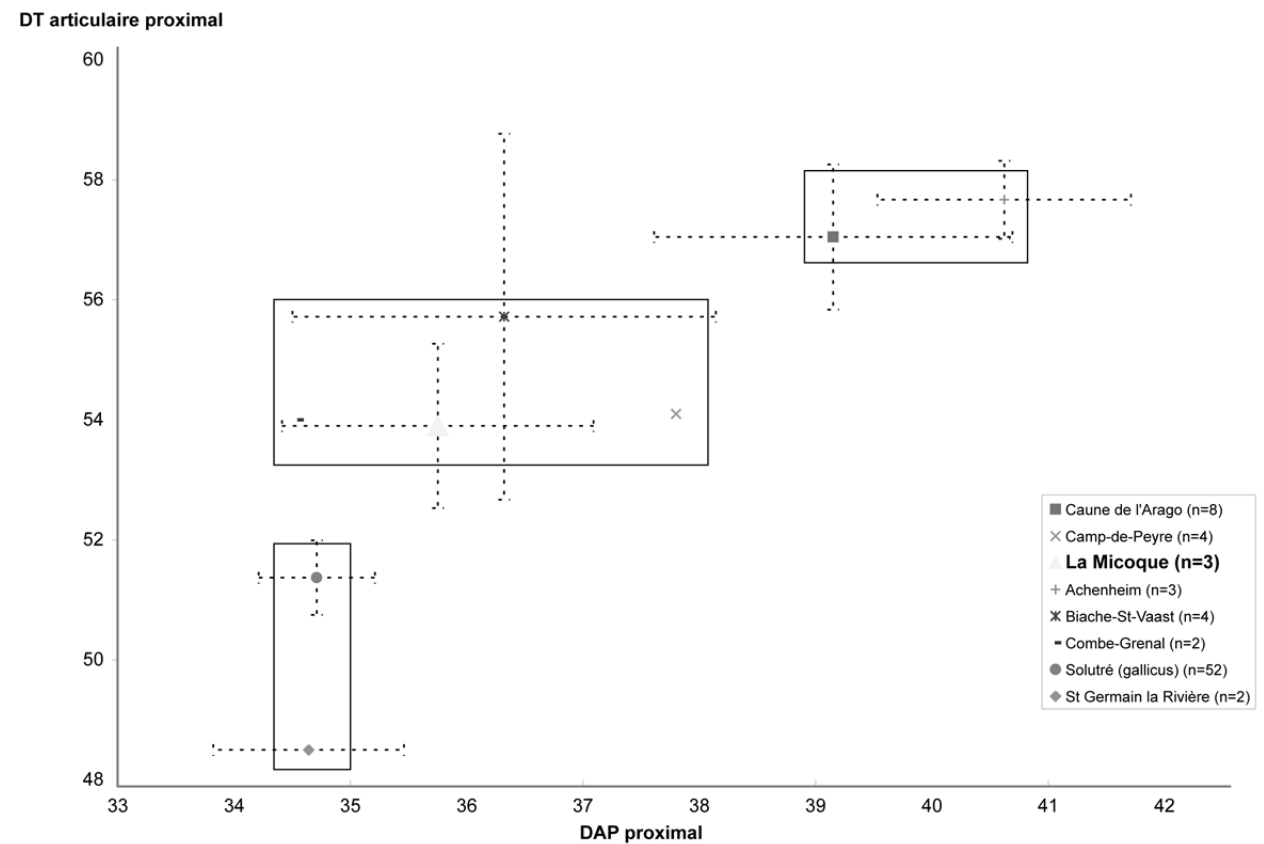


Figure 25 - Dimensions de l'extrémité distale des métatarsiens III des différents chevaux de référence.

Figure 25 - Measurements of distal extremities of metacarpals III of different horses.

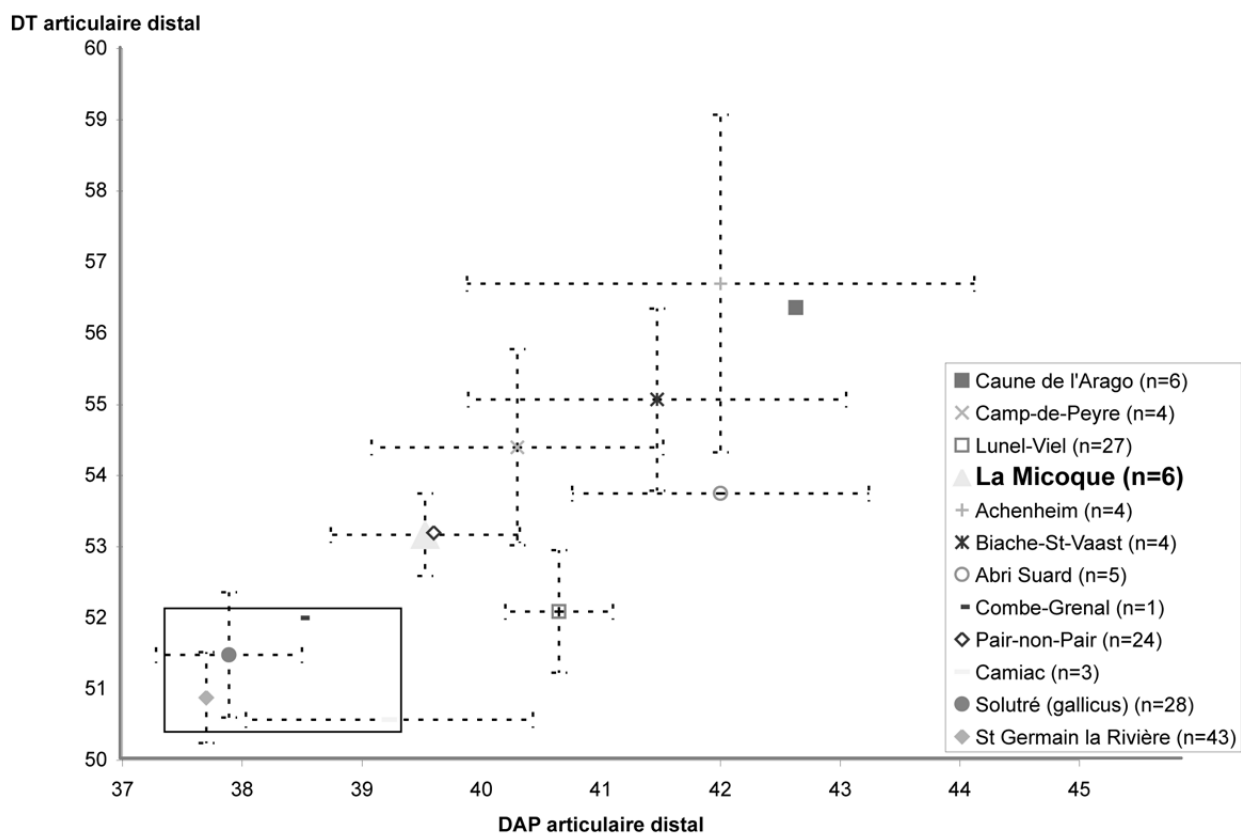

60 L'étude biométrique des phalanges (tabl.23-annexe) ne permet pas de faire une distinction entre les différents chevaux de référence (Langlois 2004, Annexes 21, 22). Les os coxaux ne sont représentés que par quelques fragments qui n'ont pas pu être mesurés. De plus, il existe très peu de références métriques relatives aux différents groupes d'Équidés.

Seuls quatre fragments de têtes fémorales ont été retrouvés à La Micoque (tabl. 24annexe). Le diamètre antéro-postérieur (DAP) de cette articulation semble relativement proche des mêmes valeurs mesurées pour les chevaux de la Caune de l'Arago (Langlois 2004, chapitre 2-IV.2.2.).

La patella n'est représentée que par un seul exemplaire. Ses dimensions (tabl.25annexe) correspondent aux valeurs les plus faibles des patella des chevaux de Caune de l'Arago (Langlois 2004, tabl.59). Les extrémités distales de tibia des Chevaux de La Micoque montrent un diamètre antéro-postérieur (DAP) plus faible (tabl.26-annexe) que chez les chevaux de type mosbachensis des sites de Mosbach, Montoussé et Achenheim mais il est semblable à celui des tibias des chevaux de la Caune de l'Arago et de ceux d'Equus caballus piveteaui (Abri Suard). Pour le diamètre transversal (DT), les valeurs trouvées chez les différents chevaux sont semblables (Langlois 2004, tabl. 60).

Pour les os du tarse, le talus (tabl. 27-annexe) présente un diamètre transversal (DT) de la lèvre interne de la trochlée plus faible chez le Cheval de La Micoque que chez les chevaux du groupe mosbachensis des sites d'Achenheim, Caune de l'Arago, Lunel-Viel (Langlois 2004, Annexe 23). Equus caballus piveteaui, quant à lui, possède un talus de dimensions plus grandes dans son ensemble comparé à celui du Cheval de La Micoque. Le calcaneus, en faible nombre, est assez court (tabl.28-annexe), sa longueur est semblable à la longueur des calcanéums appartenant au Cheval de Przewalski (Gromova 1949b). Il est également étroit comme M.-F. Bonifay le remarque pour l'Équidé de Lunel-Viel (Bonifay 1980). Le diamètre antéro-postérieur (DAP) du bec est faible, 
comparable à ce qui a été décrit pour Equus caballus germanicus ou Equus caballus gallicus (Langlois 2004, Annexe 24).

Les naviculaires trouvés à La Micoque (tabl. 29-annexe) sont à rapprocher des mêmes os des grands chevaux tels que ceux de Caune de l'Arago, ceux de Lunel-Viel et Equus caballus piveteaui de l'Abri Suard. Les naviculaires d'Equus caballus germanicus et Equus caballus gallicus sont plus petits (Langlois 2004, tabl. 64).

Pour les cuboïdes, nous n'avons aucune valeur pour Equus mosbachensis. Cependant leurs diamètres transversaux sont proches des valeurs données pour Equus caballus germanicus et Equus caballus gallicus (tabl. 29-annexe) (Langlois 2004, tabl. 66).

67 Les grands cunéiformes du Cheval de Lunel-Viel et Equus caballus piveteaui sont semblables à ceux découverts à La Micoque (tabl. 30-annexe; Langlois 2004, tabl. 68).

Les petits cunéiformes sont relativement courts (tabl.30-annexe), comme chez Equus caballus gallicus tandis que leurs diamètres transversaux se rapprochent de ceux visibles chez le Cheval de Caune de l'Arago et Equus caballus gallicus de Solutré (Langlois 2004, tabl. 70).

Les métatarsiens accessoires (II et IV) sont représentés par leur extrémité proximale (tabl. 31-annexe). Leurs dimensions sont données à titre comparatif car ils sont trop peu nombreux dans les autres gisements de référence (Langlois 2004, tabl. 72 et 73).

Pour le métatarsien III, l'étude biométrique (tabl. 32-annexe) (Langlois 2004, Annexes 25 et 26) nous permet de distinguer plusieurs groupes parmi les Équidés de référence. On remarque que 3 groupes se distinguent (fig.26): le premier est composé d'Equus caballus gallicus des sites de Camiac, Solutré et St Germain-la-Rivière, l'extrémité distale des métatarsiens III de ces Équidés est petite ; le second rassemble les chevaux des sites d'Achenheim et de Biache-Saint-Vaast attribués à l'espèce mosbachensis mais également Equus caballus piveteaui de l'Abri Suard, ces chevaux possèdent des métatarsiens III dont l'extrémité distale est grande. Entre ces deux groupes, nous individualisons un troisième ensemble comprenant les chevaux de La Micoque, de Caune de l'Arago et de Camp-de-Peyre. L'équidé de Lunel-Viel possède des métatarsiens dont le diamètre transversal (DT) de l'extrémité distale est comparable à celui des Equus caballus gallicus mais le diamètre antéro-postérieur (DAP) est proche de celui des chevaux de La Micoque, de Camp-de-Peyre et de la Caune de l'Arago. Fautes de données, la comparaison avec des chevaux plus anciens tels que le Cheval de Mosbach n'a pu être faite. En définitive il apparaît, comme pour les métacarpiens III, que le Cheval de La Micoque possède des métatarsiens III dont les dimensions de l'extrémité distale sont comparables à celles des chevaux de type mosbachensis. 
Figure 26 - Dimensions de l'extrémité distale des métatarsiens III des différents chevaux de référence.

Figure 26 - Measurements of distal extremity of metatarsals III of different horses.

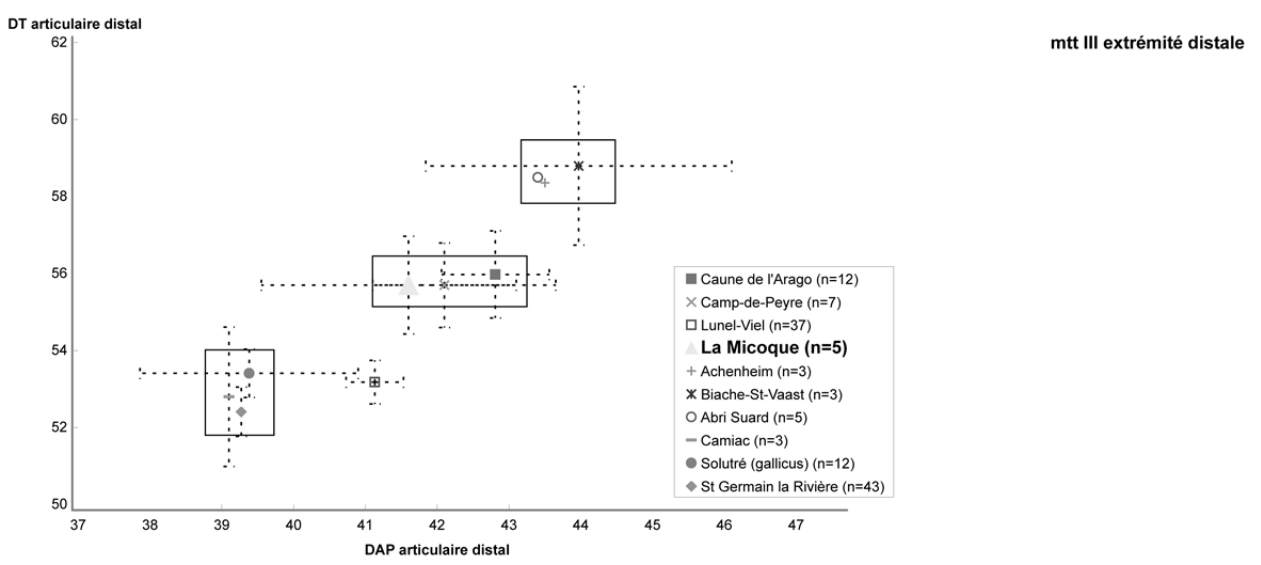

71 Nous venons de voir que les chevaux des différents sites peuvent se répartir en fonction des dimensions de l'extrémité distale de leurs os canons. Cette répartition s'avère identique si l'on considère le troisième métacarpien ou le troisième métatarsien. Il est important de noter que les chevaux appartenant aux espèces germanicus et gallicus possèdent des os canons dont l'extrémité distale est petite, il en est de même pour le Cheval de Lunel-Viel. Chez les Chevaux des sites d'Achenheim et de Biache-Saint-Vaast, ces mêmes os présentent des extrémités distales grandes voire très grandes. Chez le Cheval de l'Arago, le troisième métacarpien possède des extrémités proximale et distale relativement grandes alors que le troisième métatarsien montre une extrémité distale plutôt petite. Le Cheval de La Micoque, quant à lui, possède des os canons dont les extrémités distale et proximale ont des dimensions plus importantes que chez les chevaux de l'espèce Equus caballus mais plus petites que chez les chevaux de BiacheSaint-Vaast et Achenheim.

Enfin, les phalanges postérieures ne présentent pas de mensurations significativement différentes des phalanges des autres Équidés (tabl. 33-annexe) (Langlois 2004, Annexes 27 et 28). Les deux premières phalanges, malgré un échantillon restreint, paraissent toutefois plus graciles que celles des chevaux de Biache-Saint-Vaast et de Mosbach.

73 L'étude biométrique des os du squelette montre que le Cheval de La Micoque appartient sans aucun doute à l'espèce Equus mosbachensis. Cependant, certains os possèdent des dimensions qui peuvent rapprocher le Cheval de La Micoque de chevaux tels que Equus caballus piveteaui (dimensions de l'extrémité distale des humérus, des os du carpe et de quelques os du tarse) ainsi que Equus caballus germanicus (dimensions des extrémités des radio-ulnas, de l'extrémité distale des métacarpiens III, ou de quelques os du tarse). Nous pouvons noter également que les os canons présentent des extrémités dont la taille est intermédiaire entre celle rencontrée chez les grands chevaux appartenant à l'espèce mosbachensis et celles des chevaux plus récents comme Equus caballus germanicus et Equus caballus gallicus.

\section{3 - Attribution taxonomique du cheval de La Micoque}

74 A ce stade des études biométriques et morphologiques sur le matériel osseux et dentaire, il est possible de confirmer l'attribution du Cheval de La Micoque à l'espèce 
Equus mosbachensis. Ce Cheval paraît être à un stade d'évolution intermédiaire entre d'une part les Equus mosbachensis trouvés à Mosbach, Camp-de-Peyre, Lunel-Viel et Caune de l'Arago et d'autre part Equus caballus piveteaui, plus proche cependant d'un point de vue évolutif du second que du premier. Cette position ne va pas à l'encontre des âges radiométriques : le stade isotopique 10 (Falguères et al. 1997) et géologiques (Texier et Bertran 1993) proposés pour le gisement de La Micoque. L'attribution taxonomique du Cheval de La Micoque confirme les études précédentes qui rapprochaient ce Cheval de l'espèce Equus mosbachensis du Pléistocène moyen. L'étude approfondie des restes de ce Cheval nous permet, en effet, de confirmer que cet Équidé correspond aux chevaux de type mosbachensis mais il présente suffisamment de particularités pour justifier, nous semble-t-il, la création d'une sous-espèce. Celle-ci, que nous proposons d'appeler Equus mosbachensis micoquii, possède des caractères biométriques et morphologiques particuliers au sein de la lignée des Chevaux du Pléistocène moyen.

Equusmosbachensismicoquii nov. ssp.

Holotype : série inférieure droite : P2-P3-P4-M1 notée Mic 85, O 25, c. L2/3, nº 479 (fig. 27)

Paratype : tout le matériel dentaire et osseux attribué au Cheval issu des fouilles de A.

Debénath et J.-Ph. Rigaud entre 1983 et 1996

Localité-type : La Micoque aux Eyzies-de-Tayac

Niveau type : Ensemble moyen, ensemble stratigraphique F4 (couches D, F, G, I à K) et ensemble stratigraphique DP3 (couches E, H, J et L).

Diagnose :

1. Denture :

Morphologie typiquement caballine (sensu lato), à protocône relativement court sur les M1/-2/ et les M3/. Les M1/-2/ et toutes les jugales inférieures ont un diamètre vestibulolingual faible voire très faible.

2. Squelette post-crânien :

Région proximale du radius : le bord dorsal de la cavité glénoïdale médiale est oblique ; la coulisse tendineuse du muscle brachial antérieur, bien marquée, est surmontée d'une tubérosité bicipitale très large et massive.

Os du carpe: Trapézoïde : il présente 1 ou 2 facettes articulaires supplémentaires pour le grand os et/ou le métacarpien III. Grand os: il possède une facette articulaire supplémentaire pour le trapézoïde. L'unciforme présente un processus palmaire très courbe. Métacarpien III : la facette articulaire répondant au trapézoïde est très réduite, celle répondant à l'unciforme est vaste ; l'angulation entre les facettes articulaires correspondant à l'unciforme et au grand os est peu marquée. Insertion musculaire latérale et médiale au niveau de l'extrémité distale très marquée et saillante.

Os du tarse : le calcanéum est relativement court.

Métatarsien III : la facette articulaire correspondant au cuboïde est très petite.

Phalanges antérieures et postérieures : insertion ligamenteuse très peu proéminente sur les faces palmaires et plantaires. 
Figure 27a - Equus mosbachensis micoquii nov.ssp. Holotype : Fragment de mandibule inférieure droite avec $P / 2, P / 3, P / 4$ et $M / 1$ (Mic 85, 025, couche $L 2 / 3, n^{\circ} 479$ )

Figure 27a - Equus mosbachensis micoquii nov. ssp. Holotype : Fragment of right lower mandible with $P / 2, P / 3, P / 4$ and $M / 1$ (Mic 85, 025, level L2/3, $n^{\circ} 479$ )

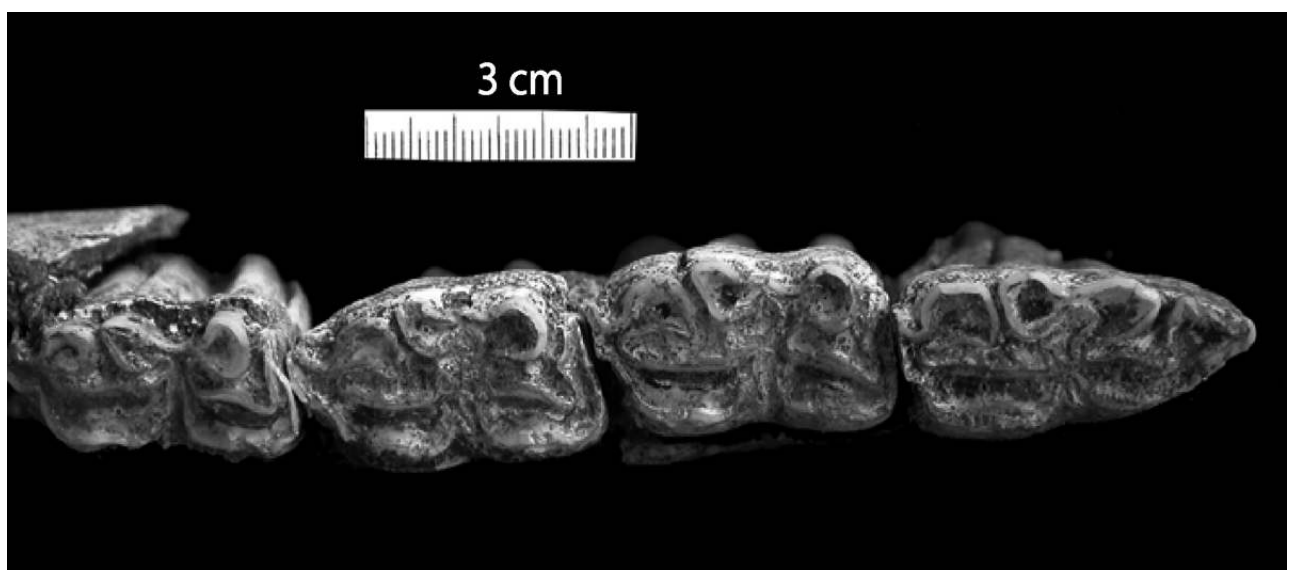

Vue occlusale de la série (P2 à M1 de droite à gauche). Occlusal view of the series (P2 to M1 from right to left)

Figure 27b - Equus mosbachensis micoquii nov.ssp. Holotype : Fragment de mandibule inférieure droite avec $\mathrm{P} / 2, \mathrm{P} / 3, \mathrm{P} / 4$ et $\mathrm{M} / 1$ (Mic 85, 025, couche $\mathrm{L} 2 / 3, \mathrm{n}^{\circ}$ 479)

Figure 27b - Equus mosbachensis micoquii nov. ssp. Holotype : Fragment of right lower mandible with $P / 2, P / 3, P / 4$ and $M / 1$ (Mic 85, 025, level $L 2 / 3, n^{\circ} 479$ )

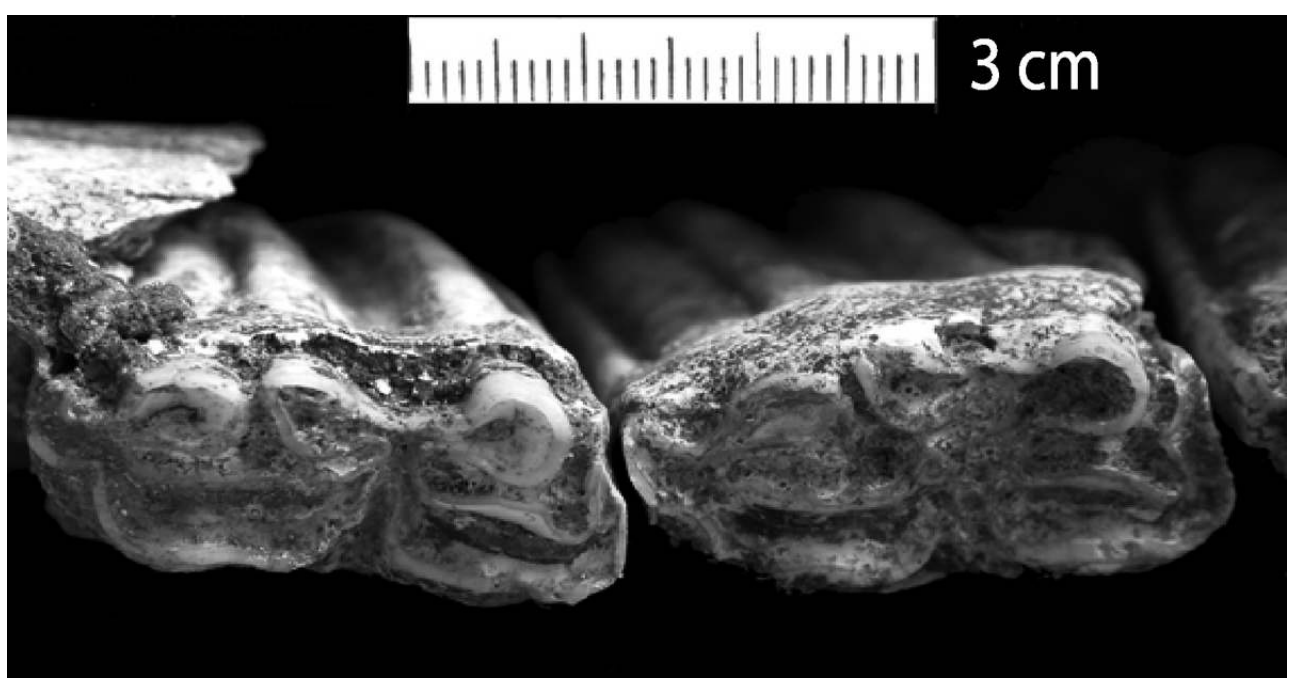

Vue occlusale de P4 et M1.

Occlusal view of $P 4$ and $M 7$. 
Figure 27c - Equus mosbachensis micoquii nov.ssp. Holotype : Fragment de mandibule inférieure droite avec $P / 2, P / 3, P / 4$ et $M / 1$ (Mic 85, 025, couche $L 2 / 3, n^{\circ} 479$ )

Figure 27c - Equus mosbachensis micoquii nov. ssp. Holotype : Fragment of right lower mandible with $P / 2, P / 3, P / 4$ and $M / 1$ (Mic 85, 025, level L2/3, $n^{\circ} 479$ )

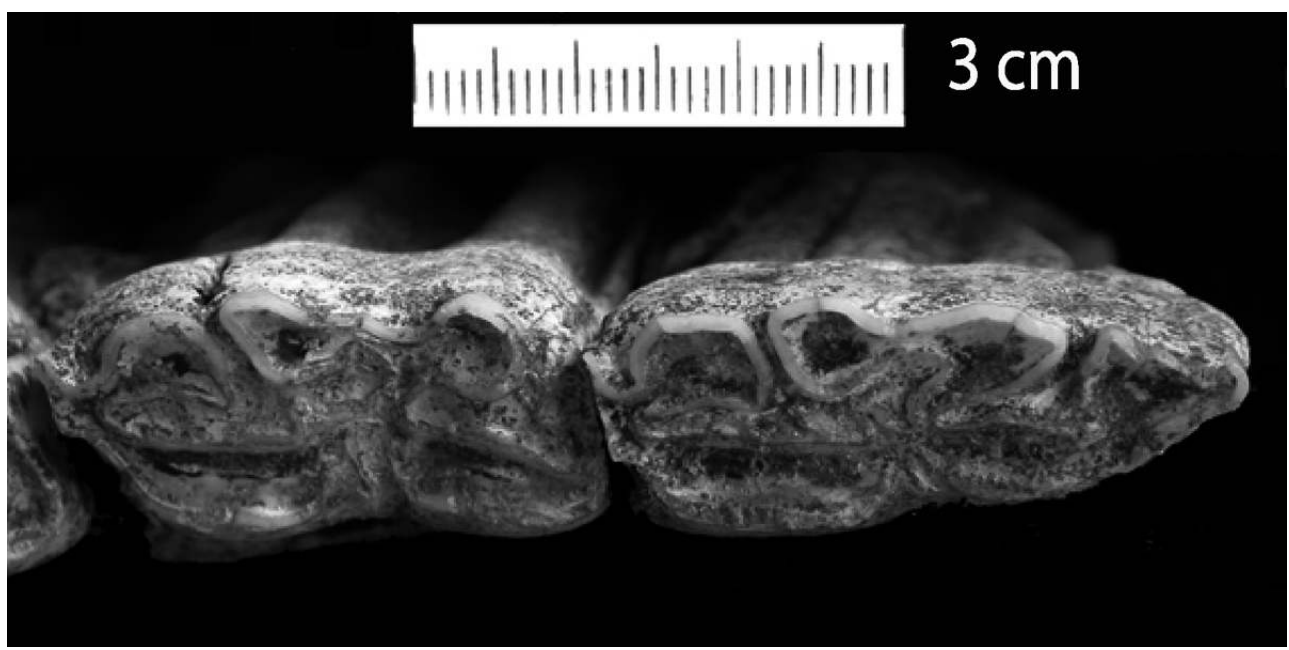

Vue occlusale de P3 et P2.Occlusal view of P3 and P2.

\section{4 - Biostratigraphie : position du gisement de La Micoque par rapport aux autres sites du Pléistocène moyen d'Aquitaine}

77 Le Cheval est un taxon bien représenté dans les gisements archéologiques depuis le milieu du Pléistocène inférieur. De plus, la morphologie osseuse et dentaire a permis aux chercheurs de mettre en évidence au moins une lignée évolutive conduisant du Cheval de Mosbach (datant du Pléistocène inférieur) au petit Cheval de Solutré (Equus caballus arcelini, datant de la fin du Pléistocène supérieur). Le genre Equus est donc de première importance pour la datation relative des sites dans lesquels il est présent.

Au cours de nos études morphologiques et biométriques, il est apparu que de nombreux caractères du Cheval de La Micoque correspondaient à ceux d' Equus mosbachensis, à savoir des jugales supérieures dont l'indice protoconique ne cesse d'augmenter de la P2 à la M3, des jugales inférieures relativement étroites (DVL peu élevé), une gouttière tendineuse présente sur la face médiale de l'extrémité proximale du radio-ulna; une extrémité distale des métacarpiens et métatarsiens principaux dont les tubercules susarticulaires sont assez développés. Quelques particularités, notamment pour les dents et les os courts, suggèrent qu'il se positionne très près des chevaux de Biache-SaintVaast et du Bau de l'Aubesier voire même d'Equus caballus germanicus. Parmi les caractères considérés comme " évolués ", rappelons notamment :

1. la petite taille des $\mathrm{P} 2$ et des $\mathrm{P} 3-4$ supérieures;

2. la faible épaisseur des premières et deuxièmes molaires supérieures et inférieures;

3. le faible développement relatif du protocône des M1-2 et M3 supérieures.

Si l'on considère ces caractères comme des caractères évolutifs, les caballins se seraient ainsi succédés, du plus ancien au plus récent (tabl. 34-annexe). 
80 espèce. Comme nous l'avons dit précédemment, de nombreux auteurs font d'Equus mosbachensis une espèce distincte. D'après les études morphologiques et biométriques, notamment des jugales supérieures, nous adhérons totalement à l'hypothèse d'une espèce regroupant les grands chevaux de type mosbachensis en y distinguant des sous espèces comme à Lunel-Viel, Caune de l'Arago², Camp-de-Peyre et La Micoque.

\section{BIBLIOGRAPHIE}

ARCELIN A. 1890 - Les nouvelles fouilles de Solutré (près de Mâcon, Saône-et-Loire). L'

Anthropologie, Paris, tome 1, p.295 - 313.

AUGUSTE P.1995 - Cadres biostratigraphiques et paléoécologiques du peuplement humain dans la France septentrionale durant le Pléistocène, apport de l'étude paléontologique des grands mammifères du gisement de Biache-Saint-Vaast (Pas-de-Calais). Doctorat de $3^{\mathrm{e}}$ cycle, Institut de Paléontologie Humaine, Paris, $404 \mathrm{p}$.

AZZAROLI A. 1982 - On Villafranchian Palaearctic Equids and their allies. Estratto dalla Palaeontographia Italica, nuova serie vol. XLII, Anni 1980-1981, p.74-97, 4 pl.

BELLAI D. 1995 - Techniques d'exploitation du Cheval à la Caune de l'Arago (Tautavel, Pyrénées Orientales). Paléo, $\mathrm{n}^{\circ}$ 7, édit. SAMRA (Les Eyzies, France), p.139-155.

BONIFAY M.-F. 1980 - Le Cheval du Pléistocène moyen des grottes de Lunel-Viel (Hérault) Equus mosbachensis palustris n.ssp. Gallia Préhistoire, 23, 2, Paris, p.233-281.

BORDES F. et PRAT F. 1965 - Observation sur les faunes du Riss et du Würm I en Dordogne. L' Anthropologie, t. 69, n 1-2, p.31-46.

BREUIL H. 1932 - Les industries à éclats du Paléolithique ancien : le Clactonien. Préhistoire, t. 1, fasc. 2, p.125-190.

BRÜNING H. 1974 - Das Quatär-Profil im Dyckerhoff-Steinbruch, Wiesbaden-Hessen. Reinh-Main. Forsch., Frankfurt/Main, ${ }^{\circ}$ 78, p.57-81.

BRÜNING H. 1978 - Zur Untergliederung der Mosbacher Terrassenabfolge und zum klimatischen Stellenwert der Mosbacher Tierwelt im Rahmen des Cromer-Komplexes. Mz. Naturw. Arch., Mainz, $\mathrm{n}^{\circ} 16, \mathrm{p} .143-190$.

CARBONELL ROURA E., ROSAS GONZALES A. et DIEZ FERNANDEZ-LOMANA J.C. (Ed.) 1999 - Atapuerca :

Occupaciones humanas y paleoecologia del yacimiento de Galeria. Aqueologia en Castilla y Leon, $\mathrm{n}^{\circ} 7$, $390 \mathrm{p}$.

CHAUVET G. et RIVIÈRE E. 1896 - Gisement quaternaire de La Micoque. C. R. Académie des Sciences, p. $1-3$.

CHAUVET G. et RIVIÈRE E. 1897 - Station quaternaire de La Micoque (Dordogne). Assoc. Franç. pour l'Avancement des Sciences, $26^{\mathrm{e}}$ Session, Saint-Étienne, p.697-708. 
CRÉGUT E. 1979 - La faune de mammifères du Pléistocène moyen de la Caune de l'Arago à Tautavel, Pyrénées Orientales. Thèse de $3^{\mathrm{e}}$ cycle, Université de Provence, Marseille, 2 tomes, 380 p., 193 tabl., 48 photos.

CRÉGUT E. 1980 - Equus mosbachensis tautavelensis nov. subsp., un nouvel Equidae (Mammalia, Perissodactyla) du gisement Pléistocène moyen anté-rissien de la Caune de l'Arago (Tautavel, Pyrénées Orientales, France). Geobios, Lyon, 13, 1, p.121-127, 2 tabl., 1 pl.

DAVID P. et PRAT F. 1962 - Sur la présence d'un Cheval de grande taille dans certains horizons moustériens de la station de la Chaise de Vouthon (Charente). Suppl. au Bull. de la Soc. Archéologique et Historique de la Charente, $\mathrm{n}^{\circ}$ 7, p.63-76, 3 pl. DEBENATH A. et RIGAUd J.-Ph. 1986 - Le gisement de La Micoque. In Rigaud J.-Ph. (dir.) Informations archéologiques en Aquitaine. Gallia Préhistoire, t. 29, fasc. 2, p.233-258.

DELPECH F. 1995 - La Micoque, Les Eyzies (Dordogne). La faune. Rapport 1995 SRA Aquitaine.

DELPECH F. 1999 - Rapport sur les travaux de biostratigraphie effectués en 1999. In J.-Ph. Rigaud, Rapport du Projet Collectif de Recherche «Litho-et Biostratigraphie de quelques sites de référence périgourdins ", document interne SRA Aquitaine, non publié.

DELPECH F., LE TENSORER J.-M., PINEDA R. et PRAT F. 1978 - Un nouveau gisement du Pléistocène moyen : Camp-de-Peyre à Sauveterre-la-Lémance (Lot-et-Garonne). C. R. Acad. Sc. Paris, t. 286, p. 1101-1103.

DELPECH F., GENESTE J.-M., RIGAUD J.-Ph. et TEXIER J.-P.1995 - Les industries antérieures à la dernière glaciation en Aquitaine septentrionale : chronologie, paléoenvironnements, technologie, typologie et économie de subsistance. In Gabori M. et al. (Ed.), Les industries à pointe foliacées d'Europe Centrale. Actes du Colloque de Miskolc, Paléo, supplément $n^{\circ} 1$, édit. SAMRA, Les Eyzies, p.133-163, 11 fig., 3 tabl.

EISENMANN V. 1980 - Les Chevaux (Equus sensus lato) fossiles et actuels : crânes et dents jugales supérieures. Cah. Paleont., CNRS, Paris, 164 p., 72 tabl., 22 pl.

EISENMANN V. 1982 - Le Cheval et ses proches parents : évolution et phylogénie. $8^{\mathrm{e}}$ journée d'étude équine, C.E.R.E.O.P.A., Paris, p.9-26.

EISENMANN V. 1991 - Les chevaux quaternaires européens (Mammalia, Perissodactyla). Taille, typologie, biostratigraphie et taxonomie. Geobios, $n^{\circ} 24$, fasc. 6, p.747-759.

EISENMANN V., CREGUT-BONNOURE E. et MOIGNE A.-M. 1985 - Equus mosbachensis et les grands Chevaux de la Caune de l'Arago et de Lunel-Viel : crâniologie comparée. Bull. Mus. Nat. Hist. Nat., Paris, $4^{\mathrm{e}}$ série, 7, section C, $\mathrm{n}^{\circ}$ 2, p.157-173.

FALGUERES C., BAHAIN J.-J., SALEKI H. 1997 - U-Series and ESR Dating of Teeth from Acheulian and Mousterian Levels at La Micoque (Dordogne, France). Journal of Archaeological Science, 24, p. 537-545.

FALGUERES C., YOKOYAMA Y., SHEN G., BISCHOFF J.L., KU T.-L. et DE LUMLEY H. 2004 - New U-series dates at the Caune de l'Arago, France. Journal of Archaeological Science, vol. 31, n 7, p.941-952.

FERNANDEZ P.2001 - Étude paléontologique et archéologique des niveaux d'occupations moustériens au Bau de l'Aubesier (Monieux, Vaucluse): implications biochronologiques et palethnologiques. Thèse de Doctorat de l’Université Claude-Bernard Lyon 1, 3 vol., 286 p., 151 fig., 9 pl., 239 tabl.

FOSSE P.1994 - Taphonomie paléolithique : les grands mammifêres de Soleilhac (Haute-Loire) et de LunelViel (Hérault). Thèse de Doctorat de l'Université de Provence-Aix-Marseille I, 257 p. 
GROMOVA V. 1949a - Histoire des chevaux (genre Equus) de l'ancien monde. Première partie : revue et description des formes. Travaux Inst. Paléontol. Acad. Sci. URSS, t. 17, n 1, 373 p., Trad. Pietresson de Saint-Aubin. Annales du Centre d'Études et de Documentation Paléontologiques, 1955, nº 13.

GROMOVA V. 1949b - Le Genre Equus. Tableaux, Figures et Planches. Annales du Centre d'Études et de Documentation Paléontologiques, Mai 1955, n 13, 17 pl., 31 tabl.

GRÜN R., MELLARS P., LAVILLE H. 1991 - ESR Chronology of a 100,000-year archaeological sequence at Pech de l'Azé II, France. Antiquity nº6, p.544-551.

GUADELLI, J.-L. 1986 - Révision de la sous espèce Equus caballus gallicus, Contribution du cheval à la connaissance des paléoenvironnements. $11^{e}$ Réunion des Sciences de la Terre, Clermont-Ferrand, Soc. Géol. Fr. édit., Paris, p.85.

GUADELLI J.-L. 1987 - Contribution à l'étude des zoocénoses préhistoriques en Aquitaine (Würm ancien et interstade würmien). Thèse de Doctorat, Université de Bordeaux I, t. I, 558 p.

GUADELLI J.-L. 1989 - Les chevaux de Solutré (Saône et Loire, France). In Datation et Caractérisation des Milieux Pléistocènes. Actes des Symposiums 11 et 17 de la $11^{\mathrm{e}}$ R.S.T., Clermont-Ferrand, 1986, Cahier du Quaternaire, $\mathrm{n}^{\circ}$ 16, CNRS, p.261-336, 9 fig., 62 tabl.

GUADELLI J.-L. et PRAT F. 1995 - Le Cheval du gisement Pléistocène moyen de Camp-de-Peyre (Sauveterre-la-Lémance, Lot-et-Garonne) : Equus mosbachensis campdepeyri nov. ssp. Paléo, édit. SAMNPRA, Les Eyzies de Tayac, $\mathrm{n}^{\circ}$ 7, p.85-121.

GUÉRIN C. 1996 - Famille des Equidae. In Guérin C. \& Patou-Mathis M. (dir.), Les grands mammifêres plio-Pléistocènes d'Europe. Édit. Masson, Paris, p.121-136.

HAUSER O. 1907 - La Micoque. Les nouvelles fouilles de La Micoque, Dordogne, et leurs résultats pour la connaissance de la civilisation paléolithique. Dédiée à la Société anthropologique de Cologne pour l'ouverture du Musée de Cologne le 28 Juillet 1907 Traduction.

LANGLOIS A. 2004 - Au sujet du Cheval de La Micoque (Dordogne) et des comportements humains de subsistance au Pléistocène moyen dans le Nord-Est de l'Aquitaine. Thèse de Doctorat, Université Bordeaux I, $383 \mathrm{p}$.

LAVILLE H. et RIGAUD J.-Ph. 1976 - Le gisement de La Micoque. In Rigaud J.-Ph. et Vandermeersch B. (sous la direction de). Livret-guide de l'Excursion A 4. Sud-Ouest (Aquitaine et Charente). IX ${ }^{e}$ congrès de l'Union Internationale des Sciences Préhistoriques et Protohistoriques, Paris, p.49-57.

LEROI-GOURHAN A. 1997 - Dictionnaire de la Préhistoire. Quadrige Presse Universitaires de France, Paris, $1222 \mathrm{p}$.

LUMLEY M.A. et H. de 1971 - Découverte de restes humains anténéandertaliens datés du Riss à la Caune de l'Arago (Pyrénées Orientales). C.R. Acad. Sc. Paris, série D, t. 272, n 9, p.1739-1742, 2 fig.

MOURER-CHAUVIRE C. 1980 - Le gisement pléistocène supérieur de la grotte de Jaurens à Nespoul (Corrèze, France) : les Équidés (Mammalia, Perissodactyla). Nouv. Arch. Mus. Hist. nat. Lyon, 18, p. 17-60.

MUSIL R. 1977 - Die Equidenreste aus den Travertinen von Taubach. Quatärpaläontologie, Berlin, 5, p.369-380.

PEYRONY D. 1938 - La Micoque. Les fouilles récentes - leur signification. Bull. de la Soc. Préhistorique Française, $\mathrm{n}^{\circ}$ 6, p.257-288.

PRAT F. 1968 - Recherches sur les Équidés pléistocènes en France. Thèse de doctorat d'État ès Sciences Naturelles, nº 226, Faculté des Sciences de Bordeaux, 4 vol., 696 p., 149 fig., 126 tabl. 
PRAT F. 1969 - Le Cheval de Solutré, Equus caballus gallicus Prat, 1968. Soc. Anthropologique du Sud-Ouest, Bordeaux, 4, 6 p., 2 fig., 3 tabl.

REICHENAU W. Von (1915) - Beiträge zur näheren Kenntnis fossiler Pferde aus deutschem Pleistozän. Insbesondere über die Entwicklung und die Abkaustadien des Gebi_vom

Hochterrassenpferd (Equus mosbachensis v. R.). Abh. hess. Geol. Landesanst. Darmstadt, 7, 1, p.1-155. RIGAUD J.-Ph. 1984 - Informations archéologiques en Aquitaine. Gallia Préhistoire, t. 27, fasc. 2, p. 269-306.

RIGAUD J.-Ph. (dir.) 1988 - La Grotte Vaufrey, paléoenvironnement, chronologie, activités humaines. Mémoires de la Société Préhistorique Française, t. 19, 616 p.

SCHWARCZ H.-P. \& GRÜN R. 1988 - ESR Dating of Level L2/3 at La Micoque (Dordogne, France): Excavations of Debénath and Rigaud. Geoarchaeology: An International Journal, 2 (4), p.293-296.

SPASSOV N. et ILLIEV N. 1997 - The wild horses of Eastern Europe and the polyphyletic origin of the domestic horse. Anthropozoologica, n 25-26, p.753-761, 2 fig.

TEXIER J.-P. et BERTRAN P. 1993 - Nouvelle interprétation paléoenvironnementale et chronostratigraphique du site paléolithique de La Micoque (Dordogne). Implications archéologiques. $C$. R. Acad. Sci. Paris, t. 316, Série II, p. 1611-1617.

\section{ANNEXES}

Tableau 2 - La Micoque. Répartition stratigraphique des restes osseux déterminés (in Delpech 1995).

Table 2 - La Micoque. Stratigraphical distribution of determined bone remains (Delpech report 1995).

\begin{tabular}{|l|c|c|c|c|c|c|c|c|c|}
\hline & $\mathbf{E}$ & $\mathbf{F}$ & $\mathbf{H}$ & $\mathbf{I}$ & $\mathbf{J}$ & $\mathbf{K}$ & $\mathbf{L}$ & Total & $\%$ \\
\hline Rangifer tarandus & $11+9^{*}$ & $1^{*}$ & $2+2^{*}$ & & $1+1^{*}$ & $1^{*}$ & $10+3^{*}$ & 41 & 3,9 \\
\hline Cervidés indéterminés & $7+1^{*}$ & & 5 & & & & 1 & 14 & 1,3 \\
\hline Cervus sp. & $4+5^{*}$ & & $1+2^{*}$ & & $1^{*}$ & & & 13 & 1,2 \\
\hline Bovidés indéterminés & 1 & & 1 & & & & & 2 & 0,2 \\
\hline Bovinae & $15+1^{*}$ & 1 & $13+2^{*}$ & 3 & $7+2^{*}$ & & 1 & 45 & 4,3 \\
\hline Rupicapra rupicapra & $1^{*}$ & & & & & & & 1 & 0,1 \\
\hline Equidés indéterminés & 12 & & 9 & 88 & 23 & & 5 & 137 & 13 \\
\hline Equus caballus & $287+17^{*}$ & $4+1^{*}$ & $97+14^{*}$ & $19+2^{*}$ & $208+22^{*}$ & $12+2^{*}$ & $107+2^{*}$ & 794 & 75,3 \\
\hline Equus hydruntinus & & & & & $1^{*}$ & & & 1 & 0,1 \\
\hline Rhinocerotidae & & & & 1 & & & & 1 & 0,1 \\
\hline Elephantidae & $1^{*}$ & & & & & & & 1 & 0,1 \\
\hline Oryctolagus cuniculus & & & 1 & & & & & 1 & 0,1 \\
\hline Canis lupus & 1 & & & & & & & 1 & 0,1 \\
\hline Ursus sp. & 1 & & & & & & 2 & 3 & 0,3 \\
\hline Total & 374 & 7 & 149 & 113 & 266 & 15 & 131 & 1055 & 100 \\
\hline
\end{tabular}

*: Nombre de pièces pour lesquelles la détermination taxonomique est vraisemblable mais non certaine.

*: Number of items, whose taxonomic determination is probable but not ascertained. 
Tableau 3 - Grotte Vaufrey. Répartition stratigraphique des restes déterminés. Table 3 - Grotte Vaufrey. Stratigraphical distribution of determined remains.

\begin{tabular}{|c|c|c|c|c|c|c|c|c|c|c|c|c|c|}
\hline & XV à XII & $\mathbf{X I}$ & $\mathrm{X}$ & IX & VIII & VII & VI & V & IV & III & II & $I$ & Total \\
\hline Sus scrofa & & 1 & 1 & & & & & & & & & & 2 \\
\hline Cervus elaphus & & 37 & 69 & 71 & 833 & 232 & 76 & 36 & 60 & 5 & 17 & 9 & 1445 \\
\hline Capreolus capreolus & & 4 & 9 & & 7 & & 1 & & 1 & & 2 & 1 & 25 \\
\hline Rangifer tarandus & & & & & & & & & 2 & 4 & 46 & 116 & 168 \\
\hline Autres cervidés & 6 & & & 1 & & 3 & & & & & & & 10 \\
\hline Saiga tatarica & & & & & & & & & 1 & & & & 1 \\
\hline Rupicapra rupicapra & & & & 4 & 9 & 7 & 1 & & & & & & 21 \\
\hline Capra sp. & & & & & & & & & 8 & 4 & 36 & 2 & 50 \\
\hline Hemitragus cf. bonali & & 4 & 11 & 8 & 129 & 21 & 4 & 4 & & & & & 181 \\
\hline Bos ou Bison & & & 4 & & 6 & 5 & 3 & 1 & 5 & 3 & & 1 & 28 \\
\hline Bison sp. & & & & & & 1 & & & & & & & 1 \\
\hline Elephantidae & & & & & 1 & 1 & 1 & 1 & & & & & 4 \\
\hline Rhinocerotidae & & 2 & 18 & & & & 2 & & 2 & & & & 24 \\
\hline Equidae & 2 & 7 & 1 & 6 & 54 & 42 & 8 & 7 & 38 & 2 & 3 & 11 & 181 \\
\hline Felidae & 2 & 1 & 4 & 7 & 27 & & & & 8 & & 5 & & 54 \\
\hline Hyaenidae & & 1 & & 1 & & & & & & & 1 & & 3 \\
\hline Canis ou Cuon & 1 & & & & 26 & & 2 & 3 & & & & & 32 \\
\hline Canis lupus subsp. & & 18 & 28 & 8 & 2 & & & & 9 & 1 & 6 & & 72 \\
\hline Vulpes vulpes & & 3 & 1 & & 25 & 1 & & & 1 & 1 & 1 & & 33 \\
\hline Vulpes ou Alopex & & 8 & 4 & 1 & 1 & 5 & 3 & & 3 & & 8 & 6 & 39 \\
\hline Autres canidés & 3 & 2 & & & 29 & & & & & & & & 34 \\
\hline Ursus deningeri & $>549$ & & & & & & & & & & & & $>549$ \\
\hline Ursus sp. & & 7 & 5 & 6 & 9 & & 1 & 4 & 2 & 2 & 1 & 1 & 38 \\
\hline Autres ursidés & & 1 & 1 & & 7 & & 2 & 2 & 1 & & 2 & & 16 \\
\hline
\end{tabular}

Les couches XV à IV datent du Pléistocène moyen (d'après Delpech 1999).

The layers XV to IV dated from middle Pleistocene (after Delpech 1999).

Tableau 4 - Pech-de-l'Azé II. Répartition des restes fauniques au sein des couches rissiennes (d'après Prat 1968).

Table 4 - Pech de l'Azé II. Distribution of faunal remains within rissien layers (after Prat 1968).

\begin{tabular}{|l|c|c|c|c|}
\cline { 2 - 5 } \multicolumn{1}{c|}{} & Couche 9 & Couche 8 & Couche 7 & Couche 6 \\
\hline Ursus sp. & $4,70 \%$ & $3,20 \%$ & $18,30 \%$ & $6,80 \%$ \\
\hline Canis lupus & $2 \%$ & $6,30 \%$ & $4,60 \%$ & $1,10 \%$ \\
\hline Vulpes vulpes & & & & $2,30 \%$ \\
\hline Hyaena spelaea & & $1 \%$ & $1,70 \%$ & $1,10 \%$ \\
\hline Sus scrofa & & $1 \%$ & & \\
\hline Capreolus & $0,70 \%$ & $4,20 \%$ & $9,10 \%$ & $2,30 \%$ \\
\hline Cervus elaphus & $22,30 \%$ & $19,40 \%$ & $13,70 \%$ & $31,90 \%$ \\
\hline Cervus sp. & $0,70 \%$ & & & \\
\hline Megaceros & & $1 \%$ & $0,60 \%$ & \\
\hline Bovidés & $16,20 \%$ & $25,10 \%$ & $21,10 \%$ & $3,50 \%$ \\
\hline Capra sp. & & & & $1,10 \%$ \\
\hline Rupicapra rupicapra & & & & $1,10 \%$ \\
\hline Equus caballus & $43,90 \%$ & $32 \%$ & $22,90 \%$ & $46,60 \%$ \\
\hline Rhinoceros mercki & $9,50 \%$ & $6,80 \%$ & $8 \%$ & $1,10 \%$ \\
\hline Nombre total de restes & 148 & 191 & 179 & 116 \\
\hline
\end{tabular}

Couches 9 à 6 = stade isotopique 6 (Grün et al. 1991).

Layers 9 to $6=$ OIS 6 (Grün et al. 1997)

Tableau 5 - Répartition stratigraphique des restes de chevaux exhumés lors des dernières fouilles (couche inconnue ou vestiges remaniés) (détermination année 2004).

Table 5 - Stratigraphical distribution of determined faunal remains of horses found during the latest excavations. (unidentified layer or rehandled remains) (date of determinations: 2004).

\begin{tabular}{|c|c|c|c|c|c|c|c|c|c|c|c|}
\hline & D & $E$ & $F$ & $\mathbf{G}$ & H & $I$ & J & $\mathrm{K}$ & $\mathrm{L}$ & * & Total \\
\hline Os & 4 & 372 & 6 & 6 & 276 & 15 & 381 & 17 & 68 & 62 & 1207 \\
\hline Dents & & 83 & 1 & & 41 & & 89 & & 18 & 7 & 239 \\
\hline Os + Dents & 4 & 455 & 7 & 6 & 317 & 15 & 470 & 17 & 86 & 69 & 1446 \\
\hline
\end{tabular}


Tableau 6 - P2 supérieures. Dimensions occlusales et indice protoconique (note 2). Table 6 - Upper P2. Occlusal measurements and protoconic coefficient (note 2).

\begin{tabular}{|c|c|c|c|c|c|}
\hline \multirow{2}{*}{ P2I } & \multirow{2}{*}{$\begin{array}{l}\text { E. mosb. } \\
\text { micoquii } \\
\text { La Micoque }\end{array}$} & \multicolumn{2}{|c|}{ Equus mosbachensis } & \multirow{2}{*}{$\begin{array}{l}\begin{array}{l}\text { E. caballus } \\
\text { germanicus }\end{array} \\
\text { Combe-Grenal } \\
\text { (Guadelli 1987) }\end{array}$} & \multirow{2}{*}{$\begin{array}{c}\begin{array}{c}\text { E. caballus } \\
\text { gallicus }\end{array} \\
\begin{array}{c}\text { Camiac } \\
\text { (Guadelli 1987) }\end{array}\end{array}$} \\
\hline & & $\begin{array}{l}\text { Biache-St-Vaast } \\
\text { (Auguste 1995) }\end{array}$ & $\begin{array}{l}\text { Bau de l'Aubesier } \\
\text { (Fernandez 2001) }\end{array}$ & & \\
\hline DMD & $\begin{array}{c}\mathrm{n}=7 \\
33,0-40,1 \\
37,38+/-1,99 \\
2,69 \\
\end{array}$ & $\begin{array}{c}\mathrm{n}=6 \\
35,2-41,3 \\
38,85+/-1,95^{\star} \\
2,44\end{array}$ & $\begin{array}{c}\mathrm{n}=13 \\
34,8-42,6 \\
39,75+/-1,12 \\
2,06 \\
\end{array}$ & $\begin{array}{c}\mathrm{n}=25 \\
36,0-41,5 \\
39,08+/-0,68 \\
1,64 \\
\end{array}$ & $\begin{array}{c}\mathrm{n}=10 \\
36,0-40,0 \\
37,84+/-1,13 \\
1,57 \\
\end{array}$ \\
\hline DVL & $\begin{array}{c}\mathrm{n}=9 \\
24,8-29,1 \\
26,49+/-0,95 \\
1,45\end{array}$ & $\begin{array}{c}\mathrm{n}=6 \\
25,3-29,2 \\
27,1+/-1,09^{*} \\
1,36\end{array}$ & $\begin{array}{c}n=15 \\
24,0-28,0 \\
25,76+/-0,66 \\
1,3\end{array}$ & $\begin{array}{c}n=33 \\
24,0-29,0 \\
26,48+1-0,37 \\
1,05\end{array}$ & $\begin{array}{c}n=10 \\
24,4-27,0 \\
25,46+/-0,75 \\
1,05\end{array}$ \\
\hline $\begin{array}{l}\text { DMD } \\
\text { protocone }\end{array}$ & $\begin{array}{c}n=8 \\
9,2-10,4 \\
9,68+/-0,32 \\
0,46\end{array}$ & $\begin{array}{c}\mathrm{n}=6 \\
8,8-10,7 \\
9,75+/-0,5^{\star} \\
0,63\end{array}$ & $\begin{array}{c}\mathrm{n}=15 \\
8,1-12,0 \\
9,53+/-0,52 \\
1,02\end{array}$ & $\begin{array}{c}n=36 \\
8,0-12,0 \\
9,75+/-0,27 \\
0,8\end{array}$ & $\begin{array}{c}n=10 \\
9,0-10,5 \\
9,76+/-0,39 \\
0,55\end{array}$ \\
\hline $\begin{array}{c}\text { Indice } \\
\text { protoconique } \\
\text { occlusal }\end{array}$ & $\begin{array}{c}n=5 \\
22,9-27,9 \\
24,65+/-1,68 \\
1,91\end{array}$ & $\begin{array}{c}n=6 \\
23,24-26,78 \\
25,15+/-1,18^{*} \\
1,48\end{array}$ & $\begin{array}{c}n=13 \\
20,57-28,17 \\
24,07+/-1,26 \\
2,32\end{array}$ & $\begin{array}{c}n=24 \\
22,5-30,6 \\
25,17+/-1,02 \\
2,42\end{array}$ & $\begin{array}{c}n=10 \\
22,5-28,38 \\
25,83+1-1,19 \\
1,66\end{array}$ \\
\hline
\end{tabular}

Tableau 7 - P3-4 supérieures. Dimensions occlusales et indice protoconique. Table 7 - Upper P3-4. Occlusal measurements and protoconic coefficient.

\begin{tabular}{|c|c|c|c|c|c|c|c|c|c|c|}
\hline \multirow[b]{2}{*}{ P3-4l } & $\begin{array}{l}\text { Emoss: } \\
\text { micoquii }\end{array}$ & $\begin{array}{c}\text { Equus } \\
\text { mosbachensis }\end{array}$ & \begin{tabular}{|c|} 
Emoshb \\
tautavelensis
\end{tabular} & $\begin{array}{c}\text { E. mosb } \\
\text { campospeyri }\end{array}$ & 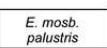 & \multicolumn{2}{|c|}{ Equus mosbachensis } & E. taubachensis & $\begin{array}{l}\text { Ecaballus } \\
\text { germanicus }\end{array}$ & $\begin{array}{c}E \text {. caballus } \\
\text { gallicius }\end{array}$ \\
\hline & La Micoque & $\begin{array}{l}\text { Mosbach } \\
\text { (Eitesnann } \\
1980)\end{array}$ & $\begin{array}{c}\begin{array}{c}\text { Caune de } \\
\text { Irago } \\
\text { Creaut 1979) }\end{array} \\
\end{array}$ & \begin{tabular}{|c|}
$\begin{array}{c}\text { Camp-di-peyre } \\
\text { (Guadele iet } \\
\text { (1995) }\end{array}$ \\
\end{tabular} & $\begin{array}{l}\text { Lunel-Viel } \\
\text { (Bonitay 1980) }\end{array}$ & \begin{tabular}{|l|}
$\begin{array}{l}\text { Biache-St-Vaast } \\
\text { (Auguste 1955) }\end{array}$ \\
\end{tabular} & \begin{tabular}{|l} 
Bau de liaubesier \\
(Fernandez 2001)
\end{tabular} & $\begin{array}{c}\text { Taubach } \\
\text { (Musil 1977) }\end{array}$ & $\begin{array}{c}\text { Combe-Grenal } \\
\text { (Guadeli i } 987 \text { ) }\end{array}$ & $\begin{array}{c}\text { Cammiac } \\
\text { (Guadelli 1987) }\end{array}$ \\
\hline DMD & $\begin{array}{c}n=15 \\
26.6 .34 .6 \\
30,34+1.1 .27 \\
2,52.27 \\
\end{array}$ & 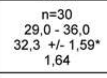 & $\begin{array}{c}n=38 \\
31,56.5 \\
34,06+1,0,08^{\circ} \\
1,21\end{array}$ & 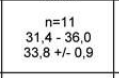 & 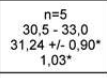 & 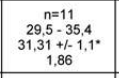 & $\begin{array}{c}n=21 \\
27,3-34.7 \\
31,42+1<0,06 \\
2,01\end{array}$ & 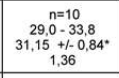 & $\begin{array}{c}n=73 \\
27.0 .335 \\
30,28+1+0,39 \\
1.7\end{array}$ & $\begin{array}{c}n=22 \\
27.0 \\
28.0 \\
28,79+1.0 .45 \\
1,02.45\end{array}$ \\
\hline DVL & $\begin{array}{c}25=15 \\
25,1-31.8 \\
28,61+1,0.93 \\
1,85\end{array}$ & $\begin{array}{c}n=29 \\
28.0 .32 .5 \\
30.5+1-0,41^{*} \\
1.12\end{array}$ & 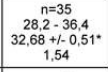 & $\begin{array}{l}n=11 \\
2,9-13,5 \\
31,6+l-1,0\end{array}$ & 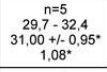 & 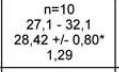 & $\begin{array}{c}n=22 \\
27,5-2.2 \\
28,96+1,0.03 \\
1,03\end{array}$ & 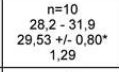 & $\begin{array}{c}n=67 \\
28,0.31,0 \\
28,33+1+0.31 \\
1,29\end{array}$ & $\begin{array}{c}n=22 \\
26,0.30 .0 \\
27,87+1,0.53 \\
1,19\end{array}$ \\
\hline MD protocon: & $\begin{array}{c}n=15 \\
11,6.15 \\
13,91,0.01 \\
1,21,61\end{array}$ & $\begin{array}{c}12=29 \\
12.0-18.5 \\
15,1+1-1,58^{*} \\
1,59\end{array}$ & 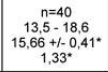 & 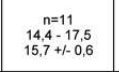 & $\begin{array}{c}n=5 \\
11,6.14,0 \\
13,16+1,0.09 \cdot \\
1,02 *\end{array}$ & \begin{tabular}{c|}
$n=9$ \\
$12,2.216,8$ \\
$14,06+1-1,01 *$ \\
1,54
\end{tabular} & $\begin{array}{c}n=22 \\
11,7.16,9 \\
14,1+1,0.061 \\
1,45\end{array}$ & $\begin{array}{c}\mathrm{n}=10 \\
13.4-17,0 \\
14,37+1,0,62 \\
1,0\end{array}$ & $\begin{array}{c}n=72 \\
11,0.180 \\
13,79+1,0.33 \\
1,43\end{array}$ & $\begin{array}{c}n=22 \\
11.5 .150 \\
13,07+150.4 \\
0,92\end{array}$ \\
\hline $\begin{array}{c}\text { Indice } \\
\text { protococoligue } \\
\text { occlusal }\end{array}$ & $\begin{array}{c}n=13 \\
37.654,1 \\
45,33+1,2,69 \\
4,95\end{array}$ & $\begin{array}{c}n=29 \\
37,68.56,06 \\
46,91+1.1 .877^{*} \\
5,13\end{array}$ & 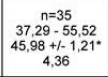 & $\begin{array}{l}n=1 \\
42,0.50 .0 \\
46,3+1-1,2\end{array}$ & 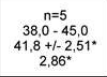 & \begin{tabular}{|c|}
$n=9$ \\
$40,67.50,15$. \\
$44,6,+1,1,82^{*}$ \\
2,78
\end{tabular} & $\begin{array}{c}n=21 \\
35.29-57,51 \\
45,4+1,2.73 \\
6,39\end{array}$ & \begin{tabular}{|c}
$n=10$ \\
$42,10.29$ \\
$46,13+5,1-1,55^{*}$ \\
2,5
\end{tabular} & $\begin{array}{c}n=71 \\
36,162,1 \\
45,74+1,1,28 \\
5,4\end{array}$ & $\begin{array}{c}n=22 \\
40,26.55,26 \\
45,48+1,1,69 \\
3,82,69\end{array}$ \\
\hline
\end{tabular}

Tableau 8 - M1-2 supérieures. Dimensions occlusales et indice protoconique. Table 8 - Upper M1-2. Occlusal measurements and protoconic coefficient.

\begin{tabular}{|c|c|c|c|c|c|c|}
\hline \multirow{2}{*}{ M1-2 I } & $\begin{array}{l}\text { E. mosb. } \\
\text { micoquii }\end{array}$ & $\begin{array}{c}\text { E. mosb. } \\
\text { tautavelensis }\end{array}$ & $\begin{array}{c}\text { E. mosb. } \\
\text { campdepeyri }\end{array}$ & $\begin{array}{l}\text { E. mosb. } \\
\text { palustris }\end{array}$ & E. taubachensis & $\begin{array}{l}\text { E. caballus } \\
\text { gallicus }\end{array}$ \\
\hline & La Micoque & $\begin{array}{l}\text { Caune de l'Arago } \\
\text { (Crégut 1979) }\end{array}$ & \begin{tabular}{|} 
Camp-de- \\
Peyre (Guadelli \\
et Prat 1995)
\end{tabular} & $\begin{array}{c}\text { Lunel-Viel } \\
\text { (Bonifay 1980) }\end{array}$ & $\begin{array}{c}\text { Taubach } \\
\text { (Musil 1977) }\end{array}$ & $\begin{array}{c}\text { Camiac } \\
\text { (Guadelli 1987) }\end{array}$ \\
\hline DMD & $\begin{array}{c}\mathrm{n}=13 \\
26,8-34,0 \\
29,33+/-1,29 \\
2,38\end{array}$ & $\begin{array}{c}\mathrm{n}=29 \\
26,0-35,0 \\
30,32+/-0,81^{*} \\
2,23\end{array}$ & $\begin{array}{c}n=15 \\
28,0-31,5 \\
29,8+/-0,6 \\
\end{array}$ & $\begin{array}{c}\mathrm{n}=9 \\
26,4-33,6 \\
28,57+/-1,45^{\star} \\
2,21^{\star}\end{array}$ & $\begin{array}{c}\mathrm{n}=15 \\
26,8-32,8 \\
29,51+/-0,85^{\star} \\
1,69\end{array}$ & $\begin{array}{c}n=33 \\
23,7-30,6 \\
25,76+/-0,45 \\
1,28\end{array}$ \\
\hline DVL & $\begin{array}{c}\mathrm{n}=10 \\
24,2-28,5 \\
26,8+/-0,96 \\
1,55\end{array}$ & $\begin{array}{c}\mathrm{n}=23 \\
28,5-33,0 \\
31,19+/-0,54^{\star} \\
1,31\end{array}$ & $\begin{array}{c}n=14 \\
26,5-31,2 \\
29,3+1-0,6\end{array}$ & $\begin{array}{c}\mathrm{n}=9 \\
28,4-30,5 \\
29,46+/-0,54^{*} \\
0,83^{*}\end{array}$ & $\begin{array}{c}\mathrm{n}=14 \\
23,4-29,7 \\
26,26+/-0,8^{*} \\
1,52\end{array}$ & $\begin{array}{c}\mathrm{n}=33 \\
24,2-28,2 \\
26,46+/-0,35 \\
0,99\end{array}$ \\
\hline $\begin{array}{l}\text { DMD } \\
\text { protocone }\end{array}$ & $\begin{array}{c}\mathrm{n}=12 \\
11,6-16,2 \\
13,79+/-0,74 \\
1,30\end{array}$ & $\begin{array}{c}\mathrm{n}=26 \\
12,5-18,0 \\
15,00+/-0,53^{\star} \\
1,38^{*}\end{array}$ & $\begin{array}{c}n=15 \\
13,0-16,7 \\
14,7+/-0,5 \\
\end{array}$ & $\begin{array}{c}n=9 \\
12,0-15,3 \\
13,63+/-0,63^{*} \\
0,96^{*}\end{array}$ & $\begin{array}{c}\mathrm{n}=14 \\
13,0-16,7 \\
14,71+/-0,49^{\star} \\
0,94\end{array}$ & $\begin{array}{c}\mathrm{n}=33 \\
12,0-16,0 \\
13,76+/-0,37 \\
1,04\end{array}$ \\
\hline IP occlusal & $\begin{array}{c}n=11 \\
42,35-50,47 \\
46,81+/-1,55 \\
2,63\end{array}$ & $\begin{array}{c}\mathrm{n}=25 \\
41,66-59,01 \\
49,07+/-1,56^{\star} \\
3,99\end{array}$ & $\begin{array}{c}n=15 \\
45,1-54,8 \\
49,5+1-1,4\end{array}$ & $\begin{array}{c}n=9 \\
40,0-54,0 \\
47,56+/-2,79^{\star} \\
4,27^{*}\end{array}$ & $\begin{array}{c}\mathrm{n}=14 \\
46,26-55,67 \\
50,14+/-1,44^{\star} \\
2,76\end{array}$ & $\begin{array}{c}\mathrm{n}=33 \\
45,42-60,76 \\
53,59+/-1,29 \\
3,63\end{array}$ \\
\hline
\end{tabular}


Tableau 9 - M3 supérieures. Dimensions occlusales et indice protoconique. Table 9 - Upper M3. Occlusal measurements and protoconic coefficient.

\begin{tabular}{|c|c|c|c|c|c|c|c|c|}
\hline \multirow[b]{2}{*}{ M3/ } & \multirow{2}{*}{$\begin{array}{l}\text { E. mosb. } \\
\text { micoquii } \\
\text { La Micoque }\end{array}$} & \multirow{2}{*}{$\begin{array}{c}E . \\
\text { mosbachensis } \\
\text { Mosbach } \\
\text { (Eisenmann } \\
1980)\end{array}$} & \multirow{2}{*}{\begin{tabular}{c|}
$\begin{array}{c}\text { E. mosb. } \\
\text { tautavelensis }\end{array}$ \\
$\begin{array}{c}\text { Caune de l'Arago } \\
\text { (Crégut 1979) }\end{array}$
\end{tabular}} & \multirow{2}{*}{\begin{tabular}{|c|}
$\begin{array}{c}\text { E. mosb. } \\
\text { campdepeyri }\end{array}$ \\
$\begin{array}{l}\text { Camp-de-Peyre } \\
\text { (Guadelli et Prat } \\
\text { 1995) }\end{array}$ \\
\end{tabular}} & \multirow{2}{*}{$\begin{array}{c}\begin{array}{c}\text { E. mosb. } \\
\text { palustris }\end{array} \\
\begin{array}{c}\text { Lunel-Viel } \\
\text { (Bonifay 1980) }\end{array}\end{array}$} & \multicolumn{2}{|c|}{ Equus mosbachensis } & \multirow{2}{*}{\begin{tabular}{|c|}
$\begin{array}{c}\text { E. caballus } \\
\text { gallicus }\end{array}$ \\
$\begin{array}{c}\text { Camiac (Guadelli } \\
1987)\end{array}$
\end{tabular}} \\
\hline & & & & & & $\begin{array}{l}\text { Biache St Vaast } \\
\text { (Auguste 1995) }\end{array}$ & $\begin{array}{c}\text { Bau de l'Aubesier } \\
\text { (Fernandez } \\
\text { 2001) }\end{array}$ & \\
\hline DMD & $\begin{array}{c}\mathrm{n}=16 \\
27,2-31,5 \\
29,54+1-0,68 \\
1,39 \\
\end{array}$ & $\begin{array}{c}\mathrm{n}=7 \\
29,5-33,0 \\
30,9+1-1,01^{*} \\
1,36\end{array}$ & $\begin{array}{c}\mathrm{n}=27 \\
31,0-37,0 \\
32,87+1-0,81^{*} \\
2,16\end{array}$ & $\begin{array}{c}n=6 \\
25,5-32,0 \\
29,1+1-2,1 \\
\end{array}$ & $\begin{array}{c}n=4 \\
30,4-32,8 \\
31,43+1,111^{*} \\
1,17^{*} \\
\end{array}$ & $\begin{array}{c}n=7 \\
26,9-36,0 \\
29,01+l-2,17^{*} \\
2,93\end{array}$ & $\begin{array}{c}\mathrm{n}=22 \\
25,6-31,5 \\
28,59+1-0,63 \\
1,51\end{array}$ & $\begin{array}{c}\mathrm{n}=14 \\
25,0-32,2 \\
27,76+l-1,05 \\
1,82\end{array}$ \\
\hline DVL & $\begin{array}{c}\mathrm{n}=18 \\
22,0-26,1 \\
24,33+1-0,46 \\
0,99\end{array}$ & $\begin{array}{c}\mathrm{n}=6 \\
22,5-25,0 \\
24,2+1-0,86^{*} \\
1,08\end{array}$ & $\begin{array}{c}\mathrm{n}=26 \\
25,0-30,0 \\
27,07+1-0,46^{*} \\
1,21\end{array}$ & $\begin{array}{c}n=6 \\
20,2-26,5 \\
23,9+1-2,1\end{array}$ & $\begin{array}{c}n=4 \\
25,9-28,4 \\
26,70+l-1,12^{*} \\
1,15^{*}\end{array}$ & $\begin{array}{c}n=7 \\
21,8-27,0 \\
23,64+l-1,25^{*} \\
1,69\end{array}$ & $\begin{array}{c}\mathrm{n}=21 \\
20,0-25,7 \\
23,58+1-0,56 \\
1,31\end{array}$ & $\begin{array}{c}n=13 \\
20,7-25,2 \\
22,65+l-0,76 \\
1,25\end{array}$ \\
\hline $\begin{array}{l}\text { DMD } \\
\text { protocone }\end{array}$ & $\begin{array}{c}n=19 \\
11,4-15,6 \\
13,73+l-0,64 \\
1,43\end{array}$ & $\begin{array}{c}n=7 \\
14,0-18,0 \\
15,7+1-0,99^{*} \\
1,34\end{array}$ & $\begin{array}{c}\mathrm{n}=26 \\
13,5-19,3 \\
15,77+1-0,95^{*} \\
2,46\end{array}$ & $\begin{array}{c}n=6 \\
14,4-16,5 \\
15,5+1-0,7\end{array}$ & $\begin{array}{c}n=4 \\
14,8-16,5 \\
15,53+1-0,84^{*} \\
0,86^{*} \\
\end{array}$ & $\begin{array}{c}n=7 \\
12,3-15,5 \\
14,26+1-0,81^{*} \\
1,1\end{array}$ & $\begin{array}{c}n=22 \\
12,1-18,0 \\
14,80+1-0,58 \\
1,39\end{array}$ & $\begin{array}{c}n=14 \\
11,7-15,4 \\
13,70+l-0,06 \\
1,05\end{array}$ \\
\hline IP occlusal & $\begin{array}{c}n=16 \\
37,5-57,14 \\
47,01+l-2,56 \\
5,23\end{array}$ & $\begin{array}{c}n=7 \\
46,77-54,54 \\
50,75+1-2,01^{*} \\
2.71\end{array}$ & $\begin{array}{c}\mathrm{n}=21 \\
45,32-58,46 \\
50,31+l-1,59^{*} \\
3,71\end{array}$ & $\begin{array}{c}n=6 \\
50,0-58,8 \\
53,3+1-3,0\end{array}$ & $\begin{array}{c}\mathrm{n}=4 \\
46,0-54,0 \\
49,00+1-3,40^{*} \\
3,46^{*}\end{array}$ & $\begin{array}{c}n=7 \\
43,06-52,98 \\
49,35+1-2,72^{*} \\
3,67\end{array}$ & $\begin{array}{c}n=22 \\
42,3-59,7 \\
51,79+l-1,86 \\
4,45\end{array}$ & $\begin{array}{c}n=14 \\
41,9-54,8 \\
49,48+1-2,45 \\
4,25 \\
\end{array}$ \\
\hline
\end{tabular}

Tableau 10 - Longueur du protocône et taille de la dent des Chevaux de La Micoque par rapport à différents Équidés pléistocènes.

Table 10 - Length of protocone and tooth size of Horses of La Micoque in comparison with various Equids from Pleistocene.

\begin{tabular}{|c|c|c|}
\hline & Longueur du protocône & Taille de la dent \\
\hline $\mathrm{P} 2 /$ & I & $\begin{array}{l}\text { - Petite taille } \\
\text { - Seul Lunel-Viel est plus petit }\end{array}$ \\
\hline P3/-4/ & $\begin{array}{l}\text { Taille normale sauf Lunel-Viel } \\
\text { s'isole : il est plus court }\end{array}$ & $\begin{array}{l}\text { - Petite taille } \\
\text { - Seul Camiac est plus petit }\end{array}$ \\
\hline M1/-2/ & Très court comme à Lunel-Viel & $\begin{array}{l}\text { Longue comme chez les Grands Chevaux mais beaucoup } \\
\text { plus étroite, comparable à Taubach }\end{array}$ \\
\hline M3/ & Très court comme à Camiac & Identique aux Grands Chevaux \\
\hline
\end{tabular}

Tableau 11 - P2 inférieures. Dimensions occlusales et indice fléxidique. Table 11 - Lower P2. Occlusal measurements and flexidic coefficient.

\begin{tabular}{|c|c|c|c|c|c|c|}
\hline & $\begin{array}{l}\text { E. mosb. } \\
\text { micoquii }\end{array}$ & E. mosbachensis & $\begin{array}{c}\text { E. mosb. } \\
\text { tautavelensis }\end{array}$ & $\begin{array}{c}\text { E. mosb. } \\
\text { campdepeyri }\end{array}$ & E. mosbachensis & $\begin{array}{l}\text { E. caballus } \\
\text { germanicus }\end{array}$ \\
\hline $\mathrm{P} / 2$ & La Micoque & $\begin{array}{c}\text { Mosbach } \\
\text { (Eisenmann } \\
\text { 1980) }\end{array}$ & $\begin{array}{l}\text { Caune de l'Arago } \\
\text { (Crégut 1979) }\end{array}$ & $\begin{array}{l}\text { Camp-de-Peyre } \\
\text { (Guadelli et Prat } \\
\text { 1995) }\end{array}$ & $\begin{array}{l}\text { Bau de l'Aubesier } \\
\text { (Fernandez 2001) }\end{array}$ & $\begin{array}{l}\text { Combe-Grenal } \\
\text { (Guadelli 1987) }\end{array}$ \\
\hline DMD & $\begin{array}{c}n=8 \\
33,8-39,1 \\
36,25+/-1,37 \\
1,97\end{array}$ & $\begin{array}{c}n=19 \\
34,0-39,0 \\
37,4+1-0,72^{*} \\
1,6\end{array}$ & $\begin{array}{c}\mathrm{n}=21 \\
33,7-40,7 \\
37,97+/-0,69^{*} \\
1,61\end{array}$ & $\begin{array}{c}n=7 \\
34,5-40,0 \\
37,4+1-1,6 \\
\end{array}$ & $\begin{array}{c}n=13 \\
29,0-38,5 \\
34,5+/-1,21 \\
2,23\end{array}$ & $\begin{array}{c}n=49 \\
29,0-38,0 \\
34,17+l-0,54 \\
1,88\end{array}$ \\
\hline DVL & $\begin{array}{c}\mathrm{n}=11 \\
14,6-17,1 \\
15,68+/-0,42 \\
0,71\end{array}$ & $\begin{array}{c}n=19 \\
15,5-17,5 \\
16,5+/-0,27^{\star} \\
0,61\end{array}$ & $\begin{array}{c}\mathrm{n}=27 \\
16,8-21,9 \\
19,29+/-0,50^{\star} \\
1,34\end{array}$ & $\begin{array}{c}n=6 \\
15,9-17,2 \\
16,4+1-0,4 \\
\end{array}$ & $\begin{array}{c}n=11 \\
14,9-17,4 \\
15,84+/-0,52 \\
0,88\end{array}$ & $\begin{array}{c}n=51 \\
13,0-18,0 \\
15,39+/-0,29 \\
1,01\end{array}$ \\
\hline $\begin{array}{l}\text { DMD } \\
\text { postfléxide }\end{array}$ & $\begin{array}{c}n=11 \\
15,0-19,3 \\
16,82+1-0,73 \\
1,24\end{array}$ & $\begin{array}{c}n=19 \\
15,0-21,5 \\
18,4+1-0,77^{*} \\
1,71\end{array}$ & & $\begin{array}{c}n=4 \\
10,5-20,0 \\
14,9\end{array}$ & $\begin{array}{c}n=12 \\
14,0-18,6 \\
17,01+/-0,79 \\
1,4\end{array}$ & $\begin{array}{c}n=49 \\
14,0-20,0 \\
16,38+1-0,37 \\
1,28\end{array}$ \\
\hline $\begin{array}{c}\text { Indice } \\
\text { fléxidique }\end{array}$ & $\begin{array}{c}n=8 \\
43,39-50,79 \\
46,33+1-1,68 \\
2,42\end{array}$ & $\begin{array}{c}n=19 \\
43,7-58,1 \\
49,2+/-1,59^{*} \\
3,53\end{array}$ & & $\begin{array}{c}n=4 \\
30,0-53,3 \\
40,65\end{array}$ & $\begin{array}{c}n=12 \\
44,67-56,06 \\
49,39+/-1,71 \\
3,03\end{array}$ & $\begin{array}{c}n=47 \\
42,2-57,1 \\
47,98+l-1,00 \\
3,4\end{array}$ \\
\hline
\end{tabular}


Tableau 12 - P3-4 inférieures. Dimensions occlusales et indice fléxidique. Table 12 - Lower P3-4. Occlusal measurements and flexidic coefficient.

\begin{tabular}{|c|c|c|c|c|c|c|c|}
\hline & $\begin{array}{l}\text { E. mosb. } \\
\text { micoquii }\end{array}$ & $\begin{array}{c}E \\
\text { mosbachensis }\end{array}$ & $\begin{array}{c}\text { E. mosb. } \\
\text { tautavelensis }\end{array}$ & $\begin{array}{c}\text { E. mosb. } \\
\text { campdepeyri }\end{array}$ & $\begin{array}{c}E \\
\text { mosbachensis }\end{array}$ & E. taubachensis & $\begin{array}{l}\text { E. caballus } \\
\text { germanicus }\end{array}$ \\
\hline$P / 3-4$ & La Micoque & $\begin{array}{l}\text { Mosbach } \\
\text { (Eisenmann } \\
1980)\end{array}$ & $\begin{array}{c}\text { Caune de l'Arago } \\
\text { (Crégut 1979) }\end{array}$ & \begin{tabular}{|c|} 
Camp-de-Peyre \\
(Guadelli et Prat \\
1995)
\end{tabular} & $\begin{array}{l}\text { Bau de l'Aubesier } \\
\text { (Fernandez 2001) }\end{array}$ & $\begin{array}{l}\text { Taubach } \\
\text { (Musil 1977) }\end{array}$ & $\begin{array}{l}\text { Combe-Grenal } \\
\text { (Guadelli 1987) }\end{array}$ \\
\hline DMD & $\begin{array}{c}n=41 \\
28,2-35,3 \\
31,70+/-0,47 \\
1,54\end{array}$ & $\begin{array}{c}\mathrm{n}=46 \\
29,5-36,0 \\
32,4+/-0,43^{*} \\
1,5\end{array}$ & $\begin{array}{c}\mathrm{n}=57 \\
28,5-39,5 \\
33,37+1-0,44 \\
1,68\end{array}$ & $\begin{array}{c}\mathrm{n}=12 \\
31,0-34,5 \\
33,4+1-0,6 \\
\end{array}$ & $\begin{array}{c}\mathrm{n}=39 \\
27,7-33,5 \\
30,69+1-0,52 \\
1,64\end{array}$ & $\begin{array}{c}n=9 \\
28,6-32,4 \\
30,87+/-0,85 \\
1,31\end{array}$ & $\begin{array}{c}n=120 \\
25,5-34,0 \\
29,97+1-0,31 \\
1,65\end{array}$ \\
\hline DVL & $\begin{array}{c}n=37 \\
14,3-20,3 \\
17,45+/-0,41 \\
1,26\end{array}$ & $\begin{array}{c}n=44 \\
16,0-20,5 \\
17,9+1-0,29^{*} \\
0,98\end{array}$ & $\begin{array}{c}n=56 \\
18,0-23,8 \\
21,48+l-0,33 \\
1,26\end{array}$ & $\begin{array}{c}n=9 \\
18,0-20,3 \\
19,4+1-0,4\end{array}$ & $\begin{array}{c}n=36 \\
14,0-20,5 \\
17,68+l-0,36 \\
1,1\end{array}$ & $\begin{array}{c}n=9 \\
16,1-19,3 \\
17,51+l-0,7 \\
1,08\end{array}$ & $\begin{array}{c}\mathrm{n}=112 \\
15,0-21,0 \\
17,54+1-0,17 \\
0,92\end{array}$ \\
\hline $\begin{array}{l}\text { DMD } \\
\text { postflexide }\end{array}$ & $\begin{array}{c}\mathrm{n}=47 \\
10,0-17,8 \\
14,90+1-0,51 \\
1,78\end{array}$ & $\begin{array}{c}\mathrm{n}=45 \\
11,5-17,5 \\
14,9+1-0,43^{*} \\
1,48\end{array}$ & & $\begin{array}{c}n=6 \\
10,0-18,0 \\
15,8+1-2,5\end{array}$ & $\begin{array}{c}n=38 \\
11,2-18,3 \\
14,86+1-0,54 \\
1,68\end{array}$ & & $\begin{array}{c}\mathrm{n}=112 \\
7,0-17,0 \\
13,75+l-0,31 \\
1,62\end{array}$ \\
\hline $\begin{array}{l}\text { Indice } \\
\text { fléxidique }\end{array}$ & $\begin{array}{c}n=40 \\
35,46-57,05 \\
47,46+1-1,53 \\
4,87\end{array}$ & $\begin{array}{c}n=45 \\
35,9-53,2 \\
46,0+l-1,02^{*} \\
3,5\end{array}$ & & $\begin{array}{c}n=6 \\
32,3-52,9 \\
47,6+1-6,6\end{array}$ & $\begin{array}{c}n=38 \\
40,0-59,42 \\
48,39+1-1,44 \\
4,53\end{array}$ & & $\begin{array}{c}n=110 \\
27,5-53,2 \\
45,77+1-0,74 \\
3,9\end{array}$ \\
\hline
\end{tabular}

Tabeau 13 - M1-2 inférieures. Dimensions occlusales et indice fléxidique. Table 13 - Lower M1-2. Occlusal measurements and flexidic coefficient.

\begin{tabular}{|c|c|c|c|c|c|c|c|c|}
\hline \multirow[b]{2}{*}{ M/1-2 } & \multirow{2}{*}{$\begin{array}{l}\text { E. mosb. } \\
\text { micoquii } \\
\text { La Micoque }\end{array}$} & \multirow{2}{*}{$\begin{array}{c}E . \\
\text { mosbachensis } \\
\text { Mosbach } \\
\text { (Eisenmann } \\
1980)\end{array}$} & \multirow{2}{*}{$\begin{array}{c}\text { E. mosb. } \\
\text { tautavelensis } \\
\begin{array}{c}\text { Caune de l'Arago } \\
\text { (Crégut 1979) }\end{array}\end{array}$} & \multirow{2}{*}{\begin{tabular}{|c|}
$\begin{array}{c}\text { E. mosb. } \\
\text { campdepeyri }\end{array}$ \\
$\begin{array}{c}\text { Camp-de-Peyre } \\
\text { (Guadelli et Prat } \\
\text { 1995) }\end{array}$
\end{tabular}} & \multirow{2}{*}{$\begin{array}{c}\begin{array}{c}\text { E. mosb. } \\
\text { palustris }\end{array} \\
\begin{array}{c}\text { Lunel-Viel } \\
\text { (Bonifay 1980) }\end{array}\end{array}$} & \multicolumn{2}{|c|}{ E. mosbachensis } & \multirow{2}{*}{$\begin{array}{l}\begin{array}{c}\text { E. caballus } \\
\text { germanicus }\end{array} \\
\begin{array}{l}\text { Combe-Grenal } \\
\text { (Guadelli 1987) }\end{array}\end{array}$} \\
\hline & & & & & & $\begin{array}{l}\text { Biache-St-Vaast } \\
\text { (Auguste 1995) }\end{array}$ & \begin{tabular}{|c} 
Bau de l'Aubesier \\
(Fernandez \\
2001)
\end{tabular} & \\
\hline DMD & $\begin{array}{c}n=44 \\
26,1-34,6 \\
30,11+l-0,62 \\
2.11\end{array}$ & $\begin{array}{c}n=44 \\
27-35 \\
29,8+1-0,49^{*} \\
1.67\end{array}$ & $\begin{array}{c}\mathrm{n}=50 \\
26,7-37,7 \\
31,94+1-0,68^{*} \\
2.47\end{array}$ & $\begin{array}{c}n=13 \\
28,7-31,7 \\
30,5+1-0,4\end{array}$ & $\begin{array}{c}n=5 \\
27,0-29,3 \\
28,18+1-0,75^{*} \\
0,85^{*}\end{array}$ & $\begin{array}{c}n=2 \\
26,2-28,9 \\
27,55\end{array}$ & $\begin{array}{c}n=48 \\
25,7-32,8 \\
29,09+1-0,55 \\
1,96\end{array}$ & $\begin{array}{c}n=124 \\
21,5-33,0 \\
27,78+l-0,32 \\
1,83\end{array}$ \\
\hline DVL & $\begin{array}{c}n=43 \\
12,1-17,1 \\
15,03+1-0,39 \\
1,30\end{array}$ & $\begin{array}{c}n=42 \\
14-18 \\
16,0+1-0,29^{*} \\
0.95\end{array}$ & $\begin{array}{c}n=50 \\
17,5-23,0 \\
20,09+1-0,28^{*} \\
1,02\end{array}$ & $\begin{array}{c}\mathrm{n}=10 \\
17,0-18,5 \\
17,8+1-0,3\end{array}$ & $\begin{array}{c}n=5 \\
19,5-20,5 \\
28,18+1-0,31^{*} \\
0.36^{*}\end{array}$ & $\begin{array}{c}n=2 \\
14,2-15,5 \\
14,85+l-0,90^{*} \\
0.65\end{array}$ & $\begin{array}{c}n=45 \\
13,0-16,3 \\
15,73+l-0,57 \\
1,95\end{array}$ & $\begin{array}{c}n=122 \\
13,0-18,0 \\
15,67+l-0,17 \\
0,98\end{array}$ \\
\hline $\begin{array}{c}\text { DMD } \\
\text { postflexide }\end{array}$ & $\begin{array}{c}n=48 \\
8,4-15,5 \\
11,92+l-0,50 \\
1,76\end{array}$ & $\begin{array}{c}n=43 \\
7,5-15,0 \\
11,1+1-0,47^{*} \\
1,57\end{array}$ & & $\begin{array}{c}\mathrm{n}=7 \\
11,4-12,8 \\
11,9+1-0,4\end{array}$ & & $\begin{array}{c}n=2 \\
8,9-10,3 \\
9,6 \\
0,7\end{array}$ & $\begin{array}{c}n=44 \\
8,6-16,3 \\
11,74+1-0,58 \\
1,96\end{array}$ & $\begin{array}{c}n=121 \\
7,0-14,1 \\
10,45+1-0,25 \\
1,42\end{array}$ \\
\hline $\begin{array}{l}\text { Indice } \\
\text { fléxidique }\end{array}$ & $\begin{array}{c}n=40 \\
31,45-49,0 \\
39,86+l-1,33 \\
4,30\end{array}$ & $\begin{array}{c}n=44 \\
22,2-45,6 \\
36,9+1-1,45^{*} \\
4,92\end{array}$ & & $\begin{array}{c}n=7 \\
37,5-42,7 \\
39,9+1-1,4\end{array}$ & & $\begin{array}{c}n=2 \\
33,97-35,64 \\
34,8\end{array}$ & $\begin{array}{c}n=40 \\
31,07-48,67 \\
39,88+1-1,48 \\
4,8\end{array}$ & $\begin{array}{c}n=121 \\
28,6-44,4 \\
37,27+1-0,67 \\
3,73\end{array}$ \\
\hline
\end{tabular}

Tableau 14 - M3 inférieures. Dimensions occlusales et indice fléxidique. Table 14 - Lower M3. Occlusal measurements and flexidic coefficient.

\begin{tabular}{|c|c|c|c|c|c|c|c|}
\hline \multirow[b]{2}{*}{$M / 3$} & \multirow{2}{*}{$\begin{array}{c}\begin{array}{c}\text { E. mosb. } \\
\text { micoquii }\end{array} \\
\text { La Micoque }\end{array}$} & \multirow{2}{*}{$\begin{array}{c}\text { E. mosbachensis } \\
\begin{array}{c}\text { Mosbach } \\
\text { (Eisenmann 1980) }\end{array} \\
\end{array}$} & \multirow{2}{*}{\begin{tabular}{|c|}
$\begin{array}{c}\text { E. mosb. } \\
\text { tautavelensis }\end{array}$ \\
$\begin{array}{c}\text { Caune de l'Arago } \\
\text { (Crégut 1979) }\end{array}$
\end{tabular}} & \multirow{2}{*}{\begin{tabular}{|c|} 
E. \\
mosbachensis \\
Bau de l'Aubesier \\
(Fernandez \\
2001)
\end{tabular}} & \multirow{2}{*}{$\begin{array}{l}\text { E. caballus } \\
\text { germanicus } \\
\\
\text { Combe-Grenal } \\
\text { (Guadelli 1987) }\end{array}$} & \multicolumn{2}{|c|}{ E. caballus gallicus } \\
\hline & & & & & & $\begin{array}{c}\text { Camiac } \\
\text { (Guadelli 1987) }\end{array}$ & $\begin{array}{l}\text { Jaurens (Mourer- } \\
\text { Chauviré 1980) }\end{array}$ \\
\hline DMD & $\begin{array}{c}\mathrm{n}=13 \\
30,7-35,3 \\
33,53+l-0,68 \\
1,26\end{array}$ & $\begin{array}{c}\mathrm{n}=17 \\
33,0-41,5 \\
36,3+1-0,9^{\star} \\
1,9\end{array}$ & $\begin{array}{c}\mathrm{n}=32 \\
34,4-40,5 \\
37,28+1-0,59^{\star} \\
1,71\end{array}$ & $\begin{array}{c}\mathrm{n}=16 \\
31,5-36,6 \\
33,68+1-0,72 \\
1,46\end{array}$ & $\begin{array}{c}\mathrm{n}=48 \\
30,0-38,0 \\
33,48+1-0,52 \\
1,77\end{array}$ & $\begin{array}{c}\mathrm{n}=7 \\
29,3-33,3 \\
30,74+/-1,21 \\
1,31\end{array}$ & $\begin{array}{c}\mathrm{n}=29 \\
28,6-35,3 \\
32,00+1-0,71^{*} \\
1,95^{*} \\
\end{array}$ \\
\hline DVL & $\begin{array}{c}\mathrm{n}=24 \\
10,9-15,2 \\
13,58+1-0,47 \\
1,17 \\
\end{array}$ & $\begin{array}{c}n=17 \\
13,0-16,5 \\
14,9+1-0,46^{*} \\
0,96\end{array}$ & $\begin{array}{c}n=35 \\
14,5-19,3 \\
16,76+1-0,38^{\star} \\
1,15 \\
\end{array}$ & $\begin{array}{c}\mathrm{n}=15 \\
11,6-15,4 \\
14,12+1-0,59 \\
1,16 \\
\end{array}$ & $\begin{array}{c}n=48 \\
12,1-16,0 \\
13,93+/-0,26 \\
0,9\end{array}$ & $\begin{array}{c}n=8 \\
12,1-14,0 \\
13,05+/-0,57 \\
0,68\end{array}$ & $\begin{array}{c}\text { au point } P \\
n=28 \\
12,2-14,7 \\
13,17+1-0,29^{*} \\
0,79^{*}\end{array}$ \\
\hline $\begin{array}{c}\text { DMD } \\
\text { postflexide }\end{array}$ & $\begin{array}{c}n=24 \\
10,3-13,9 \\
12,10+l-0,43 \\
1,07 \\
\end{array}$ & & & & & & \\
\hline $\begin{array}{c}\text { Indice } \\
\text { fléxidique }\end{array}$ & $\begin{array}{c}n=13 \\
31,66-39,16 \\
36,14+1-1,45 \\
2.66\end{array}$ & & & & & & \\
\hline
\end{tabular}


Tabeau 15 - Scapula. Diamètres antéro-postérieur et transversal de la cavité glénoïde des différents chevaux de type mosbachensis et caballus.

Table 15 - Scapula. Antero-posterior and transversal diameter of glenoidal cavity of different horses of mosbachensis and caballus types.

\begin{tabular}{|c|c|c|c|c|c|c|}
\hline \multirow[b]{2}{*}{ Scapula } & \multirow{2}{*}{$\begin{array}{l}\text { E. mosb. } \\
\text { micoquii } \\
\text { La Micoque }\end{array}$} & \multirow{2}{*}{$\begin{array}{c}\text { E. mosb. } \\
\text { tautavelensis } \\
\text { Caune de } \\
\text { l'Arago } \\
\text { (Crégut 1979) }\end{array}$} & \multirow{2}{*}{$\begin{array}{c}\begin{array}{c}\text { E. mosb. } \\
\text { palustris }\end{array} \\
\begin{array}{c}\text { Lunel-Viel } \\
\text { (Bonifay 1980) }\end{array}\end{array}$} & \multicolumn{2}{|c|}{ E. caballus gallicus } & \multirow{2}{*}{$\begin{array}{c}\text { E. } \\
\text { przewalskii } \\
\text { (Gromova } \\
\text { 1949b) }\end{array}$} \\
\hline & & & & $\begin{array}{c}\text { Solutré } \\
\text { (Guadelli } \\
\text { 1987) }\end{array}$ & \begin{tabular}{|c|} 
St Germain-la \\
Rivière \\
(Prat 1968)
\end{tabular} & \\
\hline $\begin{array}{l}\text { DAP cavité } \\
\text { glénoïde }\end{array}$ & $\begin{array}{c}\mathrm{n}=5 \\
57,4-62,0 \\
59,92+/-2,03 \\
2,31 \\
\end{array}$ & $\begin{array}{c}\mathrm{n}=6 \\
57,0-68,0 \\
63,2+/-3,02^{*} \\
3,78^{*} \\
\end{array}$ & $\begin{array}{c}59,5-63,0 \\
60,67+/-2,29^{*} \\
2,01^{*}\end{array}$ & 50,8 & $\begin{array}{c}n=6 \\
3,0-59,0 \\
-\end{array}$ & $\begin{array}{c}n=8 \\
50,0-58,0 \\
54,2\end{array}$ \\
\hline $\begin{array}{l}\text { DT cavité } \\
\text { glénoïde }\end{array}$ & $\begin{array}{c}n=5 \\
44,3-55,2 \\
49,74+/-3,94 \\
4,49\end{array}$ & $\begin{array}{c}\mathrm{n}=7 \\
51,0-57,5 \\
54,81+/-1,79^{*} \\
2,42^{\star}\end{array}$ & $\begin{array}{c}n=3 \\
50,5-53,0 \\
51,5+1-1,32^{*} \\
1,50^{*}\end{array}$ & 50,8 & $\begin{array}{c}n=6 \\
46,0-52,5 \\
-\end{array}$ & $\begin{array}{c}n=8 \\
43,0-49,0 \\
-\end{array}$ \\
\hline
\end{tabular}

Tableau 16 - Humérus. Diamètre antéro-postérieur minimum de la trochlée et diamètre transversal articulaire distal chez les " grands » chevaux.

Table 16 - Humerus. Antero-posterior minimal diameter of trochlea and distal articular transversal diameter among the "large" horses.

\begin{tabular}{|c|c|c|c|}
\hline \multirow{2}{*}{ Humérus } & $\begin{array}{l}\text { E. mosb. } \\
\text { micoquii }\end{array}$ & $\begin{array}{c}\text { E. mosb. } \\
\text { tautavelensis }\end{array}$ & $\begin{array}{l}\text { E. caballus } \\
\text { piveteaui }\end{array}$ \\
\hline & La Micoque & $\begin{array}{l}\text { Caune de l'Arago } \\
\text { (Crégut 1979) }\end{array}$ & $\begin{array}{l}\text { Abri Suard } \\
\text { (Prat 1968) }\end{array}$ \\
\hline $\begin{array}{c}\text { DT articulaire } \\
\text { dist. }\end{array}$ & $\begin{array}{c}n=6 \\
80,8-91,8 \\
86,98+/-3,65 \\
4,57\end{array}$ & $\begin{array}{c}\mathrm{n}=4 \\
86,0-91,0 \\
89,18+/-2,15^{*} \\
2,19\end{array}$ & $\begin{array}{c}n=6 \\
85,5-93,0 \\
88,83+/-2,51 \text { * } \\
3,14\end{array}$ \\
\hline $\begin{array}{l}\text { DAP mini } \\
\text { trochlée }\end{array}$ & $\begin{array}{c}n=6 \\
38,7-44,4 \\
42,27+/-1,69 \\
2,12\end{array}$ & & \\
\hline
\end{tabular}


Tableau 17 - Dimensions des extrémités des radio-ulnas du Cheval de La Micoque, d'Equus mosbachensis et d'Equus caballus germanicus.

Table 17 - Measurements of the extremities of the radio-ulna of the Horse of La Micoque, Equus mosbachensis and Equus caballus germanicus.

\begin{tabular}{|c|c|c|c|c|c|}
\hline \multirow{2}{*}{ Radio-ulna } & \multirow{2}{*}{$\begin{array}{l}\text { E. mosb. } \\
\text { micoquii } \\
\text { La Micoque }\end{array}$} & \multicolumn{2}{|c|}{ E. mosbachensis } & \multicolumn{2}{|c|}{ E. caballus germanicus } \\
\hline & & $\begin{array}{l}\text { Bau de l'Aubesier } \\
\text { (Fernandez 2001) }\end{array}$ & $\begin{array}{l}\text { Biache-St-Vaast } \\
\text { (Auguste 1995) }\end{array}$ & $\begin{array}{c}\text { Pair-non-Pair } \\
\text { (Prat 1968) }\end{array}$ & $\begin{array}{l}\text { Combe-Grenal } \\
\text { (Guadelli 1987) }\end{array}$ \\
\hline DAP maxi prox. & 49,$0 ; 53,1$ & & & & \\
\hline DT maxi prox. & 88,5 & & & & \\
\hline $\begin{array}{l}\text { DAP prox. } \\
\text { articulaire }\end{array}$ & $\begin{array}{c}\mathrm{n}=4 \\
40,6-46,4 \\
43,35+/-2,36 \\
2,41\end{array}$ & & 45,$5 ; 49,6$ & & \\
\hline $\begin{array}{l}\text { DT prox. } \\
\text { articulaire }\end{array}$ & 85,3 & & & & \\
\hline DAP maxi dist. & 46,5 & & & $\begin{array}{c}n=3 \\
42,0-48,5 \\
45,1\end{array}$ & 47,5 \\
\hline DT maxi dist. & 77,6 & 88,1 & 88,$2 ; 94,8$ & $\begin{array}{c}\mathrm{n}=9 \\
72,0-84,0 \\
77,8\end{array}$ & 83,5 \\
\hline $\begin{array}{l}\text { DAP dist. } \\
\text { articulaire }\end{array}$ & $\begin{array}{c}n=3 \\
30,7-38,6 \\
35,93+/-5,13 \\
4,53\end{array}$ & 44,5 & 38,$5 ; 46,5$ & $\begin{array}{c}n=8 \\
36,0-42,5 \\
39,3\end{array}$ & 37,0 \\
\hline DT dist. articulaire & 71,$7 ; 65,4$ & 74 & 73,$0 ; 82,3$ & $\begin{array}{c}\mathrm{n}=8 \\
64,0-71,0 \\
67,25\end{array}$ & 69,0 \\
\hline $\begin{array}{l}\text { DT condyle dist. } \\
\text { Radius }\end{array}$ & $\begin{array}{c}\mathrm{n}=4 \\
30,3-34,1 \\
31,85+/-1,58 \\
1,61\end{array}$ & 31,3 & 31,$0 ; 36,5$ & & \\
\hline $\begin{array}{c}\text { DT condyle dist. } \\
\text { Ulna }\end{array}$ & $\begin{array}{c}\mathrm{n}=8 \\
15,4-20,2 \\
17,93+/-1,19 \\
1,72\end{array}$ & 18,2 & 19,$1 ; 20,1$ & & \\
\hline
\end{tabular}

Tableau 18 - Dimensions des Scaphoïde et Trapézoïde. Table 18 - Measurements of Scaphoide and Trapezoide.

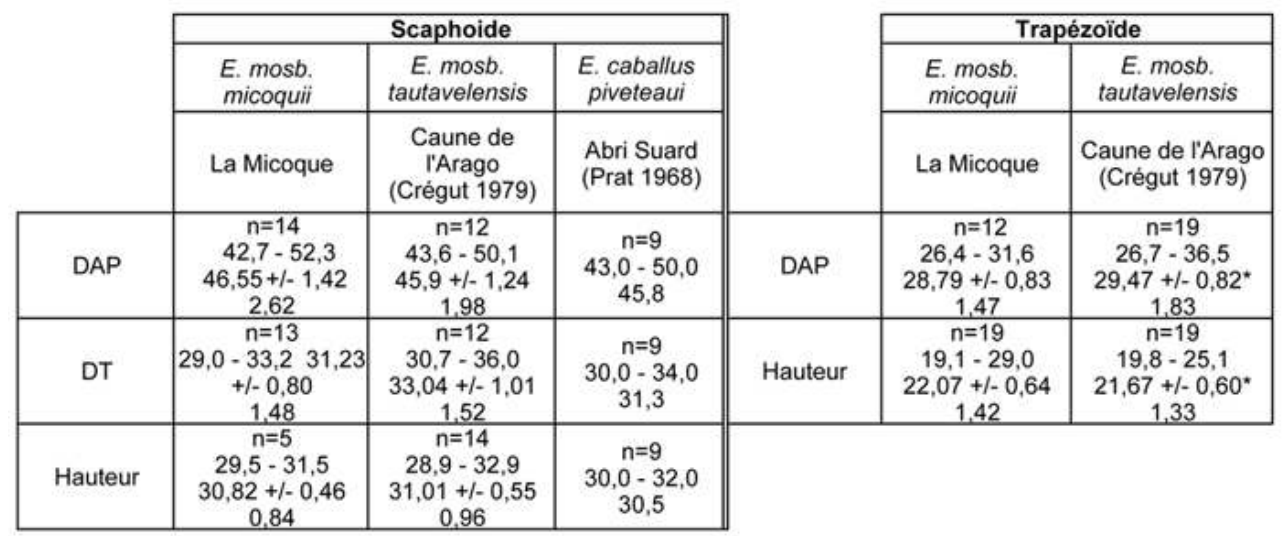


Tableau 19 - Dimensions des Semi-lunaire et Capitatum. Table 19 - Measurements of Semi-lunar and Capitatum.

\begin{tabular}{|c|c|c|c|c|c|c|c|}
\hline & \multicolumn{3}{|c|}{ Semi-lunaire } & & \multicolumn{3}{|c|}{ Capitatum } \\
\hline & $\begin{array}{l}\text { E. mosb. } \\
\text { micoquii }\end{array}$ & $\begin{array}{c}\text { E. mosb. } \\
\text { tautavelensis }\end{array}$ & $\begin{array}{l}\text { E. caballus } \\
\text { piveteaui }\end{array}$ & & $\begin{array}{l}\text { E. mosb. } \\
\text { micoquii }\end{array}$ & $\begin{array}{c}\text { E. mosb. } \\
\text { tautavelensis }\end{array}$ & $\begin{array}{l}\text { E. caballus } \\
\text { piveteaui }\end{array}$ \\
\hline & La Micoque & $\begin{array}{c}\text { Caune de l'Arago } \\
\text { (Crégut 1979) }\end{array}$ & $\begin{array}{l}\text { Abri Suard } \\
\text { (Prat 1968) }\end{array}$ & & La Micoque & $\begin{array}{c}\text { Caune de l'Arago } \\
\text { (Crégut 1979) }\end{array}$ & $\begin{array}{l}\text { Abri Suard } \\
\text { (Prat 1968) }\end{array}$ \\
\hline DAP & $\begin{array}{c}n=18 \\
40,7-44,9 \\
42,62+/-0,62 \\
1,34\end{array}$ & $\begin{array}{c}n=14 \\
38,8-47,5 \\
42,43\end{array}$ & $\begin{array}{c}n=7 \\
41,0-48,0 \\
43,3\end{array}$ & DAP & $\begin{array}{c}n=15 \\
43,3-50,5 \\
45,68+/-1,08 \\
2,13\end{array}$ & $\begin{array}{c}n=16 \\
44,0-52,8 \\
46,2+/-0,73^{*} \\
1,5\end{array}$ & $\begin{array}{c}n=10 \\
44,0-49,0 \\
46,95\end{array}$ \\
\hline DT prox. & $\begin{array}{c}\mathrm{n}=24 \\
27,7-36,3 \\
31,52+/-0,83 \\
2,07 \\
\end{array}$ & $\begin{array}{c}n=13 \\
31,7-37,7 \\
35,07 \\
\end{array}$ & $\begin{array}{c}n=7 \\
31,5-35,5\end{array}$ & DT & $\begin{array}{c}\mathrm{n}=17 \\
44,3-52,7 \\
48,58+/-1,13 \\
2,39 \\
\end{array}$ & $\begin{array}{c}\mathrm{n}=14 \\
48,0-53,8 \\
50,99+/-0,85^{\star} \\
1,62\end{array}$ & $\begin{array}{c}n=10 \\
49,0-53,0 \\
50,8\end{array}$ \\
\hline DT dist. & $\begin{array}{c}\mathrm{n}=22 \\
23,2-26,1 \\
25,0+/-0,37 \\
0,88\end{array}$ & $\begin{array}{c}n=15 \\
23,7-29,5 \\
25,57\end{array}$ & $\begin{array}{c}n=7 \\
24,5-28,0\end{array}$ & & & & \\
\hline Hauteur & $\begin{array}{c}\mathrm{n}=14 \\
27,8-31,5 \\
29,7+/-0,66 \\
1,26 \\
\end{array}$ & $\begin{array}{c}n=12 \\
29,4-32,6 \\
31,13\end{array}$ & $\begin{array}{c}n=7 \\
30,0-34,5\end{array}$ & & & & \\
\hline
\end{tabular}

Tableau 20 - Dimensions du Pyramidal, Unciforme et Pisiforme. Table 20 - Measurements of Pyramidal, Unciforme and Pisiforme.

\begin{tabular}{|c|c|c|c|c|c|c|c|c|c|}
\hline & \multicolumn{2}{|c|}{ Pyramidal } & & \multicolumn{3}{|c|}{ Unciforme } & & \multicolumn{2}{|c|}{ Pisiforme } \\
\hline & $\begin{array}{l}\text { E. mosb. } \\
\text { micoquii }\end{array}$ & $\begin{array}{l}\text { E. caballus } \\
\text { piveteaui }\end{array}$ & & $\begin{array}{l}\text { E. mosb. } \\
\text { micoquii }\end{array}$ & $\begin{array}{c}\text { E. mosb. } \\
\text { tautavelensis }\end{array}$ & $\begin{array}{l}\text { E. caballus } \\
\text { piveteaui }\end{array}$ & & $\begin{array}{l}\text { E. mosb. } \\
\text { micoquii }\end{array}$ & $\begin{array}{c}\text { E. mosb. } \\
\text { tautavelensis }\end{array}$ \\
\hline & La Micoque & $\begin{array}{l}\text { Abri Suard } \\
\text { (Prat 1968) }\end{array}$ & & La Micoque & $\begin{array}{c}\text { Caune de } \\
\text { l'Arago } \\
\text { (Crégut 1979) }\end{array}$ & $\begin{array}{l}\text { Abri Suard } \\
\text { (Prat 1968) }\end{array}$ & & La Micoque & $\begin{array}{c}\text { Caune de } \\
\text { l'Arago } \\
\text { (Crégut 1979) }\end{array}$ \\
\hline DAP & $\begin{array}{c}\mathrm{n}=16 \\
28,4-35,5 \\
31,95+1-1,15 \\
2,35\end{array}$ & $\begin{array}{c}n=5 \\
33,5-40,0 \\
35,5\end{array}$ & DAP & $\begin{array}{c}n=27 \\
26,3-36,3 \\
32,71+/-0,75 \\
1,98\end{array}$ & $\begin{array}{c}\mathrm{n}=17 \\
32,6-36,4 \\
34,66+l-1,10 \\
1,46\end{array}$ & $\begin{array}{c}\mathrm{n}=5 \\
32,0-36,5 \\
34,3+1-1,47^{\star} \\
1,68 \\
\end{array}$ & DAP & $\begin{array}{c}n=5 \\
44,8-49,4 \\
47,2+/-1,7 \\
1,98\end{array}$ & $\begin{array}{c}n=7 \\
46,4-54,5 \\
49,1+1-2,6 \\
2,87\end{array}$ \\
\hline Hauteur & $\begin{array}{c}\mathrm{n}=16 \\
24,3-30,5 \\
27,77++-0,90 \\
1,84\end{array}$ & $\begin{array}{c}n=5 \\
27,0-31,5 \\
28,9\end{array}$ & DT & $\begin{array}{c}\mathrm{n}=26 \\
18,5-27,8 \\
22,83+1-0,92 \\
2,40\end{array}$ & $\begin{array}{c}\mathrm{n}=19 \\
21,5-27,5 \\
24,69+/-1,13 \\
1,70\end{array}$ & $\begin{array}{c}\mathrm{n}=5 \\
22,0-24,5 \\
23,6+1-0,89^{\star} \\
1,02\end{array}$ & $\begin{array}{l}\text { Hauteur } \\
\text { totale }\end{array}$ & $\begin{array}{c}n=6 \\
34,5-41,8 \\
38,1+/-2,1 \\
2,61\end{array}$ & $\begin{array}{c}n=7 \\
36,3-43,8 \\
39,4+1-2,4 \\
2,69\end{array}$ \\
\hline & & & Hauteur & $\begin{array}{c}n=30 \\
21,4-25,4 \\
23,11+/-0,40 \\
1,12\end{array}$ & $\begin{array}{c}\mathrm{n}=19 \\
22,5-26,7 \\
24,44+l-0,85 \\
1,28\end{array}$ & $\begin{array}{c}\mathrm{n}=5 \\
23,5-24,5 \\
24,3+1-0,50^{\star} \\
0,57\end{array}$ & $\begin{array}{c}\text { Hauteur } \\
\text { antérieure }\end{array}$ & $\begin{array}{c}\mathrm{n}=6 \\
31,8-36,3 \\
33,3+/-1,5 \\
1,84\end{array}$ & $\begin{array}{c}\mathrm{n}=8 \\
33,0-38,5 \\
35,0+/-1,5 \\
1,90\end{array}$ \\
\hline
\end{tabular}

Tableau 21 - Dimensions des extrémités proximales des métacarpiens accessoires du Cheval de La Micoque et de quelques Équidés du Pléistocène.

Table 21 - Measurements of proximal extremities of secondary metacarpials of the Horse of La Micoque and of some Equids from Pleistocene.

\begin{tabular}{|c|c|c|c|c|c|c|c|c|c|}
\hline & \multicolumn{4}{|c|}{ Métacarpien II } & \multicolumn{5}{|c|}{ Métacarpien IV } \\
\hline & \multirow{2}{*}{$\begin{array}{l}\text { E. mosb. } \\
\text { micoquii } \\
\text { La Micoque }\end{array}$} & \multicolumn{2}{|c|}{ E. mosbachensis } & \multirow{2}{*}{$\begin{array}{c}\begin{array}{c}\text { E. mosb. } \\
\text { palustris }\end{array} \\
\begin{array}{c}\text { Lunel-Viel } \\
\text { (Bonifay 1980) }\end{array}\end{array}$} & \multirow{2}{*}{$\begin{array}{c}\begin{array}{c}\text { E. mosb. } \\
\text { micoquii }\end{array} \\
\text { La Micoque }\end{array}$} & \multirow{2}{*}{$\begin{array}{c}\begin{array}{c}\text { E. mosb. } \\
\text { tautavelensis }\end{array} \\
\text { Caune de l'Arago } \\
\text { (Crégut 1979) }\end{array}$} & \multirow{2}{*}{\begin{tabular}{|c|}
$\begin{array}{c}\text { E. mosb. } \\
\text { campdepeyri }\end{array}$ \\
$\begin{array}{c}\text { Camp-de- } \\
\text { Peyre } \\
\text { (Guadelli et } \\
\text { Prat 1995) }\end{array}$ \\
\end{tabular}} & \multicolumn{2}{|c|}{ E. caballus gallicus } \\
\hline & & $\begin{array}{c}\text { Bau de } \\
\text { l'Aubesier } \\
\text { (Fernandez } \\
\text { 2001) }\end{array}$ & $\begin{array}{l}\text { Biache-St-Vaast } \\
\text { (Auguste 1995) }\end{array}$ & & & & & $\begin{array}{c}\text { Camiac } \\
\text { (Guadelli } \\
\text { 1987) }\end{array}$ & $\begin{array}{c}\text { Solutré } \\
\text { (Guadelli 1987) }\end{array}$ \\
\hline $\begin{array}{l}\text { DAP } \\
\text { maxi }\end{array}$ & $\begin{array}{c}\mathrm{n}=21 \\
21,0-25,3 \\
23,09+1-0,59 \\
1,39\end{array}$ & 20,$5 ; 22,5$ & $\begin{array}{c}\mathrm{n}=3 \\
18,7-21,0 \\
19,9+l-1,06^{*} \\
0,94\end{array}$ & $\begin{array}{c}n=11 \\
19,5-23,5 \\
21,6+1-0,67^{*} \\
1,14\end{array}$ & $\begin{array}{c}\mathrm{n}=22 \\
18,1-25,3 \\
21,80+1-0,77 \\
1,84\end{array}$ & $\begin{array}{c}\mathrm{n}=13 \\
21,7-25,2 \\
23,61+l-0,61 * \\
1,12\end{array}$ & 23 & $22,8: 23,1$ & $\begin{array}{c}\mathrm{n}=7 \\
20,5-23,3 \\
21,74+1-0,92 \\
0,99\end{array}$ \\
\hline $\begin{array}{l}\text { mT } \\
\text { maxi }\end{array}$ & $\begin{array}{c}\mathrm{n}=22 \\
12,3-16,4 \\
14,41++1-0,46 \\
1,09\end{array}$ & 14,$3 ; 14,7$ & $\begin{array}{c}n=3 \\
13,5-16,5 \\
15,03+1,1,39^{*} \\
1,23\end{array}$ & & $\begin{array}{c}\mathrm{n}=22 \\
13,1-19,0 \\
15,70+1-0,71 \\
1,69\end{array}$ & $\begin{array}{c}n=13 \\
15,8-20,5 \\
18,18+l-0,67^{*} \\
1,24\end{array}$ & 18,1 & & $\begin{array}{c}\mathrm{n}=7 \\
14,4-16,5 \\
15,70+1-0,75 \\
0,81\end{array}$ \\
\hline
\end{tabular}


Tableau 22 - Dimensions des extrémités des métacarpiens III du Cheval de La Micoque, d'Equus mosbachensis et d'Equus caballus.

Table 22 - Measurements of metacarpals III extremities of the Horse of La Micoque, of Equus mosbachensis and Equus caballus.

\begin{tabular}{|c|c|c|c|c|c|c|c|c|c|c|}
\hline \multirow[b]{2}{*}{ Métacarpien III } & \multirow{2}{*}{$\begin{array}{c}\begin{array}{c}\text { E. mosb. } \\
\text { micoquii }\end{array} \\
\text { La Micoque }\end{array}$} & \multirow{2}{*}{$\begin{array}{c}E \\
\text { mosbachensis } \\
\begin{array}{l}\text { Biache St Vaast } \\
\text { (Auguste 1995) }\end{array}\end{array}$} & \multirow{2}{*}{$\begin{array}{c}\begin{array}{c}E . \mathrm{cf} \\
\text { mosbachensis }\end{array} \\
\text { Achenheim } \\
\text { (Prat 1968) }\end{array}$} & \multirow{2}{*}{$\begin{array}{c}\text { E. mosb. } \\
\text { campdepeyni } \\
\text { Camp-de- } \\
\text { Peyre } \\
\text { (Guadelli et } \\
\text { Prat 1995) }\end{array}$} & \multirow{2}{*}{$\begin{array}{c}\begin{array}{c}\text { E. mosb. } \\
\text { tautavelensis }\end{array} \\
\begin{array}{c}\text { Caune de rArago } \\
\text { (Crégut 1979) }\end{array}\end{array}$} & \multicolumn{2}{|c|}{ E. caballus germanicus } & \multicolumn{3}{|c|}{ E. caballus gallicus } \\
\hline & & & & & & $\begin{array}{l}\text { Pair-non-Pair } \\
\text { (Prat 1968) }\end{array}$ & \begin{tabular}{|c} 
Combe-Grenal \\
(Prat 1968)
\end{tabular} & $\begin{array}{c}\text { Solutré } \\
\text { (Guadelli 1987) }\end{array}$ & $\begin{array}{c}\text { St Germain-la- } \\
\text { Rivere } \\
\text { (Prat 1968) }\end{array}$ & $\begin{array}{c}\text { Camiac } \\
\text { (Guadelili 1987) }\end{array}$ \\
\hline DAP prox. & $\begin{array}{c}\mathrm{n}=4 \\
34,4-37,6 \\
35,75+1-1,34 \\
1,37\end{array}$ & $\begin{array}{c}n=5 \\
33,7-39,9 \\
36,32+1-1,82^{*} \\
2,08\end{array}$ & $\begin{array}{c}n=4 \\
39,5-42,0 \\
40,625+1-1,09 \\
1,11\end{array}$ & $\begin{array}{c}n=6 \\
35,0.039,5 \\
37.8\end{array}$ & $\begin{array}{c}\mathrm{n}=8 \\
34,6-42,3 \\
39,15+l-1,54^{*} \\
2,22\end{array}$ & $\begin{array}{c}\mathrm{n}=24 \\
34,0.41,0 \\
37.5\end{array}$ & $33.5 ; 35.5$ & $\begin{array}{c}\mathrm{n}=53 \\
29,0-39,0 \\
34,71+1-0,50 \\
1,80\end{array}$ & $\begin{array}{c}\mathrm{n}=7 \\
33,0-36,5 \\
34,64+1-0,82^{*} \\
1,11\end{array}$ & $\begin{array}{c}\mathrm{n}=6 \\
34,4-38,1 \\
35,97+1-1,27 \\
1,21\end{array}$ \\
\hline DT maxi prox. & 58.6 & & $\begin{array}{c}n=5 \\
57,5-60,0 \\
58,8+1-1,10^{*} \\
1,25\end{array}$ & $\begin{array}{c}n=5 \\
52.0-56,5 \\
53,8\end{array}$ & $\begin{array}{c}\mathrm{n}=9 \\
53,2-67,9 \\
57,83+1-1,65^{*} \\
2,52\end{array}$ & $\begin{array}{c}n=24 \\
48,0.58,5 \\
54,5\end{array}$ & $54,5: 53,5$ & $\begin{array}{c}\mathrm{n}=61 \\
47,5-59,0 \\
52,71+1-0,60 \\
2,36\end{array}$ & $\begin{array}{c}\mathrm{n}=8 \\
49,0-52,5 \\
50,88+1,-9,90^{\circ} \\
1,30^{*}\end{array}$ & \\
\hline $\begin{array}{l}\text { DT articulaire } \\
\text { prox. }\end{array}$ & $\begin{array}{c}\mathrm{n}=3 \\
52,5-54,6 \\
53,9+1,1,37 \\
1,21\end{array}$ & $\begin{array}{c}\mathrm{n}=4 \\
53,2-61,0 \\
55,72+l-3,05^{*} \\
3,11\end{array}$ & $\begin{array}{c}n=3 \\
57,0-58,0 \\
57,67+1,-0,65 \\
0,58\end{array}$ & $\begin{array}{c}n=4 \\
50,5-56,5 \\
54,1\end{array}$ & $\begin{array}{c}n=9 \\
55,4-60,4 \\
57,05+1-1,21^{*} \\
1,86\end{array}$ & $\begin{array}{c}\mathrm{n}=24 \\
48,0-57,0\end{array}$ & 52,$0 ; 52,5$ & $\begin{array}{c}\mathrm{n}=52 \\
46,5-57,0 \\
51,37+1-0,62 \\
2,23\end{array}$ & 50,$0 ; 47,0$ & \\
\hline $\begin{array}{l}\text { DAP sus- } \\
\text { articulaire dist. }\end{array}$ & $\begin{array}{c}\mathrm{n}=7 \\
23,2-30,0 \\
26,57+1-1,84 \\
2,49\end{array}$ & & $\begin{array}{c}n=4 \\
25,0-27,0 \\
26,25+l-0,94^{*} \\
0,96\end{array}$ & $\begin{array}{c}n=5 \\
25,5.28 .0 \\
26,6\end{array}$ & $\begin{array}{c}\mathrm{n}=6 \\
24,3-29,0 \\
26,4+1-1,54^{*} \\
1,92\end{array}$ & $\begin{array}{c}n=24 \\
23,0.28,5 \\
25,4\end{array}$ & 26,0 & $\begin{array}{c}\mathrm{n}=35 \\
21,0-27,8 \\
23,76+1-0,47 \\
1,35\end{array}$ & $\begin{array}{c}n=43 \\
22,0-25,5 \\
23,5\end{array}$ & $\begin{array}{l}23,4 ; 26,0 ; \\
26,5\end{array}$ \\
\hline $\begin{array}{c}\text { DT sus-articulaire } \\
\text { dist. }\end{array}$ & $\begin{array}{c}\mathrm{n}=7 \\
50,8-54,3 \\
53,01+1-0,94 \\
1,27 \\
\end{array}$ & $\begin{array}{c}\mathrm{n}=6 \\
51,7-55,7 \\
53,22+l-1,14^{*} \\
1,43\end{array}$ & $\begin{array}{c}n=5 \\
52,0^{2}-58,5 \\
54,9+1,2,41^{*} \\
2,75\end{array}$ & $\begin{array}{c}n=6 \\
51,0-58,0 \\
54,4\end{array}$ & $\begin{array}{c}\mathrm{n}=4 \\
52,4-56,3 \\
54,05+1-1,61^{*} \\
1,64\end{array}$ & $\begin{array}{c}n=24 \\
49.0-54,0 \\
51,95\end{array}$ & 50,0 & $\begin{array}{c}\mathrm{n}=36 \\
45,0-54,3 \\
50,27+1-0,69 \\
2,02 \\
\end{array}$ & $\begin{array}{c}n=43 \\
43.5-53 \\
49,37\end{array}$ & $\begin{array}{c}49,7 ; 51,5 ; \\
50,8\end{array}$ \\
\hline $\begin{array}{l}\text { DT articulaire } \\
\text { dist. }\end{array}$ & $\begin{array}{c}\mathrm{n}=6 \\
52,3-54,0 \\
53,17+1-0,58 \\
0,73\end{array}$ & $\begin{array}{c}\mathrm{n}=4 \\
55,2-56,6 \\
55,07+1,1,28^{*} \\
1,31\end{array}$ & $\begin{array}{c}\mathrm{n}=5 \\
54,0^{-60,5} \\
56,7+l-2,37^{*} \\
2,71\end{array}$ & $\begin{array}{c}n=5 \\
51,0-56,0 \\
54,4\end{array}$ & $\begin{array}{c}\mathrm{n}=6 \\
54,0-58,0 \\
56,36+1,1,38^{*} \\
1,72\end{array}$ & $\begin{array}{c}n=24 \\
50,0 \cdot 56,0 \\
53,2\end{array}$ & 52,0 & $\begin{array}{c}\mathrm{n}=34 \\
46,0-56,5 \\
51,48+1-0,88 \\
2,52\end{array}$ & \begin{tabular}{c|}
$\mathrm{n}=43$ \\
$46,0-55,0$ \\
$50,88+1-0,64$ \\
2,07
\end{tabular} & $\begin{array}{c}49,7: 51,0: \\
51,0\end{array}$ \\
\hline
\end{tabular}

Tableau 23 - Dimension des première et deuxième phalanges antérieures du Cheval de La Micoque.

Table 23 - Measurements of first and second anterior phalanxes of the Horse of La Micoque.

\begin{tabular}{|c|c|c|}
\hline $\begin{array}{c}\text { Equus mosb. } \\
\text { micoquii }\end{array}$ & $\begin{array}{c}1 \text { ère phalange } \\
\text { antérieure }\end{array}$ & $\begin{array}{c}2^{\text {ème }} \text { phalange } \\
\text { antérieure }\end{array}$ \\
\hline Hauteur totale & 90,$5 ; 95,8$ & $\begin{array}{c}n=4 \\
43,5-52,5 \\
48,8+/-3735 \\
3,8\end{array}$ \\
\hline $\begin{array}{l}\text { Hauteur } \\
\text { antérieure }\end{array}$ & 79,$0 ; 86,5$ & $\begin{array}{c}\mathrm{n}=5 \\
36,4-39,9 \\
38,4+/-1,27 \\
1,45\end{array}$ \\
\hline $\begin{array}{c}\text { Hauteur } \\
\text { postérieure }\end{array}$ & 79,$0 ; 81,4$ & \\
\hline DAP proximal & 39,$0 ; 47,5$ & $\begin{array}{c}\mathrm{n}=3 \\
33,2-33,6 \\
33,33+/-0,26 \\
0,23\end{array}$ \\
\hline DT proximal & 65,$0 ; 67,0$ & $\begin{array}{c}\mathrm{n}=4 \\
57,0-61,2 \\
58,6+/-1,84 \\
1,87\end{array}$ \\
\hline DT milieu & 42,$3 ; 43,1$ & $\begin{array}{c}n=5 \\
47,6-52,4 \\
50,3+/-1,5 \\
1,74 \\
\end{array}$ \\
\hline $\begin{array}{l}\text { DT sus- } \\
\text { articulaire distal }\end{array}$ & 52,$2 ; 54,7$ & $\begin{array}{c}\mathrm{n}=5 \\
48,0-57,2 \\
53,8+/-3,03 \\
3,46\end{array}$ \\
\hline \begin{tabular}{|c} 
DT articulaire \\
distal
\end{tabular} & 50,$4 ; 45,7$ & \\
\hline
\end{tabular}


Tableau 24 - Fémur. Diamètre antéro-postérieur de la tête fémorale des Chevaux de La Micoque et de Caune de l'Arago.

Table 24 - Femur. Antero-posterior diameter of femoral head of Horses of La Micoque and Caune de l'Arago.

\begin{tabular}{|c|c|c|}
\cline { 2 - 3 } \multicolumn{1}{c|}{} & $\begin{array}{c}\text { E. mosb. } \\
\text { micoquii }\end{array}$ & $\begin{array}{c}\text { E. mosb. } \\
\text { tautavelensis }\end{array}$ \\
\cline { 2 - 3 } & La Micoque & $\begin{array}{c}\text { Caune de l'Arago } \\
\text { (Crégut 1979) }\end{array}$ \\
\hline DAP tête art. & $61,3-65,6$ & $\mathrm{n}=14$ \\
prox. & $63,95+/-1,91$ & $64,0-67,1$ \\
& 1,95 & 1,88 \\
\hline
\end{tabular}

Tableau 25 - Patella. Diamètre transversal et hauteur. Table 25 - Patella. Transversal diameter and height.

\begin{tabular}{|c|c|c|}
\cline { 2 - 3 } \multicolumn{1}{c|}{} & $\begin{array}{c}\text { E. mosb. } \\
\text { micoquii }\end{array}$ & $\begin{array}{c}\text { E. mosb. } \\
\text { tautavelensis }\end{array}$ \\
\cline { 2 - 3 } & La Micoque & $\begin{array}{c}\text { Caune de l'Arago } \\
\text { (Crégut 1979) }\end{array}$ \\
\hline & & $\mathrm{n}=10$ \\
DT & 73,1 & $\begin{array}{c}71,5-81,0 \\
76,58+/-1,33^{*} \\
2,15\end{array}$ \\
\hline & & $\mathrm{n}=10$ \\
Hauteur & 72,6 & $71,0-79,5$ \\
& & $76,4+/-1,46^{*}$ \\
& & 2,36 \\
\hline
\end{tabular}


Tableau 26 - Tibia. Diamètres antéro-postérieur et transversal de l'extrémité distale. Table 26 - Tibia. Antero-posterior and transversal diameters of distal extremity.

\begin{tabular}{|c|c|c|c|c|c|c|}
\hline \multirow[b]{2}{*}{ Tibia } & \multirow{2}{*}{$\begin{array}{l}\text { E. mosb. } \\
\text { micoquii } \\
\text { La Micoque }\end{array}$} & \multirow{2}{*}{$\begin{array}{c}\text { E. mosbachensis } \\
\text { Mosbach, } \\
\text { Reichenau } \\
\text { (in Prat 1968) }\end{array}$} & \multicolumn{2}{|c|}{ E. cf. mosbachensis } & \multirow{2}{*}{$\begin{array}{c}\text { E. mosb. } \\
\text { tautavelensis } \\
\begin{array}{c}\text { Caune de l'Arago } \\
\text { (Crégut 1979) }\end{array}\end{array}$} & \multirow{2}{*}{$\begin{array}{l}\begin{array}{c}\text { E. caballus } \\
\text { piveteaui }\end{array} \\
\text { Abri Suard } \\
\text { (Prat 1968) }\end{array}$} \\
\hline & & & $\begin{array}{l}\text { Montoussé } \\
\text { (Prat 1968) }\end{array}$ & $\begin{array}{l}\text { Achenheim } \\
\text { (Prat 1968) }\end{array}$ & & \\
\hline DT dist. & $\begin{array}{c}\mathrm{n}=6 \\
76,5-88,5 \quad 82,4 \\
+/-3,88 \\
4,85 \\
\end{array}$ & 88,$0 ; 91,0 ; 95,0$ & 92,$0 ; 94,0$ & 96 & $\begin{array}{c}\mathrm{n}=12 \\
80,6-89,5 \\
85,82+/-1,54^{*} \\
2,73 \\
\end{array}$ & $\begin{array}{c}\mathrm{n}=9 \\
80,5-89,0 \\
84,05+/-1,80^{*} \\
2,76 \\
\end{array}$ \\
\hline DAP dist. & $\begin{array}{c}\mathrm{n}=10 \\
47,5-54,5 \\
51,71+/-1,38 \\
2,23 \\
\end{array}$ & & 56,$0 ; 56,0$ & 57,5 & $\begin{array}{c}\mathrm{n}=12 \\
50,3-56,5 \\
53,13+/-1,10^{*} \\
1,94\end{array}$ & $\begin{array}{c}\mathrm{n}=9 \\
51,5-55,5 \\
52,94+/-0,96^{*} \\
1,47^{*}\end{array}$ \\
\hline
\end{tabular}

Tableau 27 - Dimensions des talus du Cheval de La Micoque, d'Equus mosbachensis et d'Equus caballus piveteaui.

Table 27 - Measurements of talus of the Horse of La Micoque, of Equus mosbachensis and Equus caballus piveteaui.

\begin{tabular}{|c|c|c|c|c|c|}
\hline \multirow{2}{*}{ Talus } & $\begin{array}{l}\text { E. mosb. } \\
\text { micoquii }\end{array}$ & $\begin{array}{c}\text { E. cf. } \\
\text { mosbachensis }\end{array}$ & $\begin{array}{l}\text { E. mosb. } \\
\text { palustris }\end{array}$ & $\begin{array}{c}\text { E. mosb. } \\
\text { tautavelensis }\end{array}$ & $\begin{array}{l}\text { E. caballus } \\
\text { piveteaui }\end{array}$ \\
\hline & La Micoque & $\begin{array}{l}\text { Achenheim } \\
\text { (Prat 1968) }\end{array}$ & $\begin{array}{c}\text { Lunel-Viel } \\
\text { (Bonifay 1980) }\end{array}$ & $\begin{array}{c}\text { Caune de l'Arago } \\
\text { (Crégut 1979) }\end{array}$ & $\begin{array}{l}\text { Abri Suard } \\
\text { (Prat 1968) }\end{array}$ \\
\hline $\begin{array}{l}\text { Hauteur } \\
\text { totale }\end{array}$ & $\begin{array}{c}\mathrm{n}=9 \\
62,9-69,6 \\
65,79+/-1,31 \\
2,01 \\
\end{array}$ & $\begin{array}{c}\mathrm{n}=12 \\
63,5-76,0 \\
67,62+/-2,24 \\
3,57 \\
\end{array}$ & $\begin{array}{c}\mathrm{n}=24 \\
63,2-68,0 \\
64,50+/-0,48^{*} \\
1,20 \\
\end{array}$ & $\begin{array}{c}\mathrm{n}=18 \\
61,8-70,5 \\
66,18+/-1,12 \\
2,26 \\
\end{array}$ & $\begin{array}{c}n=5 \\
66,0-69,5 \\
68,0\end{array}$ \\
\hline DT maxi & $\begin{array}{c}\mathrm{n}=9 \\
64,8-71,1 \\
68,31+/-1,43 \\
2,19\end{array}$ & & $\begin{array}{c}\mathrm{n}=24 \\
63,3-72,8 \\
69,07+/-0,90^{*} \\
2,26\end{array}$ & & \\
\hline $\begin{array}{c}\text { DT de la lèvre } \\
\text { interne à la } \\
\text { trochlée }\end{array}$ & $\begin{array}{c}\mathrm{n}=11 \\
57,7-65,6 \\
61,84+/-1,59 \\
2,43 \\
\end{array}$ & $\begin{array}{c}\mathrm{n}=12 \\
68,0-76,0 \\
71,6+/-2,25 \\
3,19 \\
\end{array}$ & $\begin{array}{c}\mathrm{n}=24 \\
63,9-71,8 \\
66,63+/-0,85^{\star} \\
2,13 \\
\end{array}$ & $\begin{array}{c}\mathrm{n}=18 \\
65,3-73,1 \\
68,74+/-1,08 \\
2,18 \\
\end{array}$ & $\begin{array}{c}n=5 \\
66,5-72,5 \\
69,8 \\
\end{array}$ \\
\hline DAP médial & $\begin{array}{c}\mathrm{n}=7 \\
41,5-44,1 \\
42,87+/-0,68 \\
1,05\end{array}$ & & & & \\
\hline $\begin{array}{c}\text { DT distal de } \\
\text { la trochlée }\end{array}$ & $\begin{array}{c}\mathrm{n}=9 \\
45,2-51,5 \\
47,94+/-1,13 \\
1,73\end{array}$ & $\begin{array}{c}n=12 \\
46,0-55,0 \\
49,6\end{array}$ & & $\begin{array}{c}\mathrm{n}=17 \\
46,0-53,9 \\
49,13+/-1,00 \\
1,95\end{array}$ & $\begin{array}{l}n=5 \\
49,0\end{array}$ \\
\hline $\begin{array}{c}\text { DT articulaire } \\
\text { distal }\end{array}$ & $\begin{array}{c}n=7 \\
57,3-62,0 \\
58,69+/-1,05 \\
1,61 \\
\end{array}$ & $\begin{array}{c}n=12 \\
56,5-66,0 \\
61,1\end{array}$ & $\begin{array}{c}\mathrm{n}=24 \\
50,5-61,0 \\
56,23+/-0,98^{*} \\
2,45 \\
\end{array}$ & $\begin{array}{c}\mathrm{n}=16 \\
54,4-61,5 \\
58,56+/-1,06 \\
1,93 \\
\end{array}$ & $\begin{array}{c}n=4 \\
57,5-62,0 \\
60,0\end{array}$ \\
\hline $\begin{array}{c}\text { DAP } \\
\text { articulaire } \\
\text { distal } \\
\end{array}$ & $\begin{array}{c}n=8 \\
36,3-42,2 \\
39,36+/-1,10 \\
1,68 \\
\end{array}$ & $\begin{array}{c}n=12 \\
37,0-42,5\end{array}$ & $\begin{array}{c}\mathrm{n}=23 \\
36,8-42,0 \\
39,27+/-0,66^{*} \\
1,62\end{array}$ & $\begin{array}{c}\mathrm{n}=17 \\
36,2-42,4 \\
39,16+/-0,87 \\
1,71 \\
\end{array}$ & $\begin{array}{c}n=4 \\
39,5-41,0 \\
40,0\end{array}$ \\
\hline
\end{tabular}


Tableau 28 - Dimensions des calcaneus des Chevaux de La Micoque et de Lunel-Viel et d'Equus caballus.

Table 28 - Measurements of calcaneus of Horses of La Micoque and Lunel-Viel and of Equus caballus.

\begin{tabular}{|c|c|c|c|c|c|}
\hline \multirow{2}{*}{ Calcaneus } & $\begin{array}{l}\text { E. mosb. } \\
\text { micoquii }\end{array}$ & E. mosb. palustris & $\begin{array}{l}\text { E. cab.cf. } \\
\text { germanicus }\end{array}$ & $\begin{array}{l}\text { E. caballus } \\
\text { gallicus }\end{array}$ & $\begin{array}{l}\text { E. caballus } \\
\text { przewalskii }\end{array}$ \\
\hline & La Micoque & $\begin{array}{c}\text { Lunel-Viel } \\
\text { (Bonifay 1980) }\end{array}$ & $\begin{array}{l}\text { Pair-non-Pair } \\
\text { (Prat 1968) }\end{array}$ & $\begin{array}{c}\text { Solutré } \\
\text { (Guadelli1987) }\end{array}$ & $\begin{array}{c}\text { (Gromova } \\
1949 \text { b) }\end{array}$ \\
\hline $\begin{array}{l}\text { Longueur } \\
\text { totale }\end{array}$ & $\begin{array}{c}\mathrm{n}=3 \\
100,8-109,7 \\
106,17+/-5,35 \\
4,72\end{array}$ & $\begin{array}{c}\mathrm{n}=11 \\
113,9-122,0 \\
117,87+/-2,16^{*} \\
3,66\end{array}$ & $\begin{array}{c}n=15 \\
110,5-121,0 \\
115,33+/-2,29 \\
3,62 \\
\end{array}$ & $\begin{array}{c}\mathrm{n}=19 \\
105,0-116,0 \\
110,61+/-1,35 \\
2,80\end{array}$ & $\begin{array}{c}99,0-111,5 \\
104,8\end{array}$ \\
\hline $\begin{array}{c}\text { Longueur de } \\
\text { la partie } \\
\text { proximale }\end{array}$ & $\begin{array}{c}\mathrm{n}=4 \\
67,5-87,0 \\
77,35+/-12,35 \\
10,92 \\
\end{array}$ & & & $\begin{array}{c}\mathrm{n}=12 \\
75,0-83,0 \\
79,23+/-1,58 \\
2,48 \\
\end{array}$ & \\
\hline $\begin{array}{c}\text { DT maxi de la } \\
\text { tubérosité } \\
\text { proximale }\end{array}$ & $\begin{array}{c}n=3 \\
34,7-37,6 \\
36,43+/-1,73 \\
1,53\end{array}$ & $\begin{array}{c}\mathrm{n}=9 \\
30,5-38,5 \\
34,97+/-1,80^{*} \\
2,76\end{array}$ & $\begin{array}{c}n=15 \\
33,0-39,0\end{array}$ & $\begin{array}{c}\mathrm{n}=17 \\
30,0-37,5 \\
34,19+/-0,99 \\
1,92\end{array}$ & \\
\hline $\begin{array}{c}\text { DAP maxi de } \\
\text { la tubérosité } \\
\text { proximale }\end{array}$ & $\begin{array}{c}n=2 \\
58,0 ; 59,3 \\
58,65\end{array}$ & $\begin{array}{c}\mathrm{n}=8 \\
55,3-59,7 \\
57,41+/-1,12^{*} \\
1,62\end{array}$ & $\begin{array}{c}n=15 \\
49,5-62,0 \\
55,0\end{array}$ & $\begin{array}{c}\mathrm{n}=20 \\
46,0-55,0 \\
51,72+/-1,09 \\
2,34\end{array}$ & \\
\hline $\begin{array}{c}\text { DT mini de la } \\
\text { tubérosité } \\
\text { proximale }\end{array}$ & $\begin{array}{c}\mathrm{n}=6 \\
21,1-25,2 \\
23,32+/-2,23 \\
1,97\end{array}$ & & $\begin{array}{c}n=15 \\
20,5-24,0 \\
22,3\end{array}$ & $\begin{array}{c}\mathrm{n}=14 \\
19,0-23,6 \\
21,46+/-0,85 \\
1,47\end{array}$ & \\
\hline $\begin{array}{c}\text { DAP mini de } \\
\text { la tubérosité } \\
\text { proximale }\end{array}$ & $\begin{array}{c}\mathrm{n}=5 \\
42,9-53,0 \\
47,6+/-4,95 \\
4,38\end{array}$ & & $\begin{array}{c}n=15 \\
42,5-52,0 \\
46,4\end{array}$ & $\begin{array}{c}\mathrm{n}=20 \\
41,0-47,5 \\
44,36+/-0,77 \\
1,66 \\
\end{array}$ & \\
\hline DT maxi & $\begin{array}{c}\mathrm{n}=2 \\
51,6-57,8 \\
54,7\end{array}$ & $\begin{array}{c}\mathrm{n}=11 \\
49,5-60,6 \\
55,76+/-1,84^{*} \\
3,11\end{array}$ & & $\begin{array}{c}\mathrm{n}=13 \\
49,0-58,0 \\
53,65+/-1,59 \\
2,62\end{array}$ & \\
\hline $\begin{array}{c}\text { DAP au } \\
\text { niveau du bec }\end{array}$ & $\begin{array}{c}n=2 \\
51,2 ; 54,5 \\
52,85\end{array}$ & & $\begin{array}{c}n=15 \\
48,0-57,5 \\
53,8\end{array}$ & $\begin{array}{c}n=18 \\
47,5-55,5 \\
51,5+/-1,25 \\
2,51\end{array}$ & \\
\hline
\end{tabular}

Tableau 29 - Dimensions des naviculaires et cuboïdes du Cheval de La Micoque, d'Equus mosbachensis et d'Equus caballus.

Table 29 - Measurements of naviculaires and cuboides of the Horse of La Micoque, of Equus mosbachensis and Equus caballus.

\begin{tabular}{|c|c|c|c|c|c|c|c|c|}
\hline & & $\begin{array}{l}\text { E. mosb. } \\
\text { micoquii }\end{array}$ & $\begin{array}{c}\text { E. mosb. } \\
\text { tautavelensis }\end{array}$ & $\begin{array}{l}\text { E. mosb. } \\
\text { palustris }\end{array}$ & $\begin{array}{c}\text { E. caballus } \\
\text { piveteaui }\end{array}$ & $\begin{array}{l}\text { E. caballus } \\
\text { germanicus }\end{array}$ & \multicolumn{2}{|c|}{ E. caballus gallicus } \\
\hline & & La Micoque & $\begin{array}{c}\text { Caune de } \\
\text { l'Arago } \\
\text { (Crégut 1979) }\end{array}$ & $\begin{array}{c}\text { Lunel-Viel } \\
\text { (Bonifay 1980) }\end{array}$ & $\begin{array}{l}\text { Abri Suard } \\
\text { (Prat 1968) }\end{array}$ & $\begin{array}{l}\text { Pair-non-Pair } \\
\text { (Prat 1968) }\end{array}$ & $\begin{array}{c}\text { Solutré } \\
\text { (Guadelli 1987) }\end{array}$ & $\begin{array}{c}\text { St-Germain-la- } \\
\text { Rivière } \\
\text { (Prat 1968) }\end{array}$ \\
\hline \multirow{2}{*}{$\begin{array}{l}\frac{0}{\frac{2}{\pi}} \\
\frac{\pi}{3} \\
\frac{0}{3} \\
\frac{\pi}{2}\end{array}$} & DAP & $\begin{array}{c}n=12 \\
42,3-52,3 \\
48,37+/-1,45 \\
2,57\end{array}$ & $\begin{array}{c}\mathrm{n}=20 \\
44,0-54,0 \\
49,06+/-1,30 \\
2,78 \\
\end{array}$ & $\begin{array}{c}\mathrm{n}=4 \\
45,5-48,2 \\
46,88+/-1,11^{\star} \\
1,13^{\star} \\
\end{array}$ & $\begin{array}{c}\mathrm{n}=6 \\
45,0-50,5 \\
46,67+/-1,57^{\star} \\
1,97^{\star}\end{array}$ & $\begin{array}{c}n=16 \\
41,5-50,0 \\
46,5\end{array}$ & 43,$5 ; 45,0$ & $\begin{array}{c}\mathrm{n}=6 \\
41,5-48,0 \\
44,33+/-2,27^{\star} \\
2,84^{*}\end{array}$ \\
\hline & DT & $\begin{array}{c}\mathrm{n}=12 \\
53,6-60,6 \\
58,19+/-1,23 \\
2,17 \\
\end{array}$ & $\begin{array}{c}n=20 \\
56,4-64,8 \\
59,33+/-1,46 \\
3,14 \\
\end{array}$ & $\begin{array}{c}\mathrm{n}=4 \\
56,0-61,0 \\
58,50+/-2,15^{\star} \\
2,20^{\star} \\
\end{array}$ & $\begin{array}{c}\mathrm{n}=7 \\
56,0-62,5 \\
59,14+/-1,80^{\star} \\
2,43^{*} \\
\end{array}$ & $\begin{array}{c}n=16 \\
52,5-60,0 \\
55,8\end{array}$ & 52,$4 ; 53,5$ & $\begin{array}{c}\mathrm{n}=6 \\
50,5-57,5 \\
54,25+/-2,17^{\star} \\
2,72^{*}\end{array}$ \\
\hline \multirow{2}{*}{$\begin{array}{l}\frac{0}{0} \\
: 0 \\
0\end{array}$} & DAP & $\begin{array}{c}\mathrm{n}=15 \\
38,3-44,7 \\
41,77+/-0,84 \\
1,72\end{array}$ & $\begin{array}{c}n=28 \\
39,6-45,2 \\
42,33+/-0,55 \\
1,43\end{array}$ & $\begin{array}{c}n=3 \\
41,0-42,8 \\
42,1+/-1,09^{\star} \\
0,96^{*}\end{array}$ & & $\begin{array}{c}n=12 \\
38,0-43,0 \\
40,85\end{array}$ & $\begin{array}{c}n=28 \\
33,5-41,5 \\
38,36+/-0,78 \\
2,00 \\
\end{array}$ & \\
\hline & DT & $\begin{array}{c}\mathrm{n}=15 \\
18,9-28,2 \\
23,79+/-1,35 \\
2,68\end{array}$ & $\begin{array}{c}n=26 \\
24,3-30,2 \\
26,85+/-0,59 \\
1,46\end{array}$ & $\begin{array}{c}\mathrm{n}=3 \\
26,0-26,5 \\
26,27+/-0,28^{\star} \\
0,25^{*}\end{array}$ & & $\begin{array}{c}n=12 \\
22,5-27,0 \\
25,0\end{array}$ & $\begin{array}{c}n=29 \\
20,0-27,5 \\
23,43+/-0,68 \\
1,78\end{array}$ & \\
\hline
\end{tabular}


Tableau 30 - Dimensions des cunéiformes du Cheval de La Micoque et de différents Équidés du Pléistocène.

Table 30 - Measurements of cuneiformes of the Horse of La Micoque and various Equids from Pleistocene.

\begin{tabular}{|c|c|c|c|c|c|c|c|c|}
\hline & \multicolumn{3}{|c|}{ Grand cunéiforme } & & \multicolumn{4}{|c|}{ Petit cunéiforme } \\
\hline & \multirow{2}{*}{$\begin{array}{l}\text { E. mosb. } \\
\text { micoquii } \\
\text { La Micoque }\end{array}$} & \multirow{2}{*}{$\begin{array}{c}\begin{array}{c}\text { E. mosb. } \\
\text { palustris }\end{array} \\
\begin{array}{c}\text { Lunel-Viel } \\
\text { (Bonifay 1980) }\end{array}\end{array}$} & \multirow{2}{*}{$\begin{array}{c}\begin{array}{c}\text { E. caballus } \\
\text { piveteaui }\end{array} \\
\text { Abri Suard } \\
\text { (Prat 1968) }\end{array}$} & & \multirow{2}{*}{$\begin{array}{l}\text { E. mosb. } \\
\text { micoquii } \\
\text { La Micoque }\end{array}$} & \multirow{2}{*}{$\begin{array}{c}\begin{array}{c}\text { E. mosb. } \\
\text { tautavelensis }\end{array} \\
\begin{array}{c}\text { Caune de } \\
\text { l'Arago } \\
\text { (Crégut 1979) }\end{array}\end{array}$} & \multicolumn{2}{|c|}{ E. caballus gallicus } \\
\hline & & & & & & & \begin{tabular}{|c|} 
Camiac \\
(Guadelli 1987)
\end{tabular} & $\begin{array}{c}\text { Solutré } \\
\text { (Guadelli 1987) }\end{array}$ \\
\hline DAP & $\begin{array}{c}\mathrm{n}=7 \\
47,9-50,3 \\
48,74+1-0,60 \\
0,81 \\
\end{array}$ & 45,$0 ; 47,0$ & $\begin{array}{c}n=10 \\
48,0-52,0 \\
49,05+/-1,34^{\star} \\
2,17^{\star} \\
\end{array}$ & Longueur & $\begin{array}{c}\mathrm{n}=4 \\
33,9-40,1 \\
37,52+/-2,71 \\
2,77 \\
\end{array}$ & $\begin{array}{c}\mathrm{n}=12 \\
38,0-44,5 \\
40,98+/-1,08 \\
1,73\end{array}$ & 37,5 & $\begin{array}{c}\mathrm{n}=3 \\
34,0-37,4 \\
35,87+1-1,95^{*} \\
1,72^{\star}\end{array}$ \\
\hline DT & $\begin{array}{c}n=12 \\
51,6-57,7 \\
53,96+l-2,41 \\
4,25 \\
\end{array}$ & 51,$5 ; 56,2$ & $\begin{array}{c}\mathrm{n}=12 \\
52,5-61,0 \\
56,1+/-1,39^{\star} \\
2,46^{\star} \\
\end{array}$ & DT & $\begin{array}{c}\mathrm{n}=7 \\
14,9-18,6 \\
17,02+/-1,06 \\
1,43 \\
\end{array}$ & $\begin{array}{c}\mathrm{n}=13 \\
15,4-20,7 \\
17,98+/-0,95 \\
1,51\end{array}$ & 12,$4 ; 12,4$ & $\begin{array}{c}\mathrm{n}=3 \\
17,0-20,0 \\
19,00+/-1,96^{\star} \\
1,73^{\star}\end{array}$ \\
\hline & & & & Hauteur & 21 & $\begin{array}{c}\mathrm{n}=14 \\
21,0-24,5 \\
22,82+1-0,65 \\
1,09\end{array}$ & 19,9 & $\begin{array}{c}\mathrm{n}=3 \\
19,0-22,0 \\
20,33+1-1,73^{\star} \\
1,53^{\star}\end{array}$ \\
\hline
\end{tabular}

Tableau 31 - Dimensions des extrémités proximales des métatarsiens accessoires du Cheval de La Micoque.

Table 31 - Measurements of proximal extremities of secondary metatarsals of the Horse of La Micoque.

\begin{tabular}{|c|c|c|}
\cline { 2 - 3 } \multicolumn{1}{c|}{} & \multicolumn{2}{c|}{ E. mosbachensis micoquii } \\
\cline { 2 - 3 } \multicolumn{1}{c|}{} & Métatarsien II & Métatarsien IV \\
\hline \multirow{4}{*}{ DAP maxi } & $\mathrm{n}=18$ & $\mathrm{n}=28$ \\
& $18,9-26,3$ & $19,0-34,6$ \\
& $22,95+/-0,92$ & $29,96+/-0,94$ \\
& 1,99 & 1,25 \\
\hline & $\mathrm{n}=18$ & $\mathrm{n}=29$ \\
DT maxi & $11,7-22,2$ & $14,1-24,6$ \\
& $14,14+/-1,07$ & $20,42+/-0,62$ \\
& 2,31 & 0,82 \\
\hline
\end{tabular}

Tableau 32 - Dimensions des métatarsiens III du Cheval de La Micoque et des différents Équidés du Pléistocène.

Table 32 - Measurements of metatarsals III of the Horse of La Micoque and of various Equids from Pleistocene.

\begin{tabular}{|c|c|c|c|c|c|c|c|c|c|c|c|}
\hline \multirow[b]{2}{*}{ Metatarsien III } & $\begin{array}{l}\text { Em mosb- } \\
\text { micooguii }\end{array}$ & E. mosbachensis & $\underset{\substack{E \\
\text { mosbachensis }}}{\text { cf }}$ & 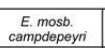 & $\begin{array}{c}\text { E, mosh. } \\
\text { tautavelensis }\end{array}$ & $\begin{array}{l}\begin{array}{l}\text { E. mossb } \\
\text { palustris }\end{array} \\
\text { int }\end{array}$ & 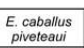 & 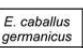 & \multicolumn{3}{|c|}{ E. caballus gallicus } \\
\hline & La Micoque & 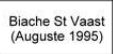 & $\begin{array}{l}\text { Achenheim } \\
\text { (Prat 1968) }\end{array}$ & 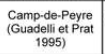 & $\begin{array}{l}\text { Caune de AArago } \\
\text { (Crígut 1979) }\end{array}$ & $\begin{array}{c}\text { Lunet-Vie } \\
\text { (Bonitay 1980) }\end{array}$ & $\begin{array}{l}\text { Abirisuard } \\
\text { (Prat 1988) }\end{array}$ & $\begin{array}{l}\text { Paitin-non parit } \\
\text { (Prat 1968) }\end{array}$ & $\begin{array}{c}\text { Camiac } \\
\text { (Guadelili 1987) }\end{array}$ & $\begin{array}{c}\text { Solutre } \\
\text { (Guadelli ig87) }\end{array}$ & 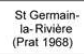 \\
\hline $\begin{array}{l}\text { D facette } \\
\text { cuboride }\end{array}$ & $\begin{array}{c}n=3 \\
11,2=12,4 \\
11,9+1.0 .71 \\
0.62\end{array}$ & 11,$9 ; 12,1$ & & $\begin{array}{l}12,7=7.75 .5 \\
14,0+1-0,7\end{array}$ & & & & & 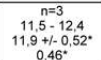 & 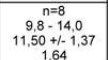 & \\
\hline $\begin{array}{c}\text { DT dist. uss- } \\
\text { articulaire- }\end{array}$ & 54,$5 ; 55,1$ & 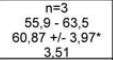 & $\begin{array}{c}n=10 \\
53.5 .60 .0 \\
56,95 \\
565\end{array}$ & $\begin{array}{c}n=8 \\
53.0 \\
55,1+5,0.1,3\end{array}$ & 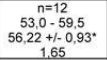 & 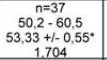 & $\begin{array}{c}n=4 \\
56,0.5,0.0 \\
57,6\end{array}$ & $\begin{array}{c}n=25 \\
46.55,5,0 \\
52.5\end{array}$ & $\begin{array}{c}50,5=5 \\
54,5 \\
52,40+1,1,37 \\
148\end{array}$ & 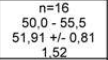 & $\begin{array}{c}n=43 \\
46,5.54,5 \\
500,7\end{array}$ \\
\hline $\begin{array}{l}\text { art dist } \\
\text { articulare }\end{array}$ & 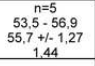 & 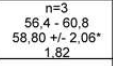 & $\begin{array}{c}n=7 \\
57,7=6,0.0 \\
58,36\end{array}$ & $\begin{array}{c}n=7 \\
53.57 .5 \\
55.7+1-1,1\end{array}$ & 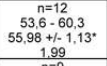 & 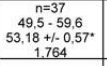 & $\begin{array}{c}n=5 \\
56.0 .0 .0 \\
58,5\end{array}$ & 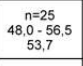 & 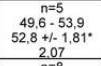 & 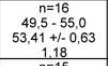 & 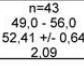 \\
\hline $\begin{array}{c}\text { DAP dist sus- } \\
\text { articulare }\end{array}$ & $28,4: 26,5$ & & $32.0 ; 31.0 ; 31.5$ & & 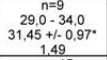 & & $\begin{array}{c}n=4 \\
27.54 .040 \\
30,4\end{array}$ & $\begin{array}{c}n=25 \\
28.0-3,5 \\
29,6 \\
29.6\end{array}$ & $\begin{array}{c}26,7=30.4 \\
28,71+10.96 \\
21.15\end{array}$ & 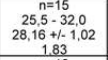 & $\begin{array}{c}n=43 \\
24,0.30,0 \\
27,3\end{array}$ \\
\hline $\begin{array}{l}\text { DAP Pist } \\
\text { aticulare }\end{array}$ & 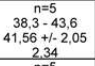 & 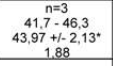 & 41,$5 ; 46,0 ; 43,0$ & 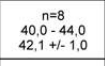 & 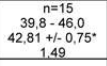 & 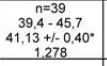 & $\begin{array}{c}n=6 \\
41.0 .46,0 \\
43,4 .\end{array}$ & $\begin{array}{l}n=25,0-44,0 \\
38\end{array}$ & & 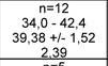 & $\begin{array}{c}n=43 \\
355.42 .0 \\
39.27 \\
\end{array}$ \\
\hline $\begin{array}{l}\text { DAP maxi } \\
\text { condyle interne }\end{array}$ & $\begin{array}{c}3=5.5 \\
=36,8 \\
35,42+1<-1.25 \\
1.42\end{array}$ & & & $\begin{array}{c}n=8 \\
31,7-36,7 \\
35,0+1-1,2\end{array}$ & & & & & & 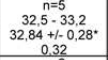 & \\
\hline $\begin{array}{c}\text { DAP mini } \\
\text { condyle interne }\end{array}$ & 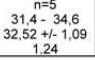 & 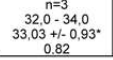 & & $\begin{array}{c}n=8 \\
30.0-33,2 \\
31,8+1-0,8\end{array}$ & & & & & & $\begin{array}{c}29,7=6 \\
30.8 \\
30,05 \\
30,0.041 \\
0.39\end{array}$ & \\
\hline
\end{tabular}


Tableau 33 - Dimensions des phalanges postérieures du Cheval de La Micoque et d'Equus mosbachensis.

Table 33 - Measurements of posterior phalanges of the Horse of La Micoque and Equus mosbachensis.

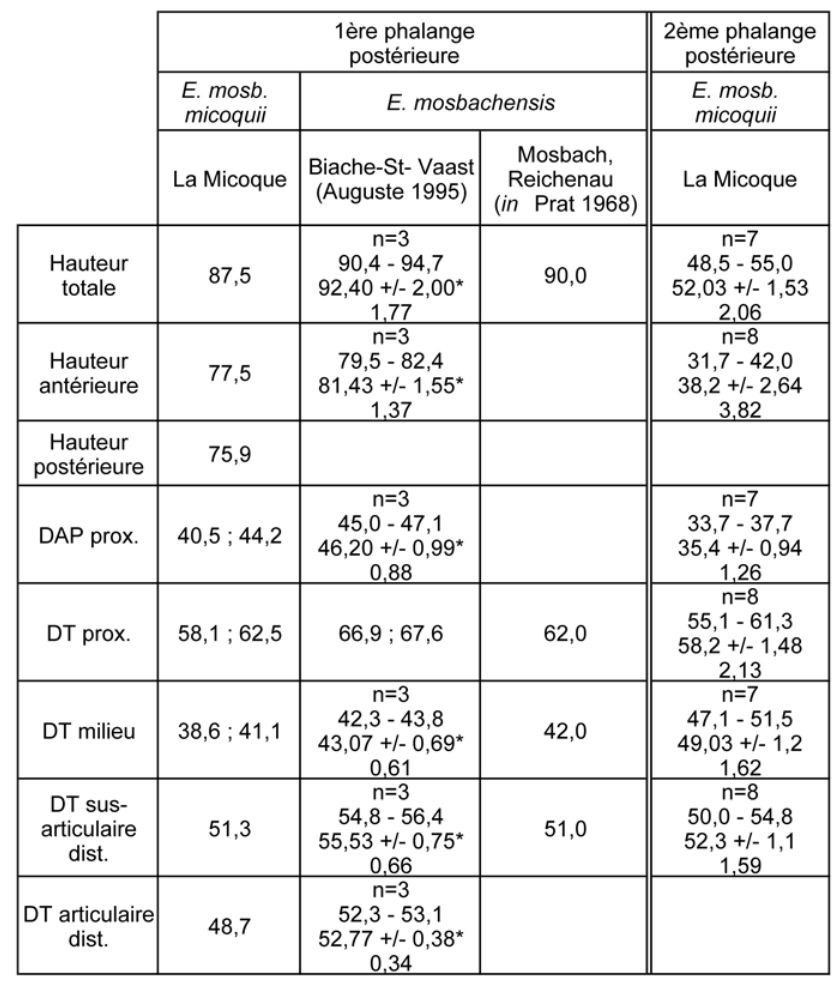

Tableau 34 - Succession des différentes espèces de chevaux de type caballin pléistocènes. Table 34 - Lineage of different Equids species of caballin type during the Pleistocene.

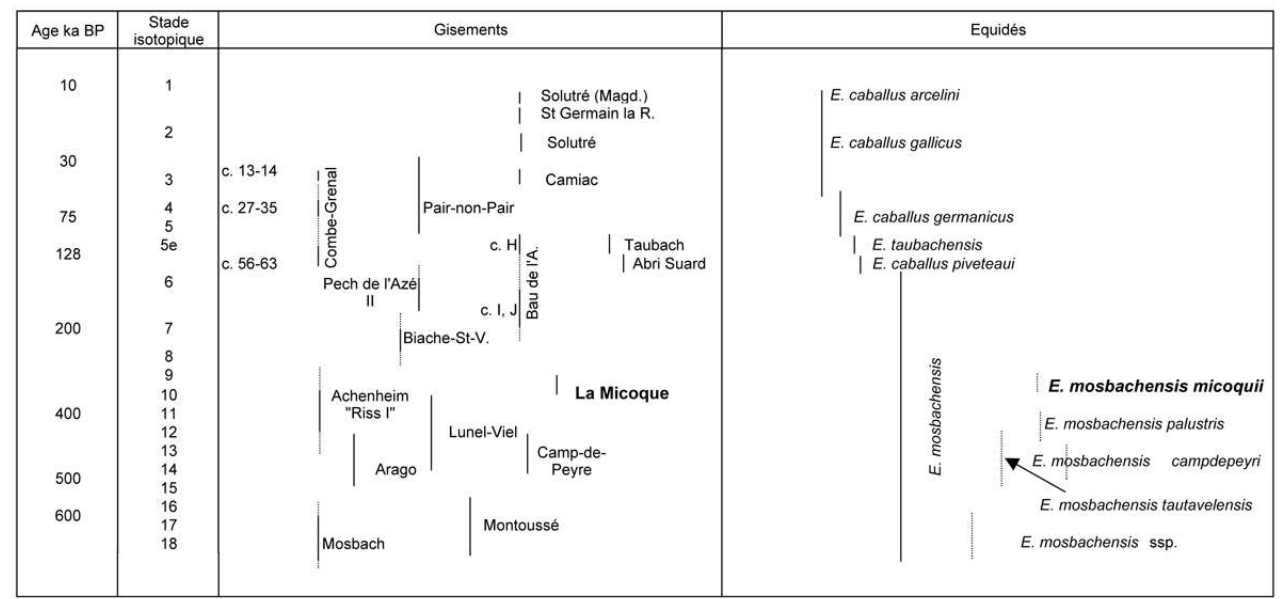

\section{NOTES}

1. Pour chacune des variables contenues dans les tableaux sont indiqués : le nombre de restes, l'intervalle de variation, la moyenne avec son intervalle de confiance à $95 \%$ et l'écart type. Les * correspondent à des valeurs calculées par nous même à partir des données publiées. 
2. En ce qui concerne les chevaux de Lunel-Viel et de Caune de l'Arago et compte tenu des caractéristiques morphologiques et biométriques des éléments du squelette, nous pensons que ces chevaux font partie du groupe des grands chevaux de type mosbachensis.

\section{RÉSUMÉS}

Le gisement de La Micoque (Les Eyzies-de-Tayac, Dordogne), dès sa découverte à la fin du XIX siècle, a intéressé de très nombreux fouilleurs et spécialistes. Il présente une longue stratigraphie qui a livré plusieurs assemblages lithiques et osseux originaux. Cependant, de nombreux points restaient encore à préciser comme le degré d'évolution du Cheval (taxon dominant) et la biostratigraphie $\mathrm{du}$ gisement. L'étude paléontologique a permis de mettre en évidence la présence d'un Cheval de l'espèce Equus mosbachensis présentant des caractères particuliers justifiant la création d'une sous-espèce: Equus mosbachensis micoquii et de positionner chronologiquement le site de La Micoque entre les sites de Lunel-Viel (Hérault) et Biache-SaintVaast (Pas-de-Calais).

Since its discovery in the late 19 th century, many archaeologists have been concerned by the site of "La Micoque" (Les Eyzies-de-Tayac, Dordogne). As a matter of fact this site shows a long stratigraphy which provided several lithic assemblages and many bone remains, presenting particular characteristics. However various points had to be specified such as the degree of evolution of the Horse (main taxon) and the biostratigraphical position of this site. Our palaeontological study allowed us to ascertain the existence of a horse belonging to the species Equus mosbachensis that justify the creation of a particular subspecies: Equus mosbachensis micoquii and allowed us to determine this site - from a chronological point of view - between the sites of Lunel-Viel (Hérault) and Biache-Saint-Vaast (Pas-de-Calais).

\section{INDEX}

Keywords : biostratigraphy, Equids, Equus mosbachensis micoquii, La Micoque, middle Pleistocene, palaeontology

Mots-clés : biostratigraphie, Equidés, Equus mosbachensis micoquii, La Micoque, paléontologie, Pléistocène moyen

\section{AUTEUR}

\section{ANNE LANGLOIS}

Université Bordeaux I, PACEA, UMR 5199, IPGQ, Bâtiment de Géologie (B18), Avenue des Facultés, 33405 Talence Cedex 